\title{
Synthesis and Transport Studies of a Cofacial Porphyrin Cyclophane
}

\author{
Patrick Zwick, ${ }^{a}{ }^{\text {} \ddagger}$ Chunwei Hsu $,{ }^{b}, \ddagger$ Maria El Abbassi, ${ }^{b}$ Olaf Fuhr,,${ }^{d, f}$ Dieter \\ Fenske, ${ }^{\mathrm{d}, \mathrm{f}}$ Diana Dulić, ${ }^{c}$ Herre S. J. van der Zant, ${ }^{*, b}$ and Marcel Mayor ${ }^{\star}, a, d, e$
}

\section{SUPPORTING INFORMATION}

\author{
*To whom correspondence should be addressed \\ ‡These authors contributed equally to this work
}

aDepartment of Chemistry, University of Basel, St. Johanns-Ring 19, 4056 Basel, Switzerland

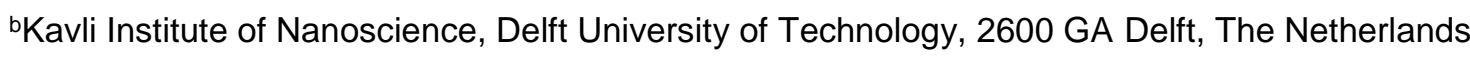

'Department of Physics and Department of Electrical Engineering, Faculty of Physical

and Mathematical Sciences, University of Chile, Avenida Blanco Encalada 2008, Santiago

$$
\text { 8330015, Chile. }
$$

dInstitute for Nanotechnology (INT), Karlsruhe Institute of Technology (KIT), P. O. Box 3640, 76021

Karlsruhe, Germany

eLehn Institute of Functional Materials (LIFM), School of Chemistry, Sun Yat-Sen University (SYSU), 510275 Guangzhou, China

fKarlsruhe Nano Micro Facility (KNMF), Karlsruhe Institute of Technology (KIT), P. O. Box 3640, 76021 Karlsruhe, Germany 


\section{TABLE OF FIGURES}

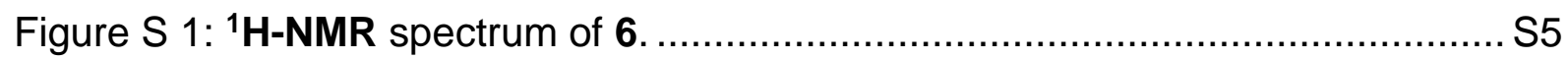

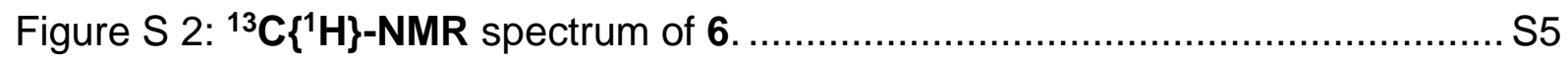

Figure $\mathrm{S} 3$ : Absorption spectrum of 6 measured in $\mathrm{CH}_{2} \mathrm{Cl}_{2} \ldots \ldots \ldots \ldots \ldots \ldots \ldots \ldots . . . . . . \ldots \ldots$

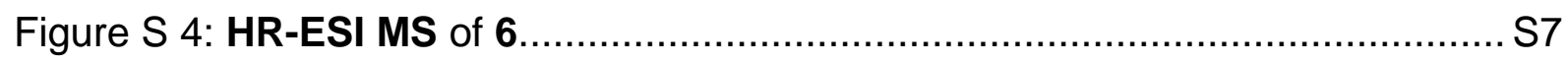

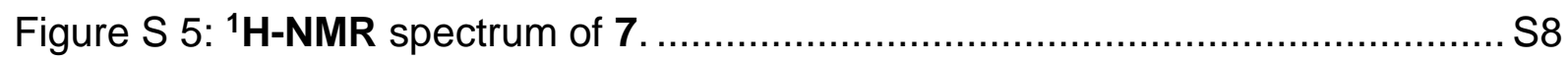

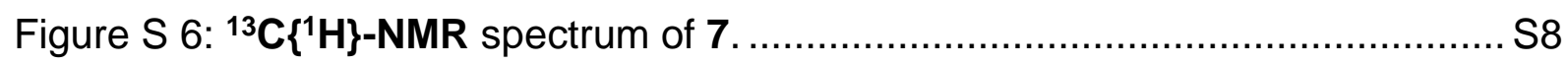

Figure S 7: Absorption spectrum of 7 measured in $\mathrm{CH}_{2} \mathrm{Cl}_{2} \ldots \ldots \ldots \ldots \ldots \ldots \ldots \ldots . . . \ldots . \ldots \ldots$

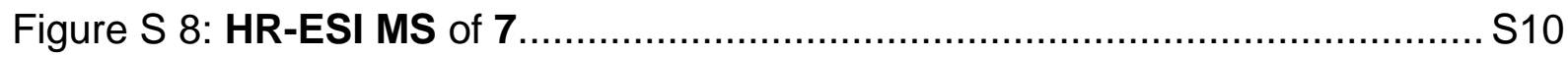

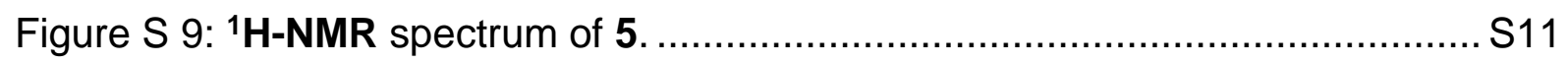

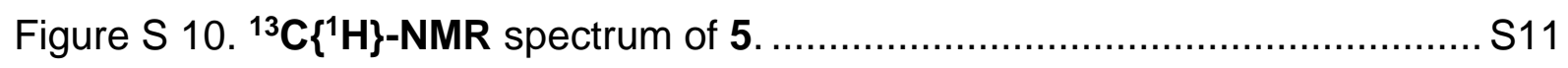

Figure $\mathrm{S}$ 11: Absorption spectrum of 5 measured in $\mathrm{CH}_{2} \mathrm{Cl}_{2} \ldots \ldots \ldots \ldots \ldots \ldots \ldots \ldots . . . . . . . . . . . .2$

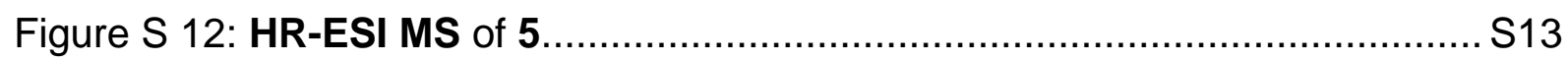

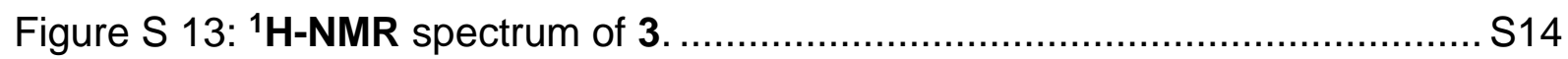

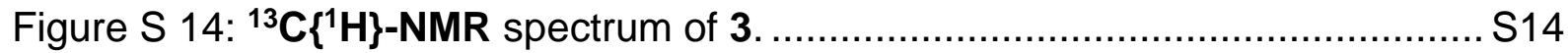

Figure S 15: Absorption spectra of a dilution series of 3 measured in $\mathrm{CH}_{2} \mathrm{Cl}_{2} \ldots . . \mathrm{S} 15$

Figure S 16: Absorption versus concentration plots of 3. ............................ 15

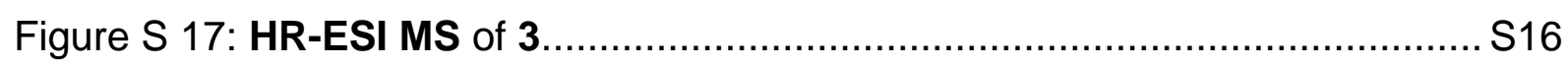

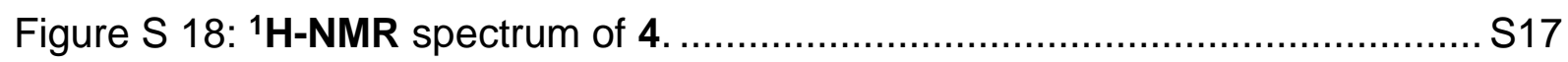

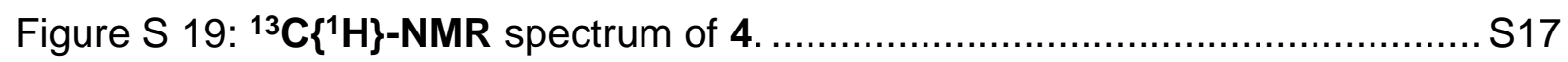

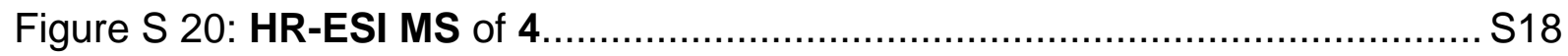

Figure S 21. ORTEP-representation of the solid state structure of 9. .............S19

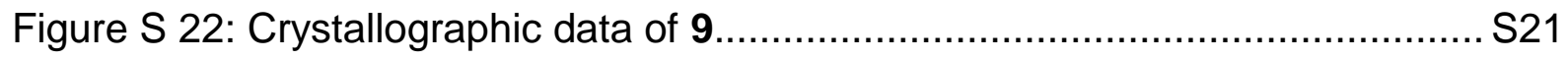

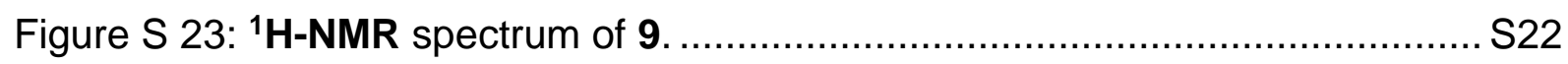

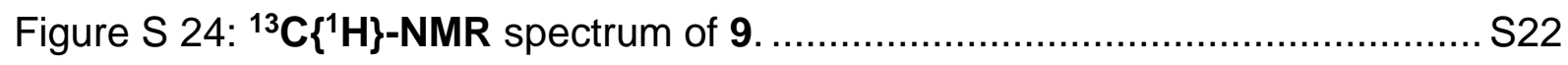

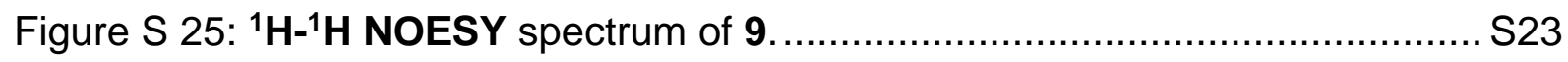

Figure S 26: Aromatic region of the ${ }^{1} \mathbf{H}-{ }^{1} \mathbf{H}$ NOESY spectrum of $9 \ldots \ldots \ldots \ldots \ldots \ldots . . . . . . . . . .323$

Figure S 27: Absorption spectra of a dilution series of 9 measured in $\mathrm{CH}_{2} \mathrm{Cl}_{2} \ldots . . \mathrm{S} 24$

Figure S 28: Absorption versus concentration plots of 9. ........................... $S 24$

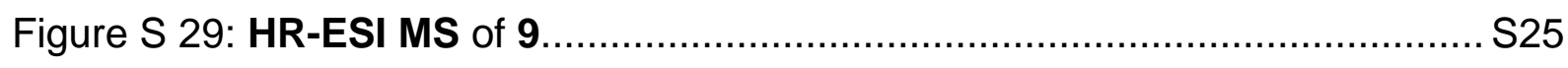

Figure S 30: ORTEP-representation of the solid state structure of 8 . ..............S26

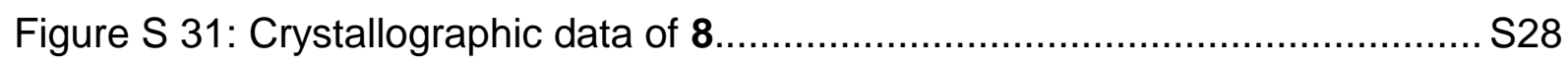




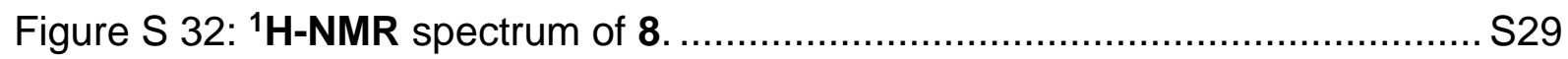

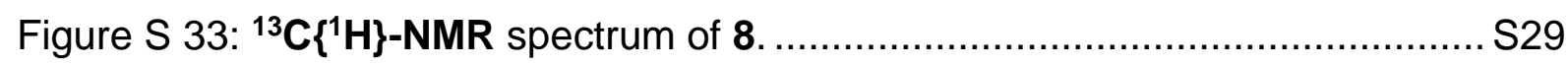

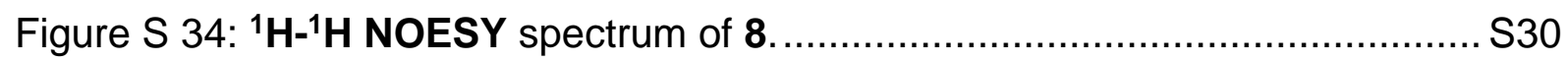

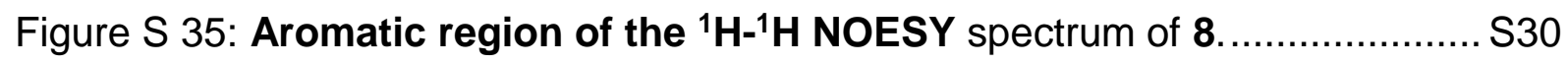

Figure S 36: Absorption spectra of a dilution series of 8 measured in $\mathrm{CH}_{2} \mathrm{Cl}_{2} \ldots . . \mathrm{S} 31$

Figure S 37: Absorption versus concentration plots of 8.............................. 31

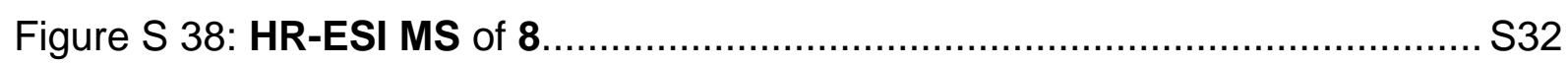

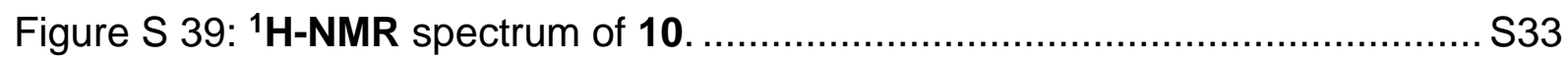

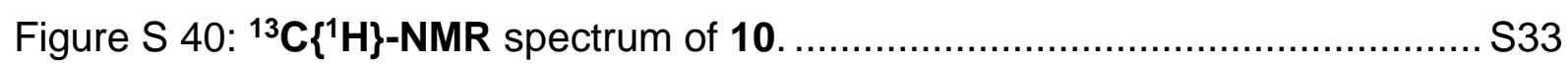

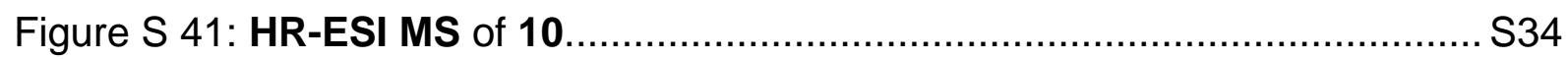

Figure $\mathrm{S} 42$ : Absorption spectrum of 10 measured in $\mathrm{CH}_{2} \mathrm{Cl}_{2} \ldots \ldots \ldots \ldots \ldots \ldots \ldots \ldots . . . . . . . . . . . . .535$

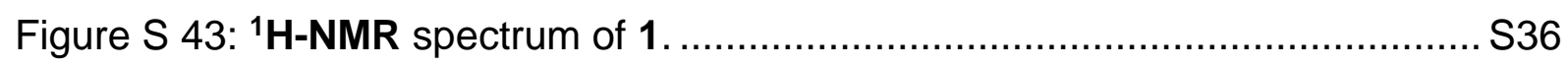

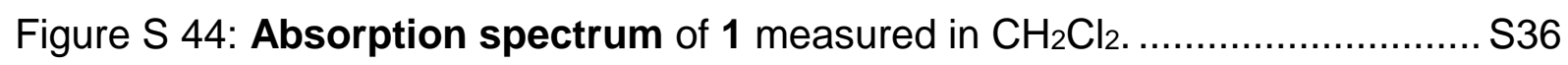

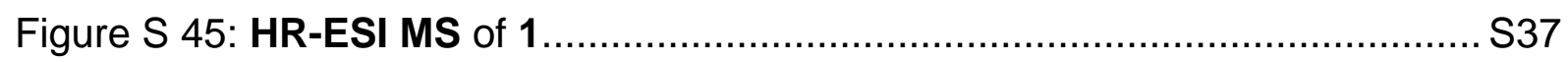

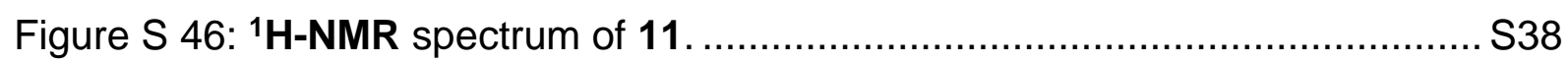

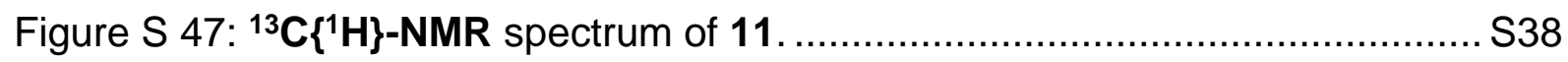

Figure $\mathrm{S} 48$ : Absorption spectrum of 11 measured in $\mathrm{CH}_{2} \mathrm{Cl}_{2} \ldots \ldots \ldots \ldots \ldots \ldots \ldots \ldots . . . . . . . . . . . .39$

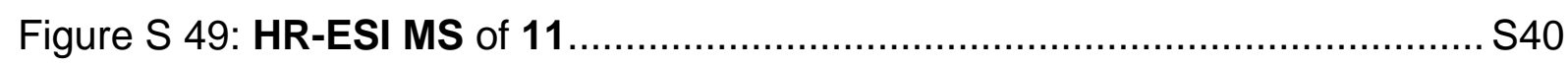

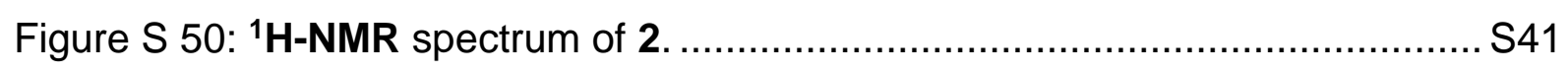

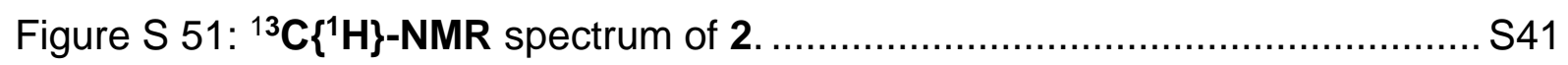

Figure S 52: Absorption spectrum of 2 measured in $\mathrm{CH}_{2} \mathrm{Cl}_{2} \ldots \ldots \ldots \ldots \ldots \ldots \ldots \ldots . . . . . . . . \ldots 42$

Figure S 53: HR-ESI MS of 2

Figure S 54: MCBJ Schematics .................................................................... 44 

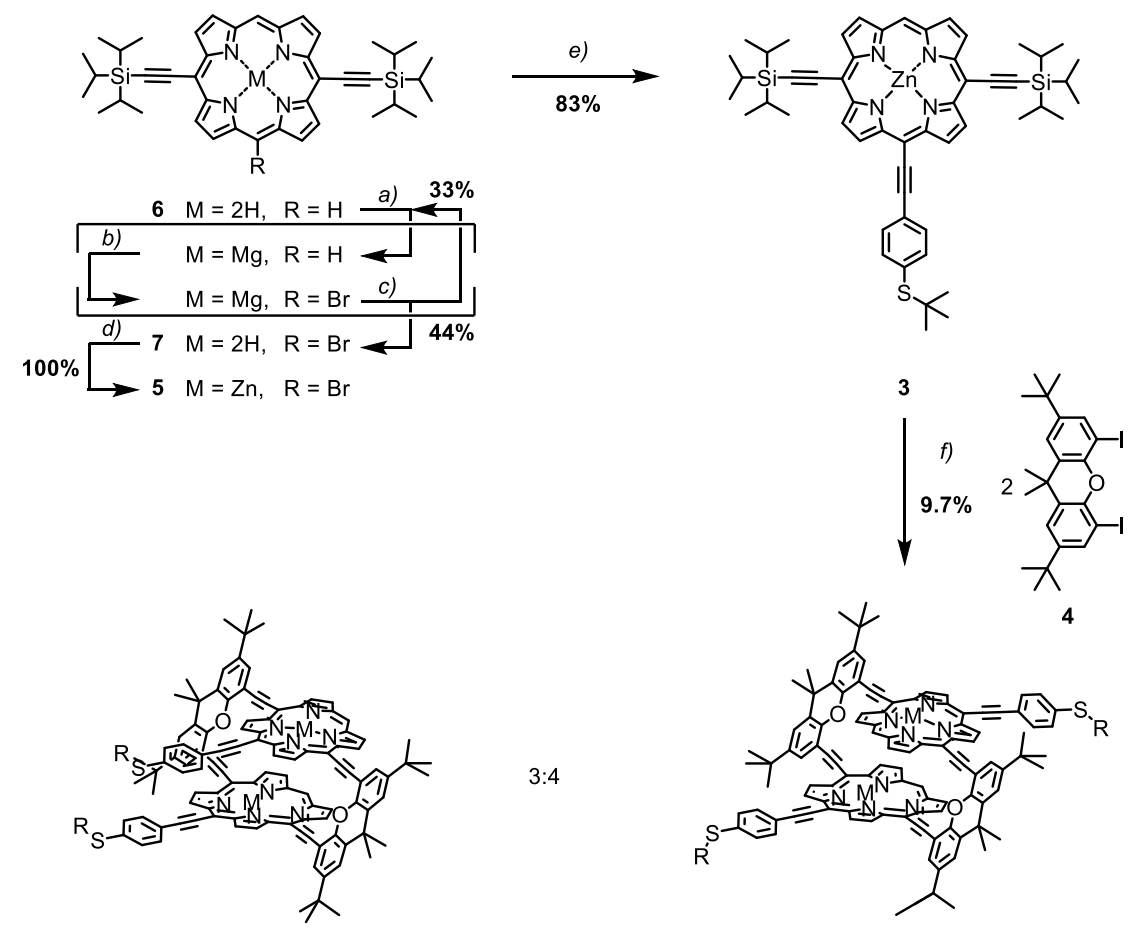

$\begin{array}{rlll}9 \mathrm{M}=\mathrm{Zn}, & \mathrm{R}=-\mathrm{C}\left(\mathrm{CH}_{3}\right)_{3} & \text { g) } \\ \text { h) } & \\ \mathbf{1 1} & \mathrm{M}=2 \mathrm{H} \quad \mathrm{R}=-\mathrm{COCH}_{3}\end{array} 55 \%$

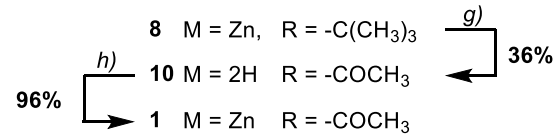

Synthetic overview. a) $\mathrm{Mgl}_{2}, \mathrm{CH}_{2} \mathrm{Cl}_{2}$, $\mathrm{NPr}_{2} \mathrm{Et}$, rt, $1 \mathrm{~h}$; b) $\left.\mathrm{NBS}, \mathrm{CHCl}_{3}, \mathrm{C}_{5} \mathrm{H}_{5} \mathrm{~N}, \mathrm{rt}, 16 \mathrm{~h} ; c\right) \mathrm{CF}_{3} \mathrm{CO}_{2} \mathrm{H}, \mathrm{CH}_{2} \mathrm{Cl}_{2}, \mathrm{rt}, 2 \mathrm{~h}$; d) $\mathrm{Zn}(\mathrm{OAc})_{2}, \mathrm{CH}_{2} \mathrm{Cl}_{2}, \mathrm{CH}_{3} \mathrm{OH}$, rt, $2 \mathrm{~h}$; e) tert-butyl-(4-ethynylphenyl)sulfane, $\mathrm{Pd}\left(\mathrm{PPh}_{3}\right)_{4}$, $\mathrm{Cul}$, THF, $\mathrm{NEt} 3,50{ }^{\circ} \mathrm{C}, 16 \mathrm{~h}$; f) 1) TBAF, THF, rt, $1 \mathrm{~h}$; 2) $\mathrm{Pd}\left(\mathrm{PPh}_{3}\right)_{4}$, Cul, THF, NEt, $80^{\circ} \mathrm{C}$ (sealed tube), $\left.16 \mathrm{~h} ; \mathrm{g}\right) \mathrm{Bi}\left(\mathrm{OSO}_{2} \mathrm{CF}_{3}\right)_{3}, \mathrm{AcCl}, \mathrm{rt}, 12 \mathrm{~h}$; h) $\mathrm{Zn}(\mathrm{OAc})_{2}, \mathrm{CH}_{2} \mathrm{Cl}_{2}, \mathrm{CH}_{3} \mathrm{OH}, \mathrm{rt}, 1 \mathrm{~d}$. 


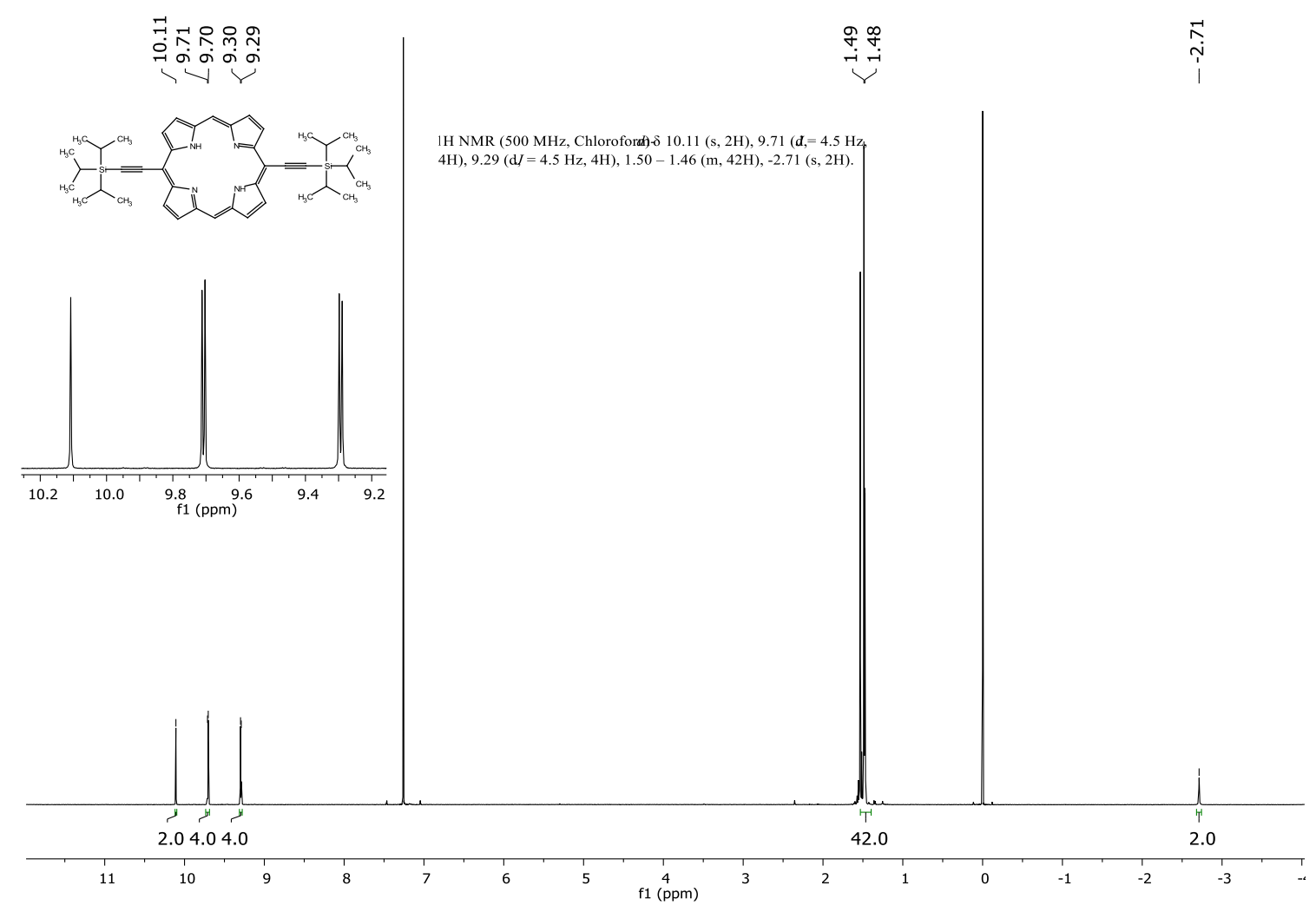

Figure $S$ 1: ${ }^{1} \mathbf{H}$-NMR spectrum of 6 .

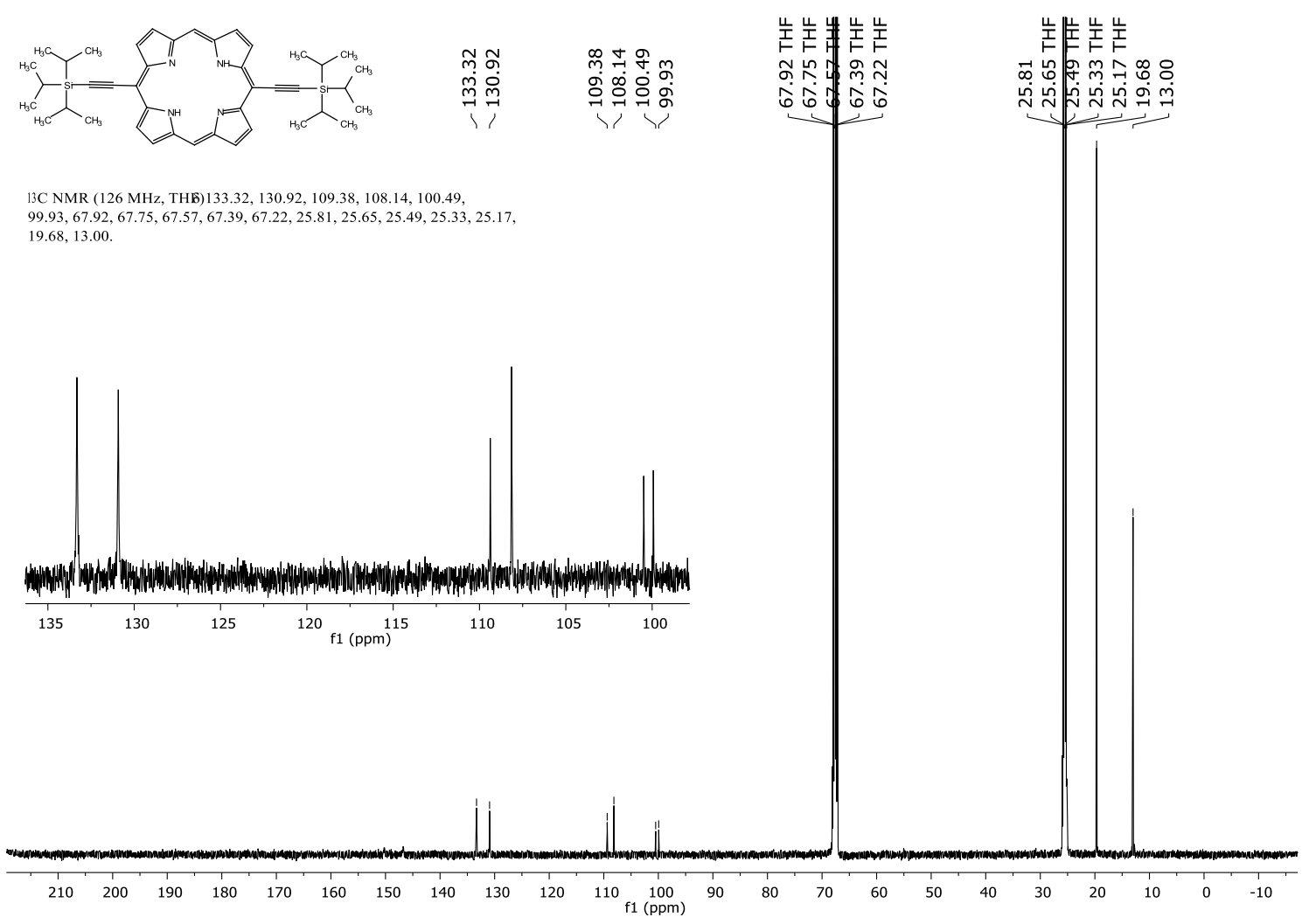

Figure $S 2:{ }^{13} \mathrm{C}\left\{{ }^{1} \mathrm{H}\right\}-\mathrm{NMR}$ spectrum of 6. 


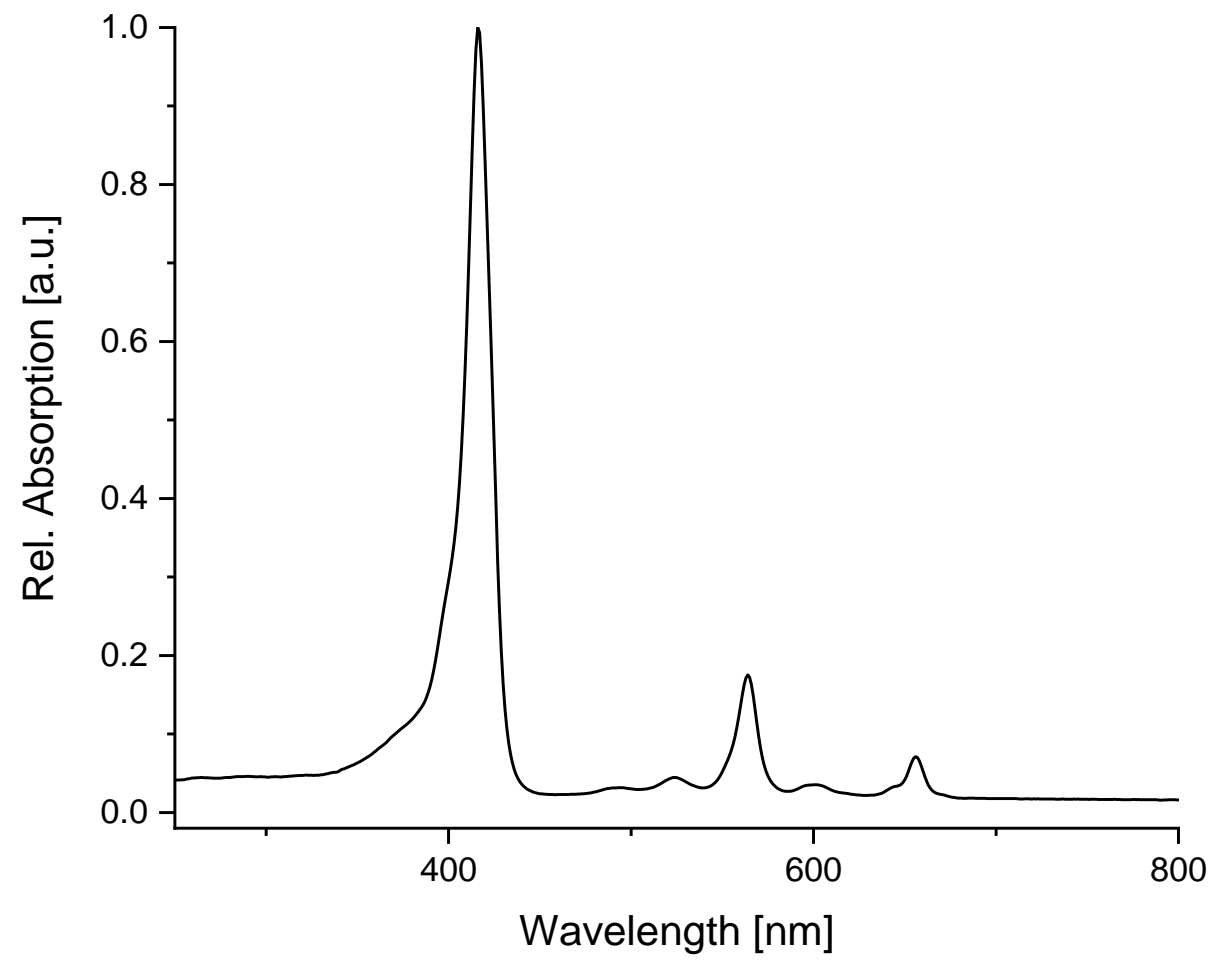

Figure $S$ 3: Absorption spectrum of 6 measured in $\mathrm{CH}_{2} \mathrm{Cl}_{2}$. 


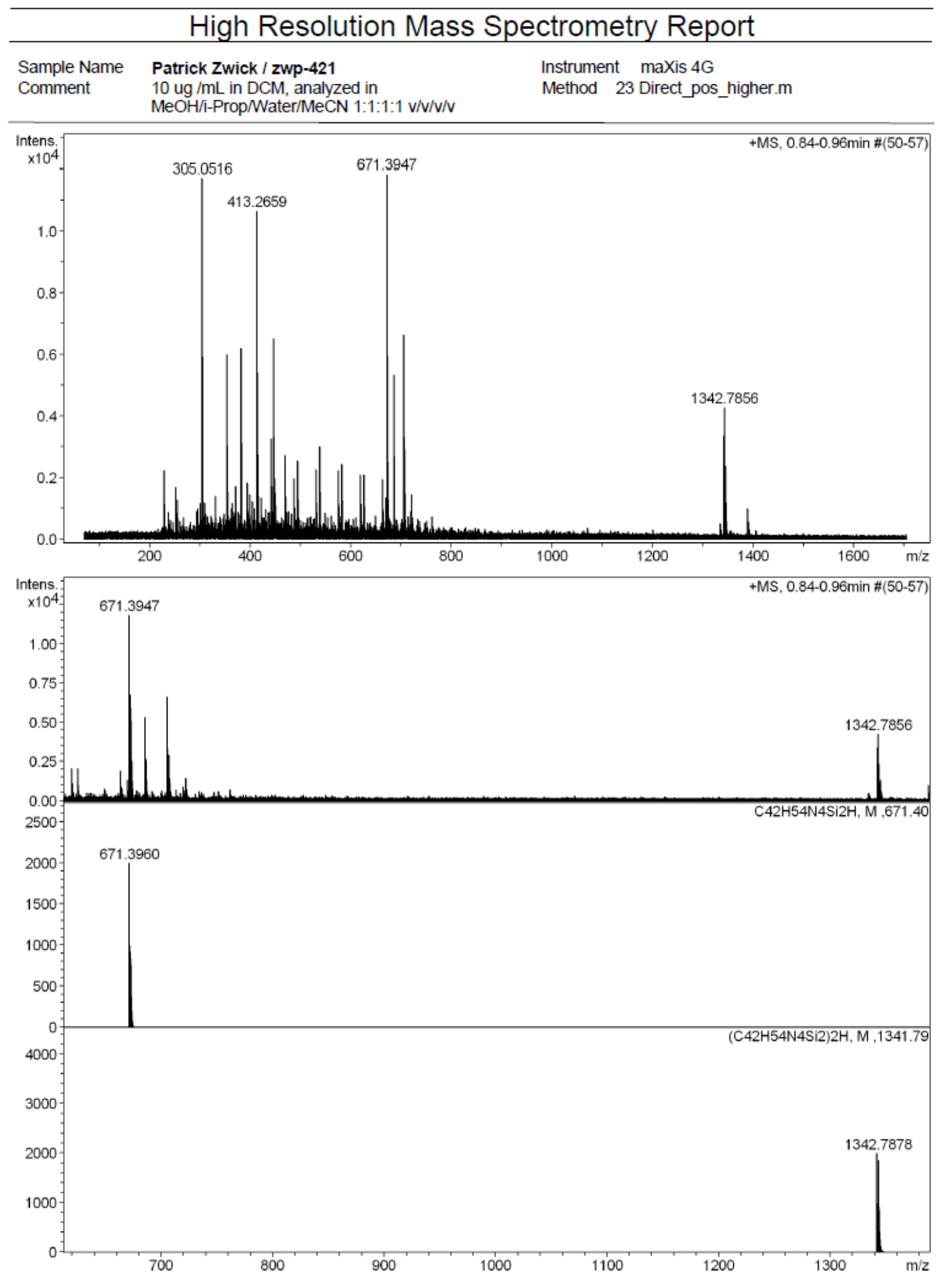

Figure S 4: HR-ESI MS of 6. 


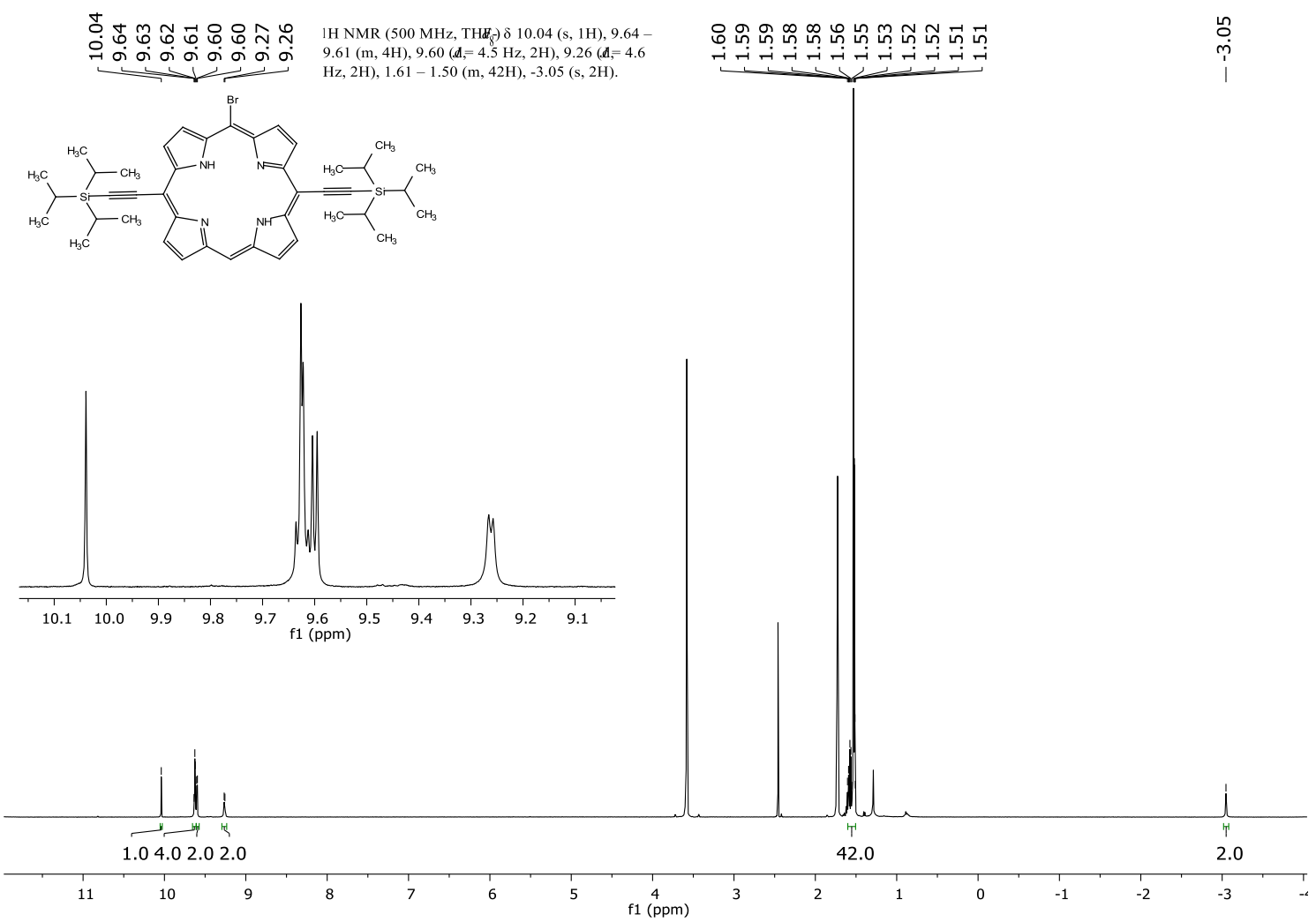

Figure S 5: ${ }^{1} \mathrm{H}-\mathrm{NMR}$ spectrum of 7.
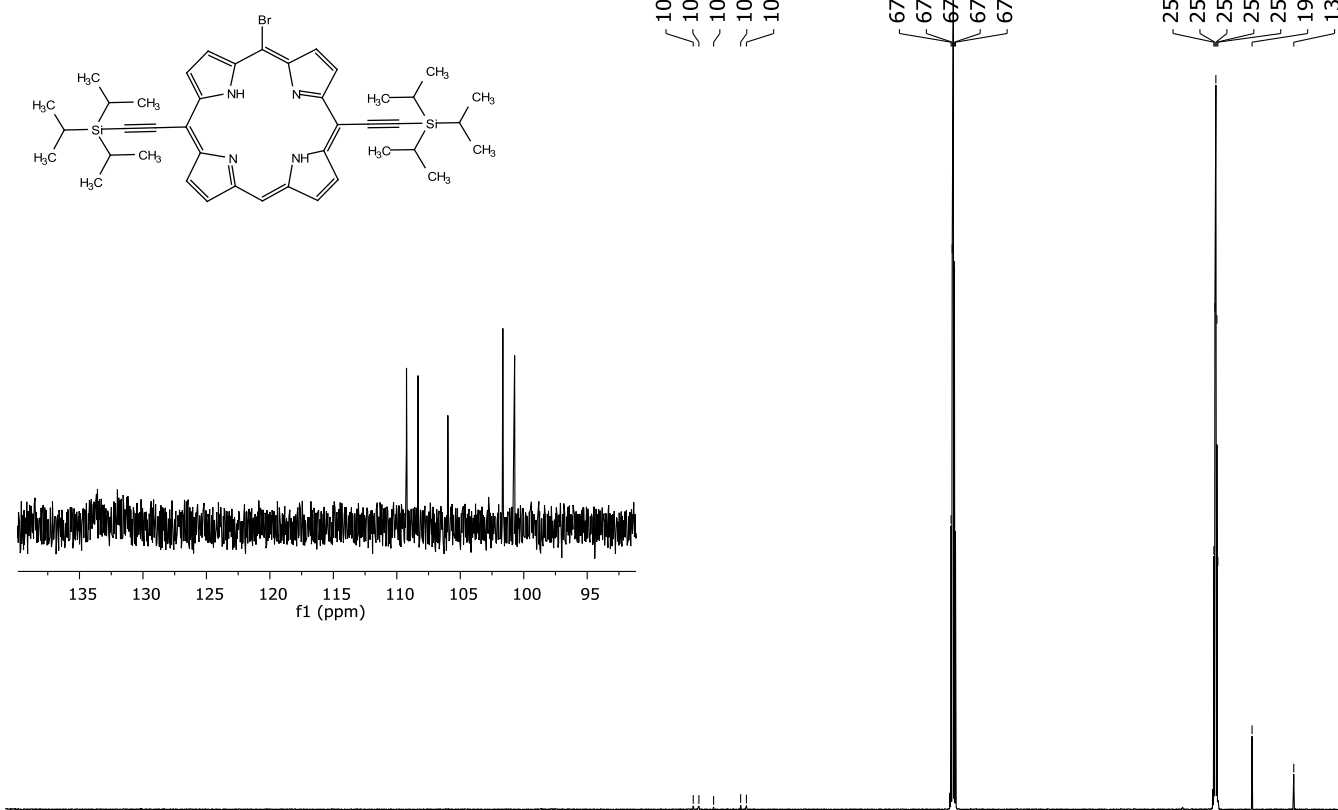

$\begin{array}{lllllllllll}210 & 200 & 190 & 180 & 170 & 160 & 150 & 140 & 130 & 120 & 110 \begin{array}{c}100 \\ \mathrm{f} 1(\mathrm{ppm})\end{array}\end{array}$

Figure $S 6:{ }^{13} \mathrm{C}\left\{{ }^{1} \mathrm{H}\right\}-\mathrm{NMR}$ spectrum of 7 . 


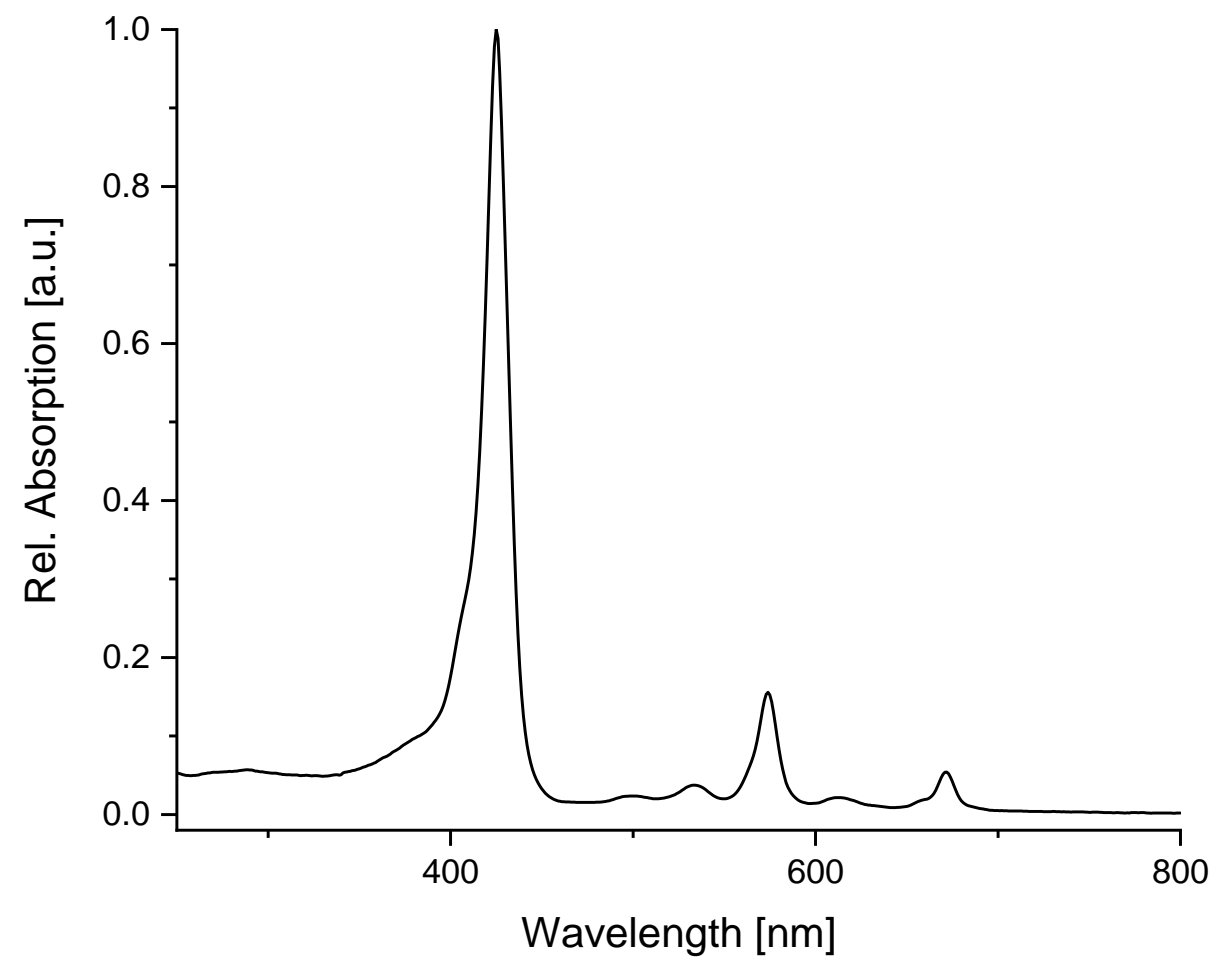

Figure $S$ 7: Absorption spectrum of 7 measured in $\mathrm{CH}_{2} \mathrm{Cl}_{2}$. 

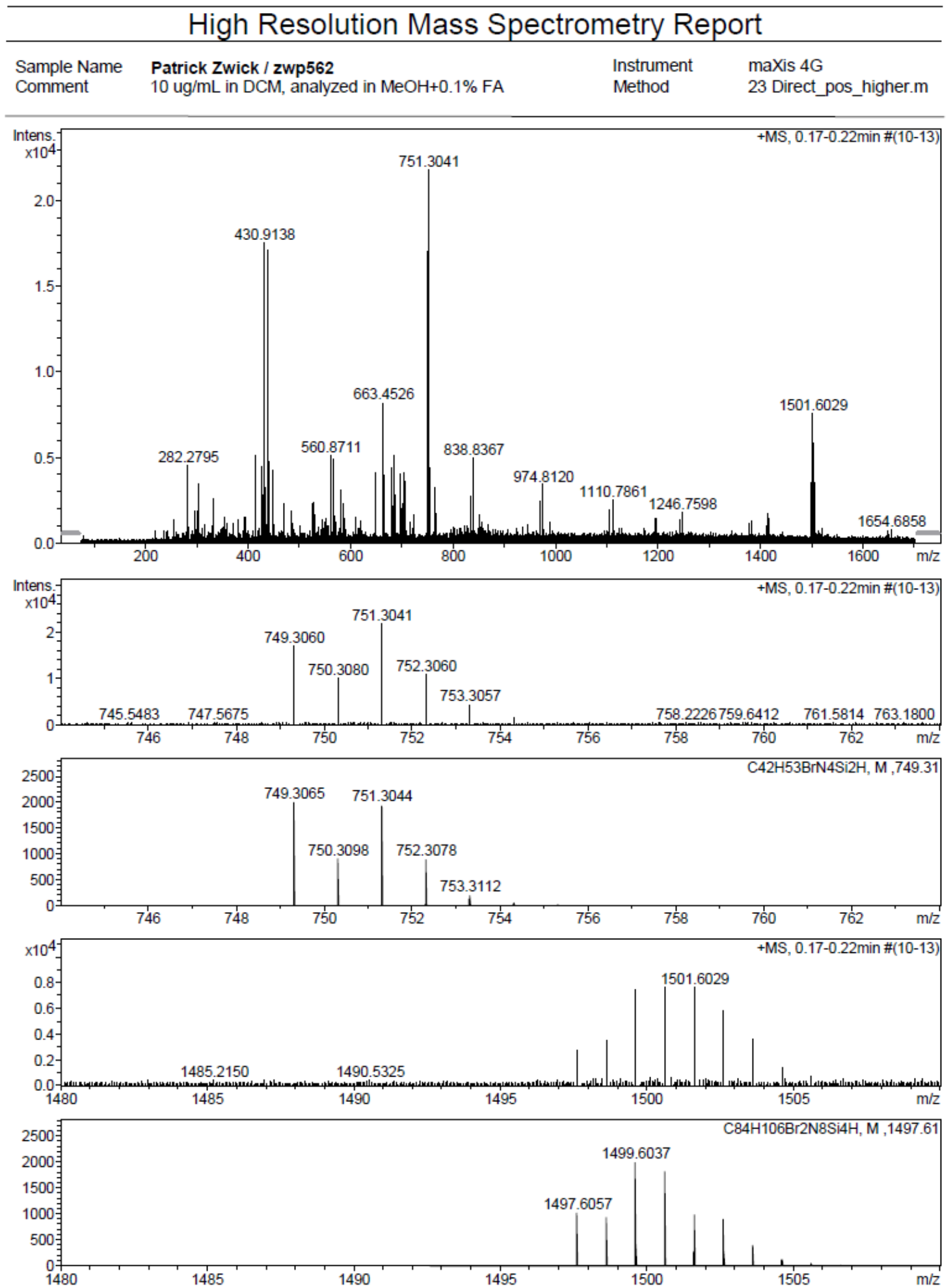

Figure S 8: HR-ESI MS of 7. 

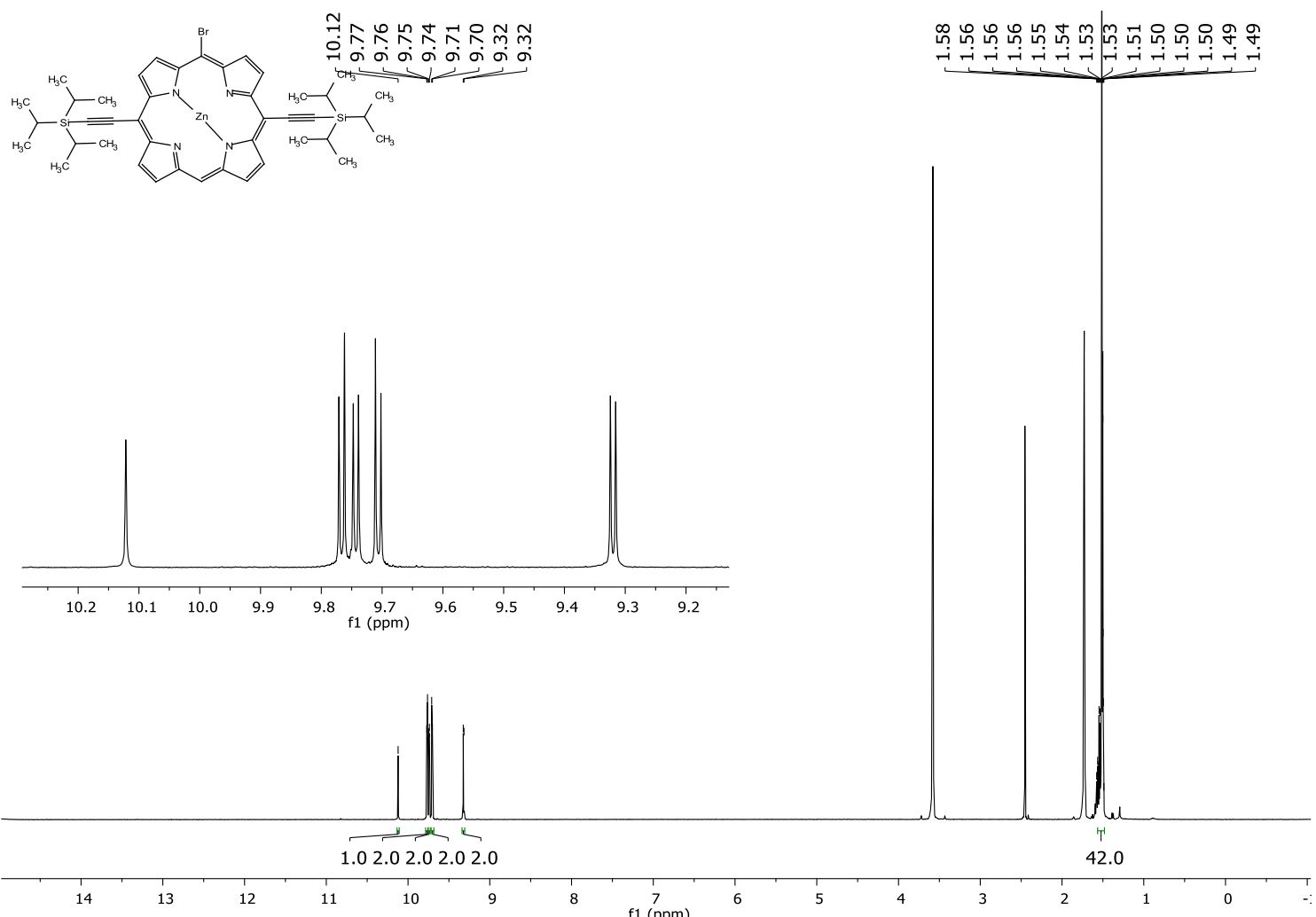

Figure S 9: ${ }^{1} \mathbf{H}-\mathrm{NMR}$ spectrum of 5.

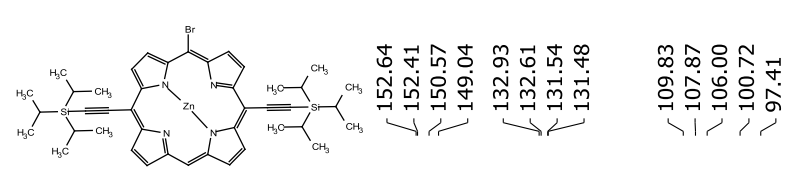

13C NMR (126 MHz, THE) $152.64,152.41,150.57,149.04,132.93,132.61,131.54,131.48,109.83$

$107.87,106.00,100.72,97.41,66.76,66.59,66.41,66.24,66.06,24.65,24.49,24.33,24.17,24.01,18.55$,
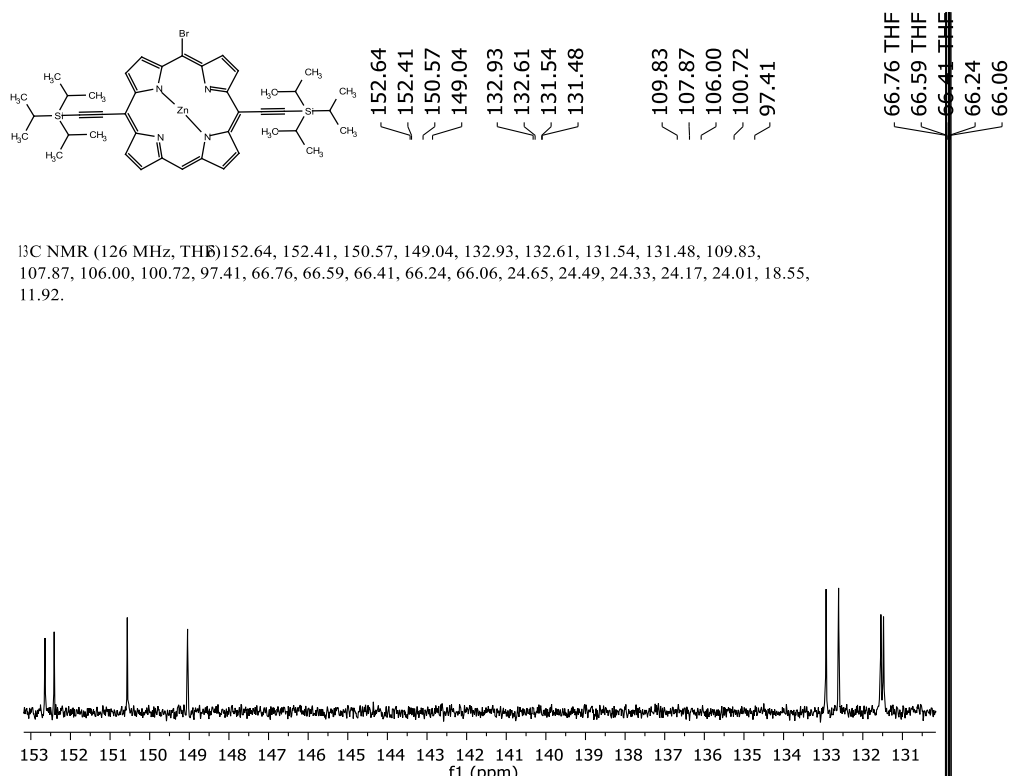

岸崖崖

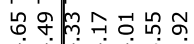

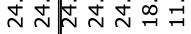

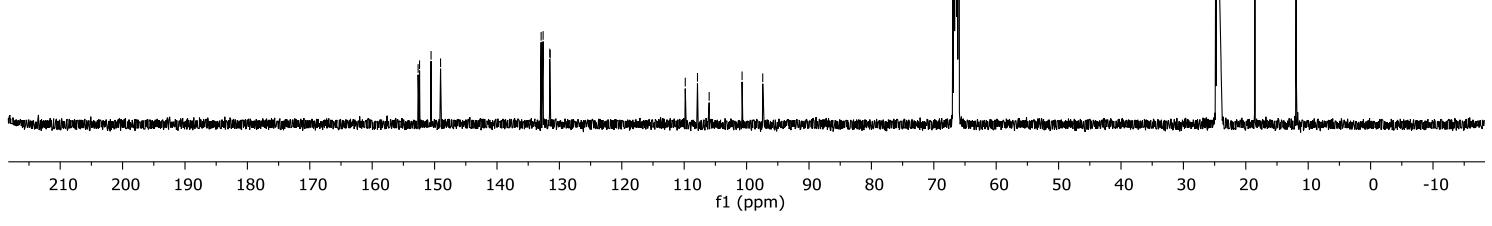

Figure $S$ 10. ${ }^{13} \mathrm{C}\left\{{ }^{1} \mathrm{H}\right\}-\mathrm{NMR}$ spectrum of 5 . 


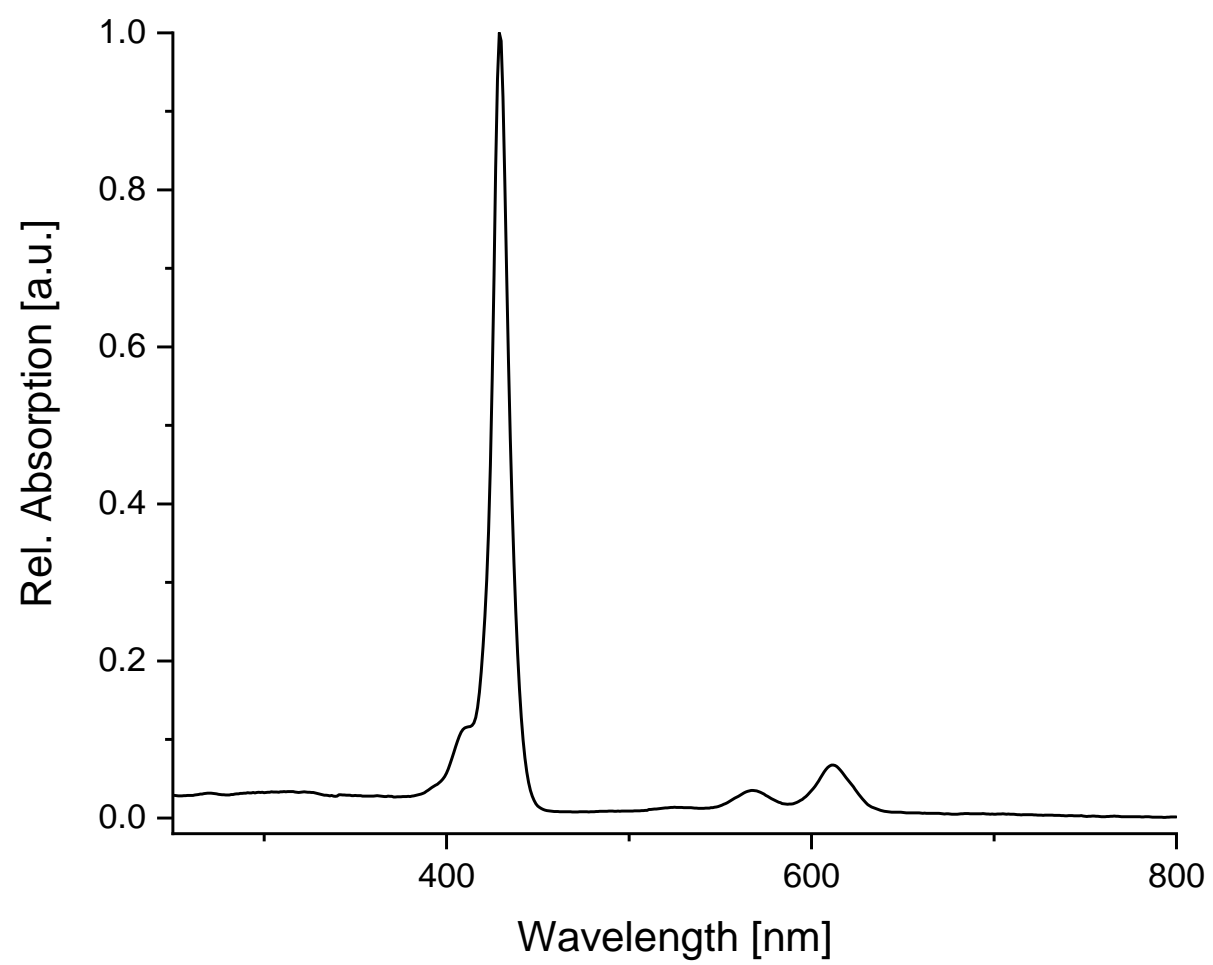

Figure $S$ 11: Absorption spectrum of 5 measured in $\mathrm{CH}_{2} \mathrm{Cl}_{2}$. 


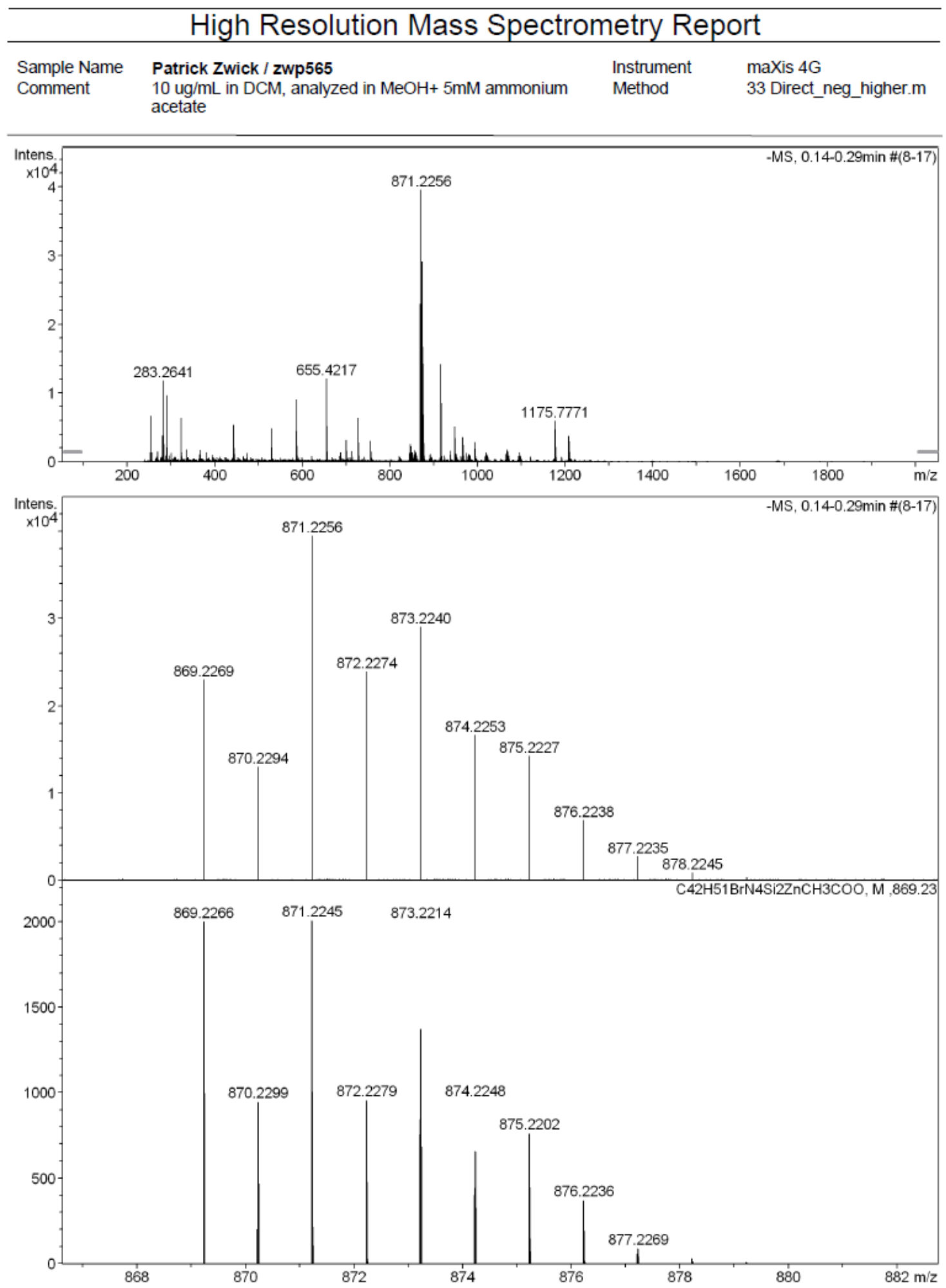

Figure S 12: HR-ESI MS of 5. 


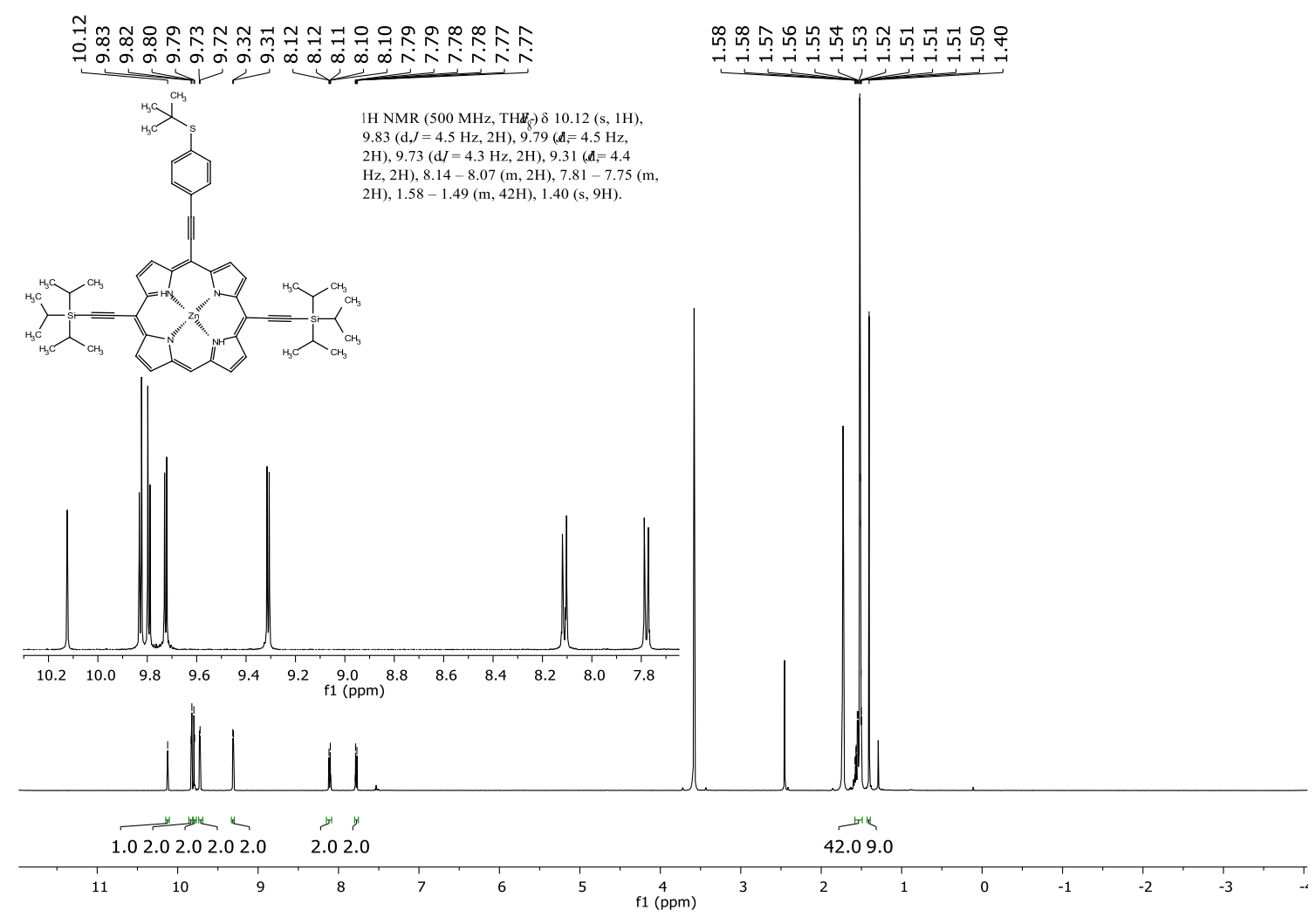

Figure $S$ 13: ${ }^{1} \mathrm{H}-\mathrm{NMR}$ spectrum of 3.
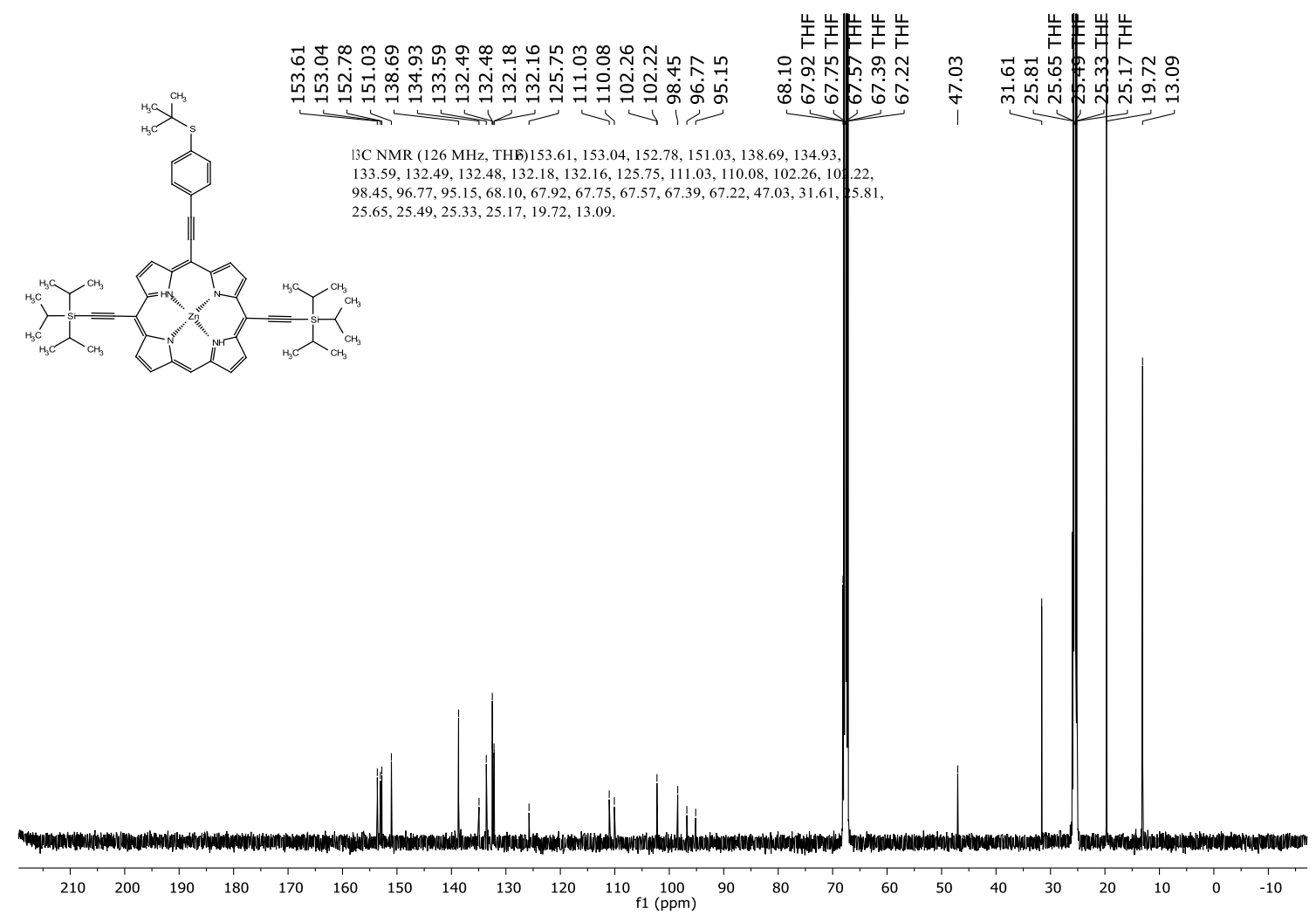

Figure $S$ 14: ${ }^{13} \mathbf{C}\left\{{ }^{1} \mathrm{H}\right\}-N M R$ spectrum of 3 . 


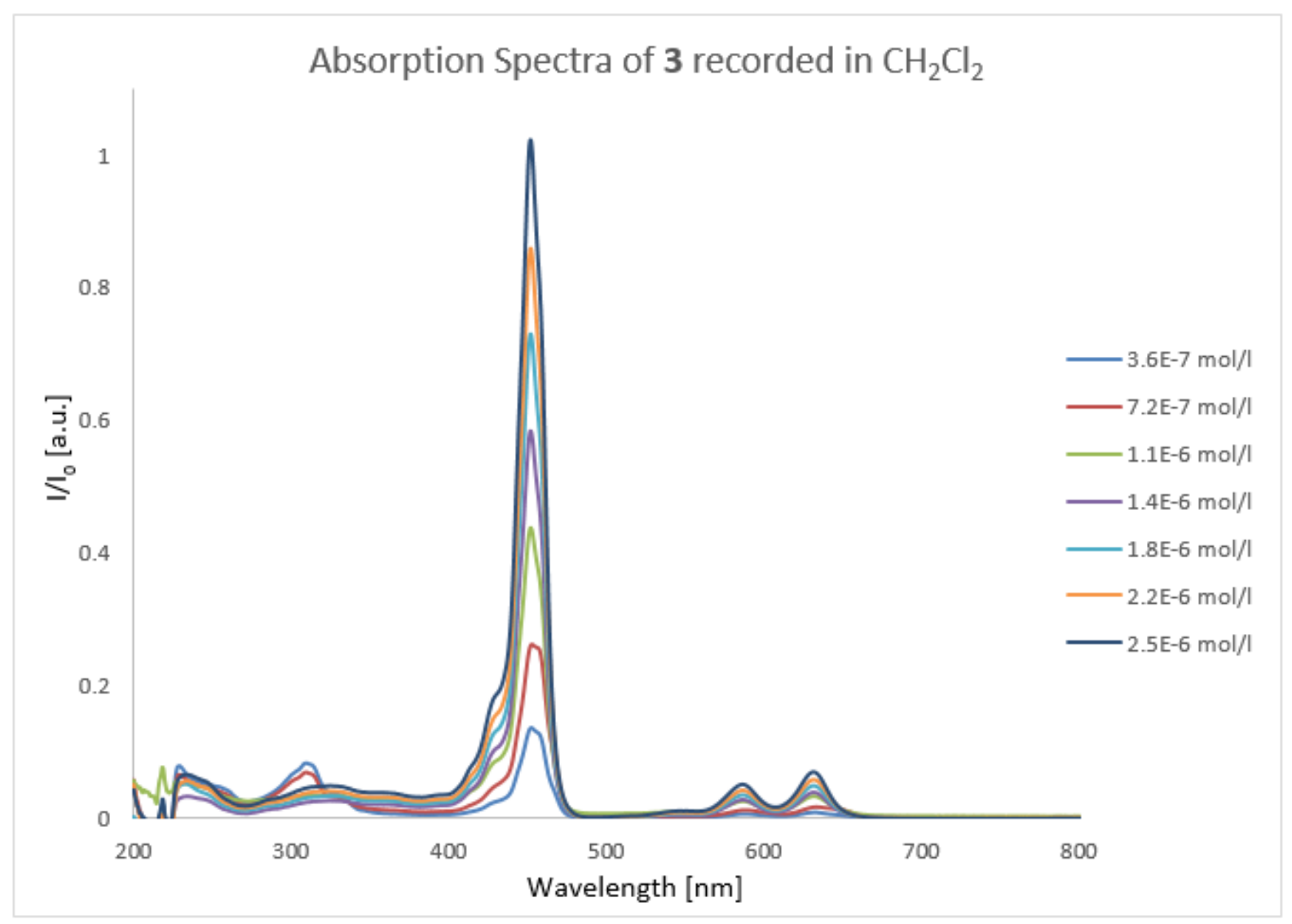

Figure S 15: Absorption spectra of a dilution series of 3 measured in $\mathrm{CH}_{2} \mathrm{Cl}_{2}$.
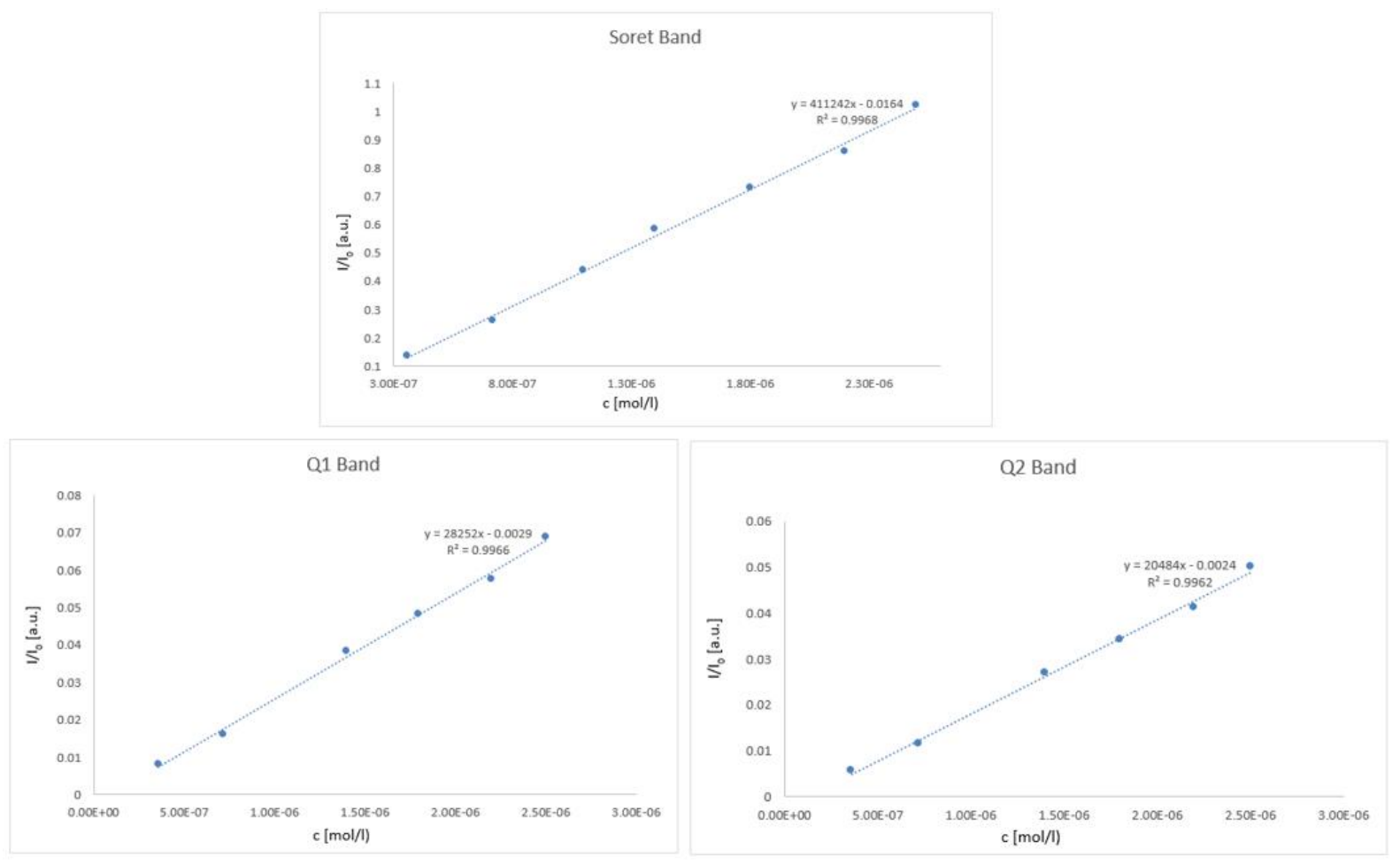

Figure S 16: Absorption versus concentration plots of 3 for the Soret, Q1, and Q2 bands. 


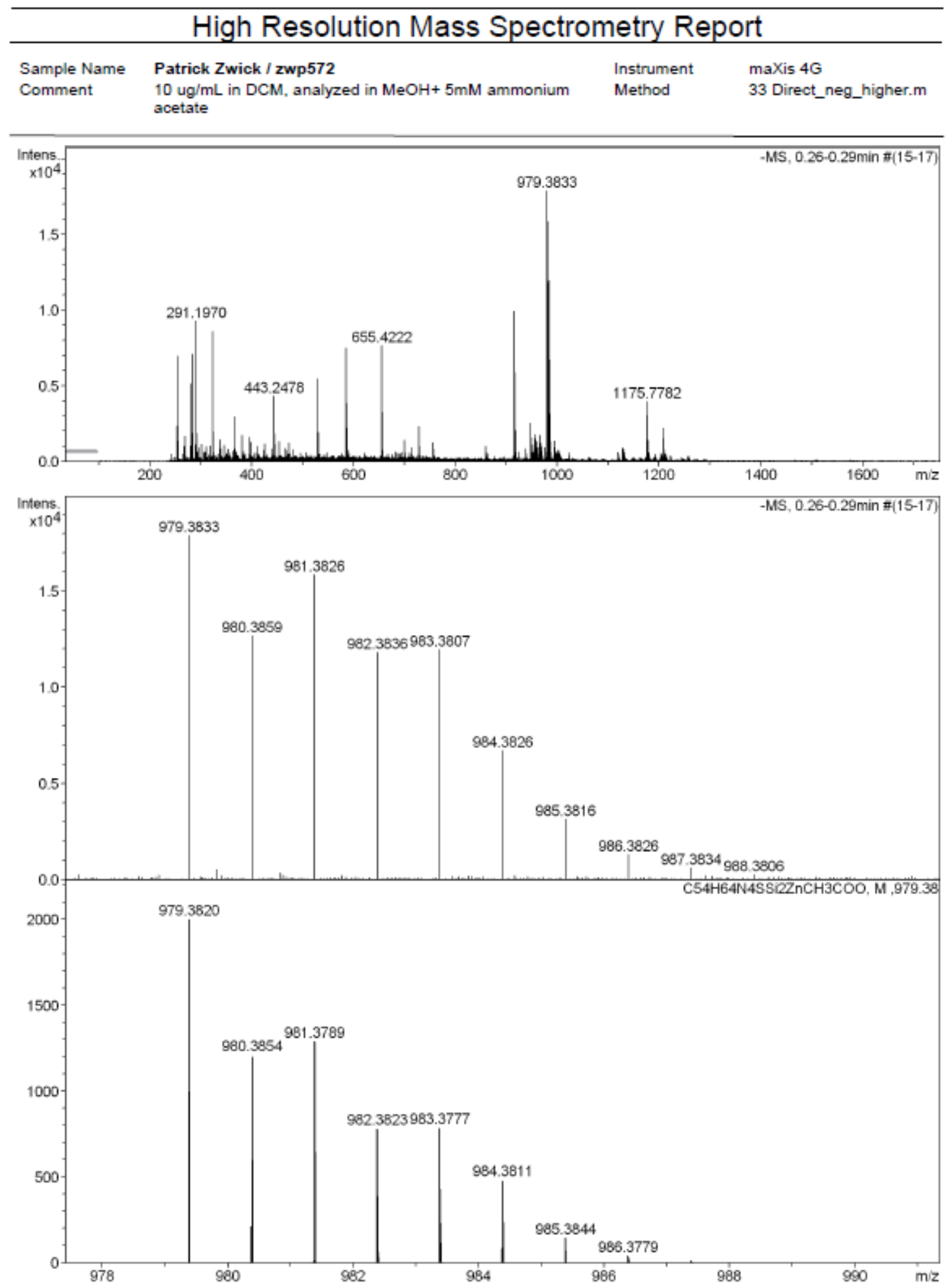

Figure S 17: HR-ESI MS of 3. 


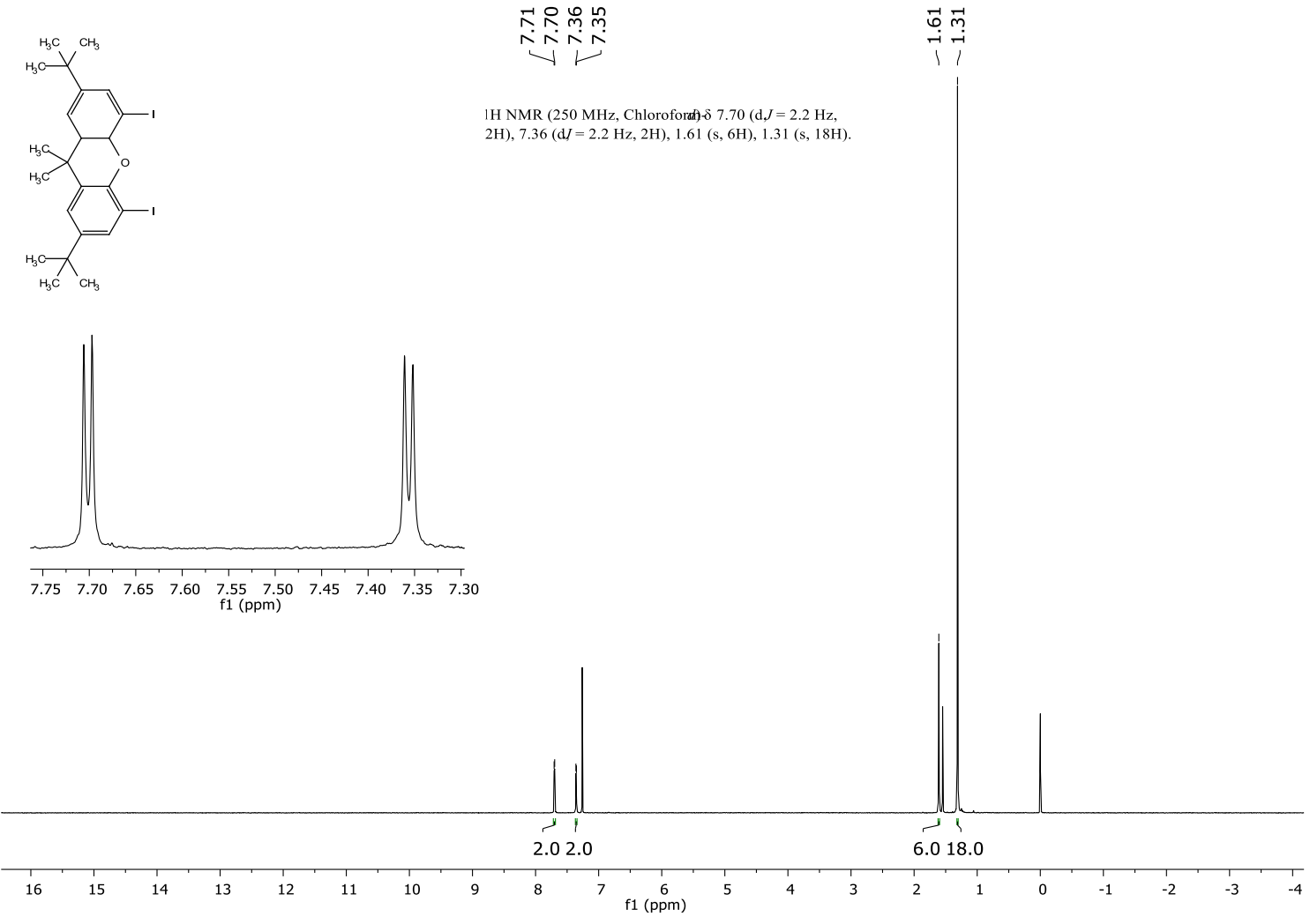

Figure $S$ 18: ${ }^{1} \mathrm{H}-\mathrm{NMR}$ spectrum of 4.
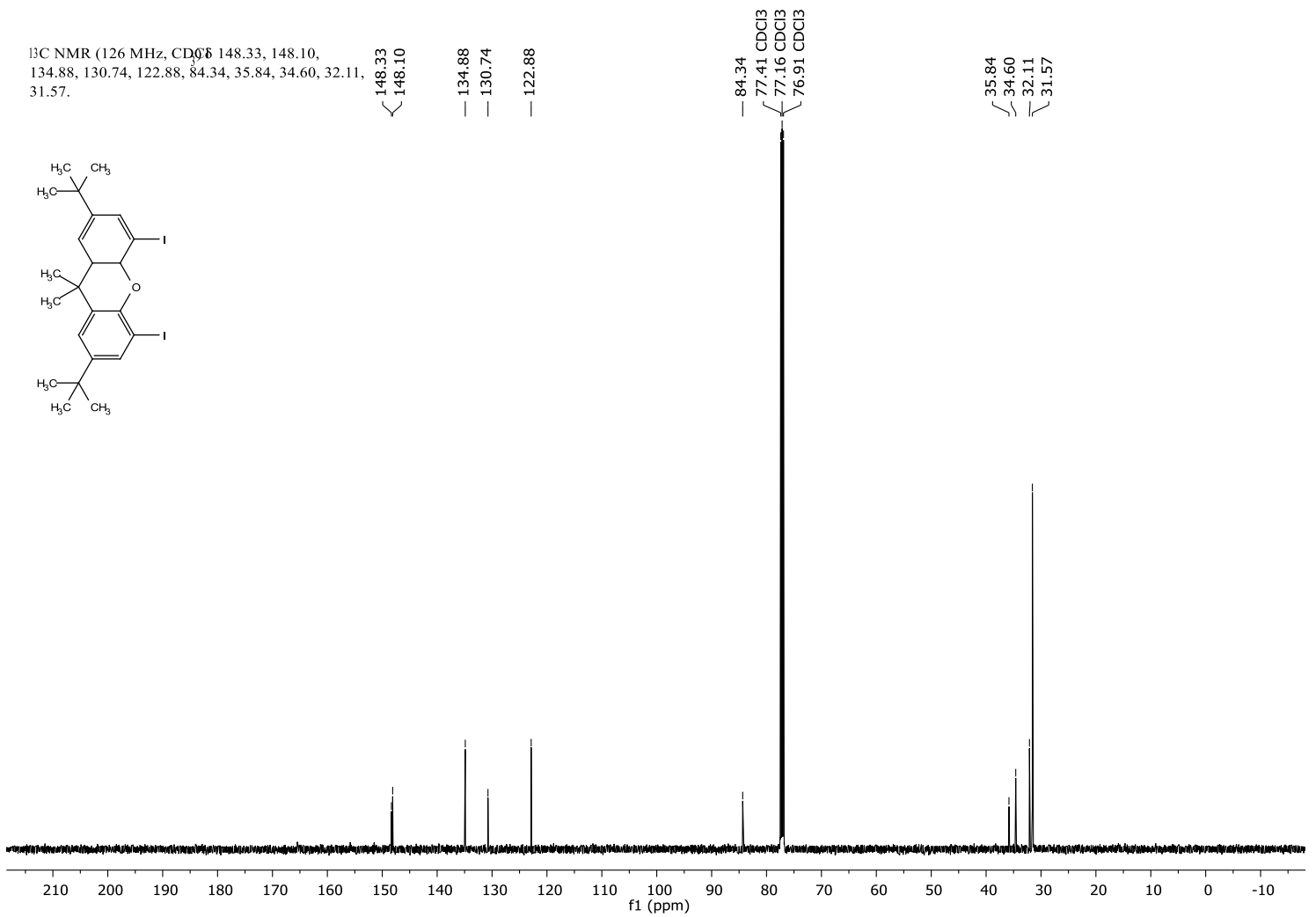

Figure $S$ 19: ${ }^{13} \mathbf{C}\left\{{ }^{1} \mathrm{H}\right\}-N M R$ spectrum of 4 . 


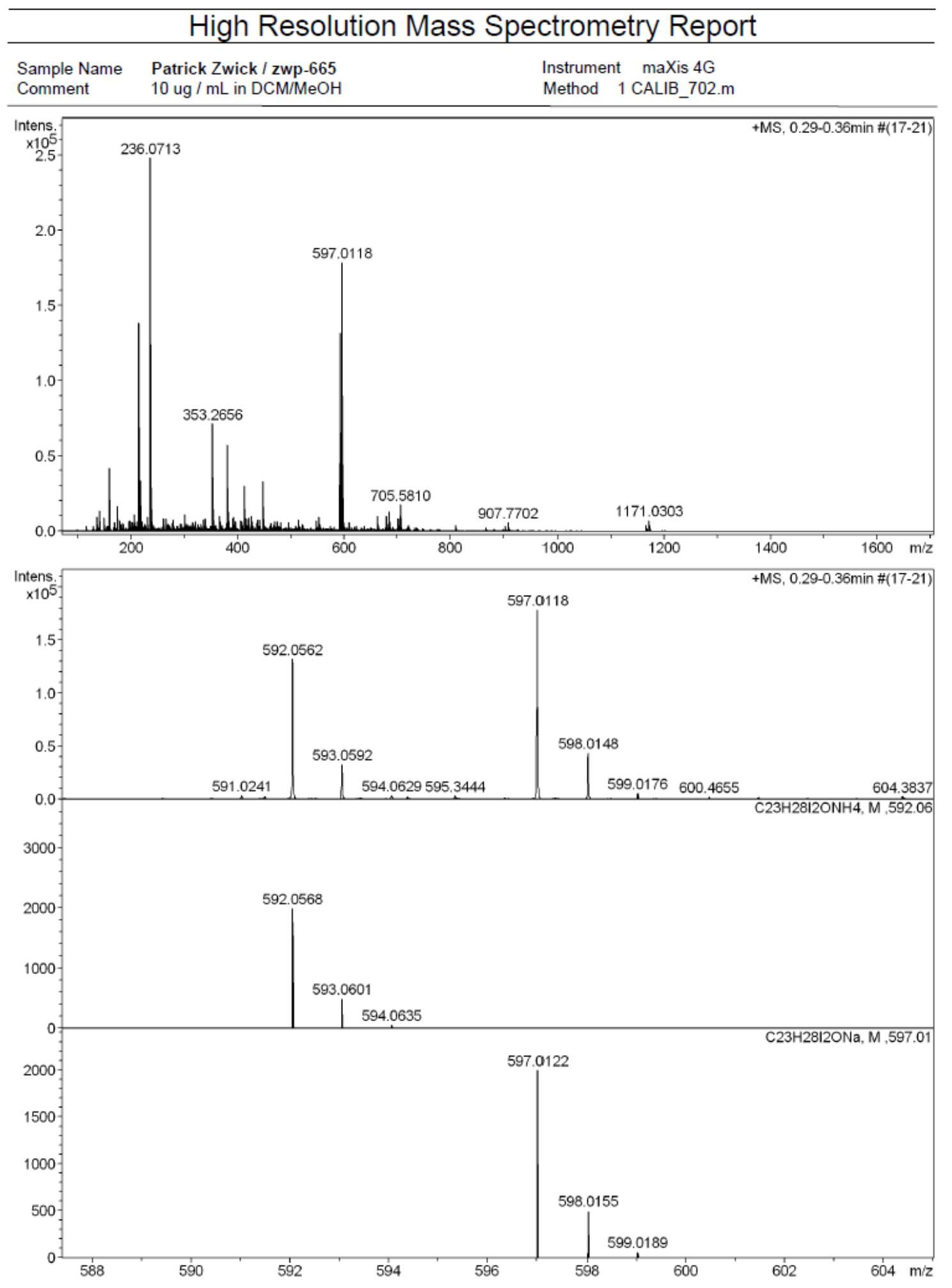

Figure S 20: HR-ESI MS of 4. 


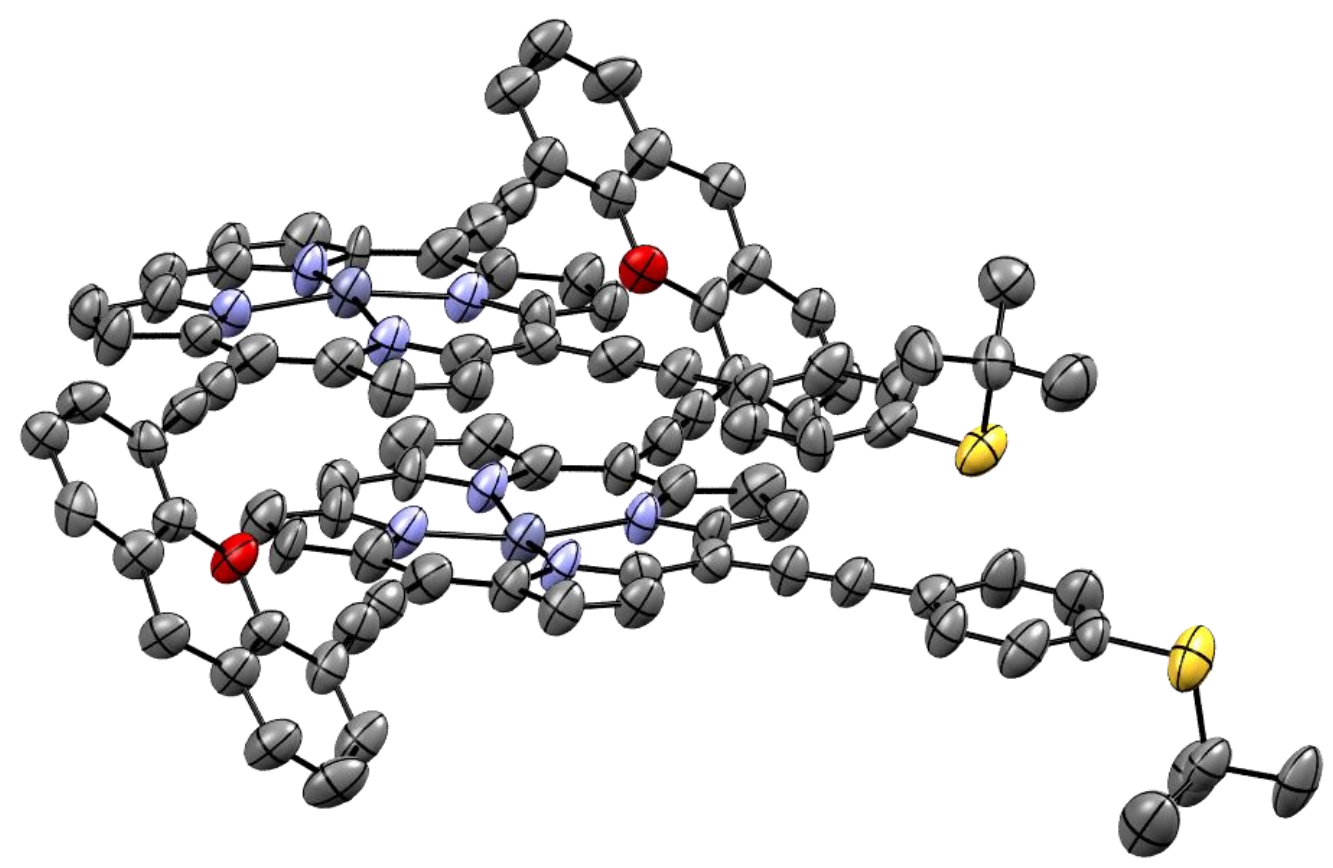

Figure S 21. ORTEP-representation of the solid state structure of 9 . Thermal ellipsoids are plotted on a $50 \%$ probability level. Solvent molecules and bulky groups are omitted for clarity. Single crystals suitable for $\mathrm{x}$-ray analysis were obtained by slow diffusion of methanol into a tetrahydrofuran (THF) solution of 9 using the toplayering method. 
Table S1 Crystal data and structure refinement for 9.

$\begin{array}{ll}\text { Empirical formula } & \mathrm{C}_{126} \mathrm{H}_{116} \mathrm{~N}_{8} \mathrm{O}_{4} \mathrm{~S}_{2} \mathrm{Zn}_{2} \\ \text { Formula weight } & 2001.12 \\ \text { Temperature/K } & 180.0 \\ \text { Crystal system } & \text { triclinic } \\ \text { Space group } & P 1 \\ \mathrm{a} / \AA & 11.6608(16) \\ \mathrm{b} / \AA & 17.886(3) \\ \mathrm{c} / \AA & 28.102(6) \\ \alpha /{ }^{\circ} & 91.302(15) \\ \beta /{ }^{\circ} & 91.892(15) \\ \gamma /{ }^{\circ} & 103.900(11) \\ \mathrm{Volume} / \AA^{3} & 5683.4(17) \\ \mathrm{Z} & 2 \\ \rho_{\text {calcg }} / \mathrm{cm}^{3} & 1.169 \\ \mu / \mathrm{mm}^{-1} & 0.813 \\ \mathrm{~F}(000) & 2104.0 \\ \text { Crystal size } / \mathrm{mm}^{3} & 0.16 \times 0.03 \times 0.02 \\ \text { Radiation } & \mathrm{GaK} \alpha(\lambda=1.34143)\end{array}$

$2 \Theta$ range for data collection/ ${ }^{\circ} 6.8$ to 85.224

$\begin{array}{ll}\text { Index ranges } & -11 \leq \mathrm{h} \leq 4,-15 \leq \mathrm{k} \leq 18,-28 \leq 1 \leq 28 \\ \text { Reflections collected } & 25912 \\ \text { Independent reflections } & 11703\left[\mathrm{R}_{\text {int }}=0.1236, \mathrm{R}_{\text {sigma }}=0.2516\right] \\ \text { Indep. refl. with } \mathrm{I} \geq 2 \sigma(\mathrm{I}) & 4712 \\ \text { Data/restraints/parameters } & 11703 / 22 / 1283 \\ \text { Goodness-of-fit on } \mathrm{F}^{2} & 0.912 \\ \text { Final } \mathrm{R} \text { indexes }[\mathrm{I} \geq 2 \sigma(\mathrm{I})] & \mathrm{R}_{1}=0.0773, \mathrm{wR}_{2}=0.1808 \\ \text { Final } \mathrm{R} \text { indexes [all data] } & \mathrm{R}_{1}=0.2051, \mathrm{wR}_{2}=0.2211 \\ \text { Largest diff. peak/hole / e } \AA^{-3} 0.51 /-0.41 \\ \text { CCDC number } & 2019592\end{array}$

Although the crystal structure of 9could be solved, the crystallographic data of this compound is not fully satisfying due to poor crystal quality. Even with the highly intensive X-ray radiation of the Ga MetalJet X-ray source only weak peak intensities were observed. Especially for high diffraction angles $\left(2 \Theta>85^{\circ}\right)$ there weren't any reasonable signals at all. One tert-butyl group and one coordinating THF molecule were refined as disordered over two positions. The solvent accessible void in the packing was handled by using the OLEX-implemented solvent mask algorithm(aka SQEEZE). The void has a volume of approx. $470 \AA^{3}$ containing 168 electrons. Latter can be approximated as four additional molecules of non-coordinating THF. 


\section{checkCIF/PLATON report}

Structure factors have been supplied for datablock(s) zwp-658

THIS REPORT IS FOR GUIDANCE ONLY. IF USED AS PART OF A REVIEW PROCEDURE FOR PUBLICATION, IT SHOULD NOT REPLACE THE EXPERTISE OF AN EXPERIENCED CRYSTALLOGRAPHIC REFEREE.

No syntax errors found. CIF dictionary Interpreting this report

\section{Datablock: zwp-658}

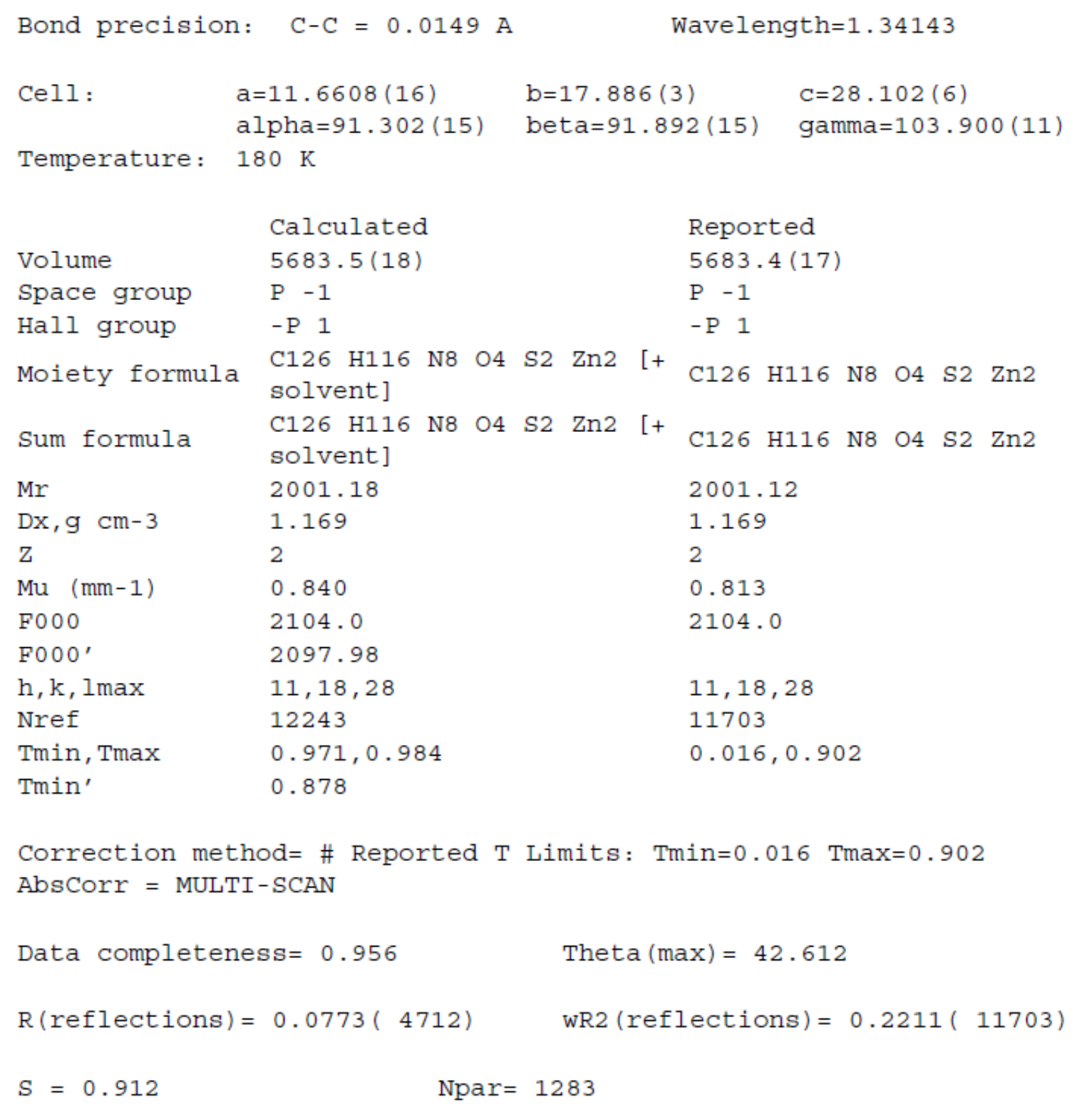

Figure S 22: Crystallographic data of 9. 


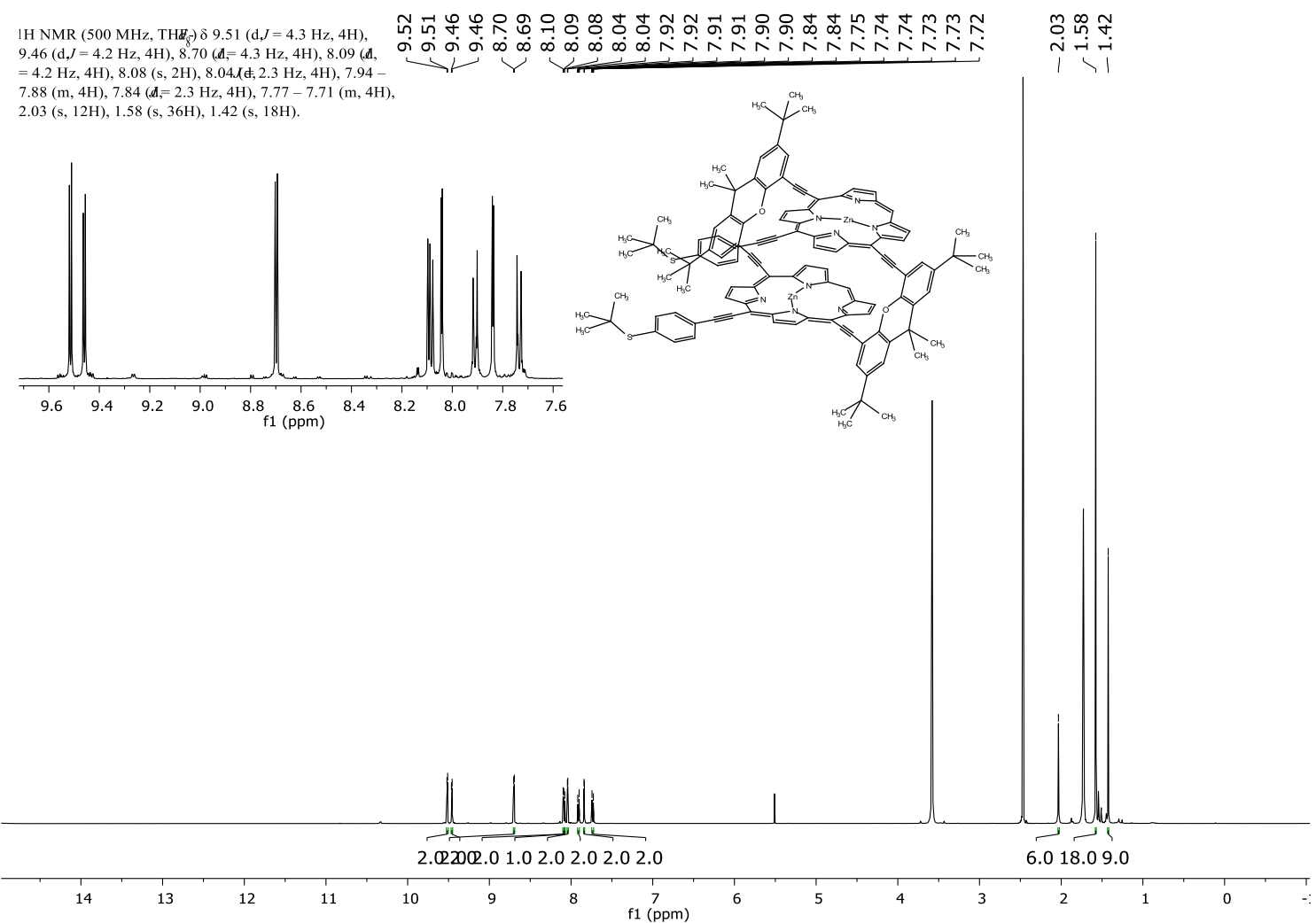

Figure $S$ 23: ${ }^{1} \mathbf{H}-\mathrm{NMR}$ spectrum of 9.

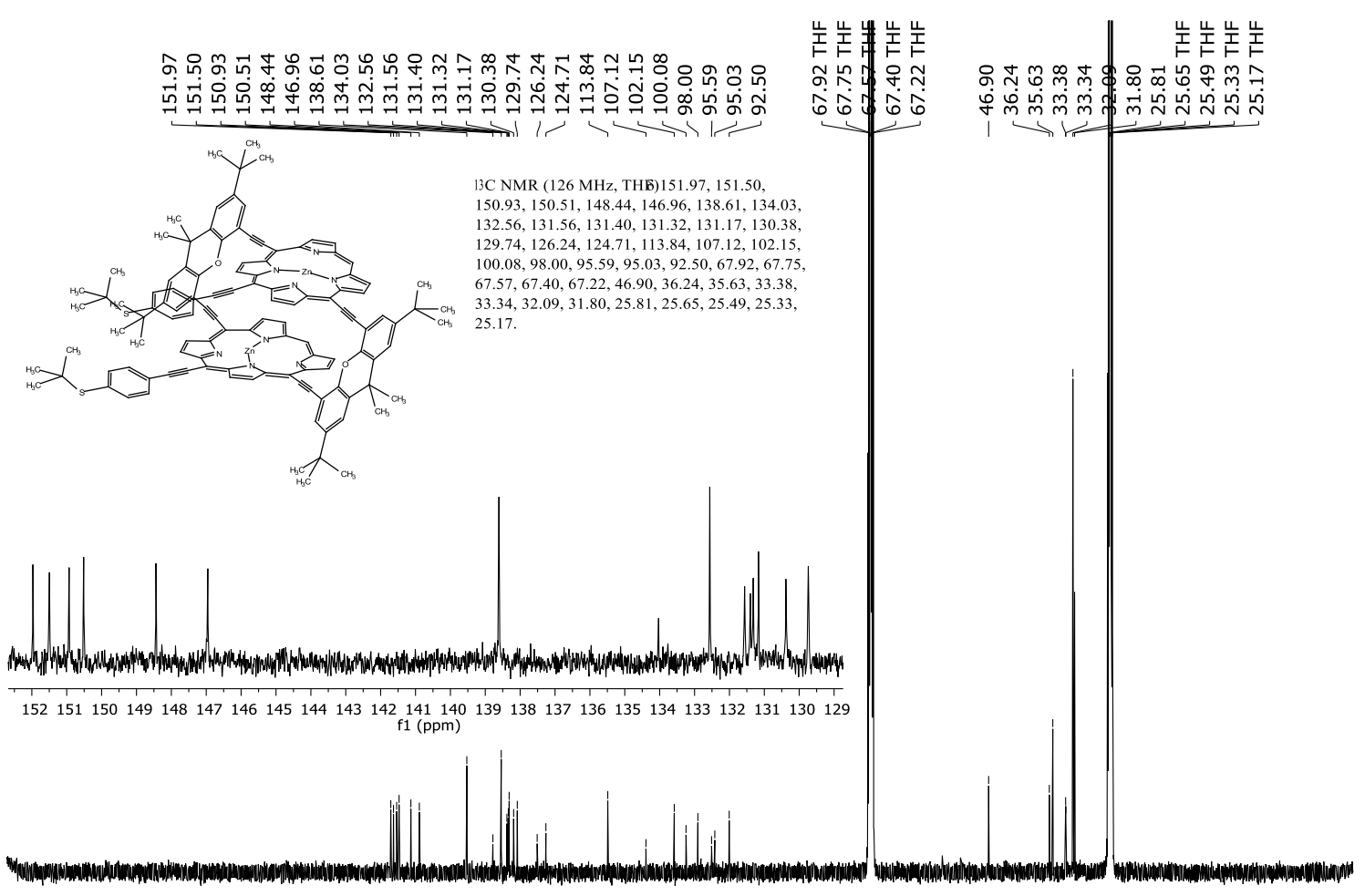

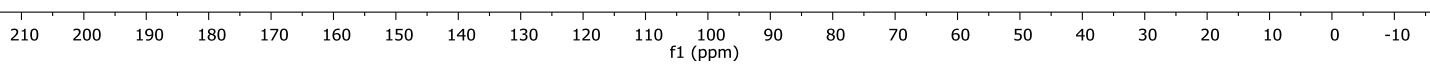

Figure $S 24:{ }^{13} \mathrm{C}\left\{{ }^{1} \mathrm{H}\right\}-N M R$ spectrum of 9. 


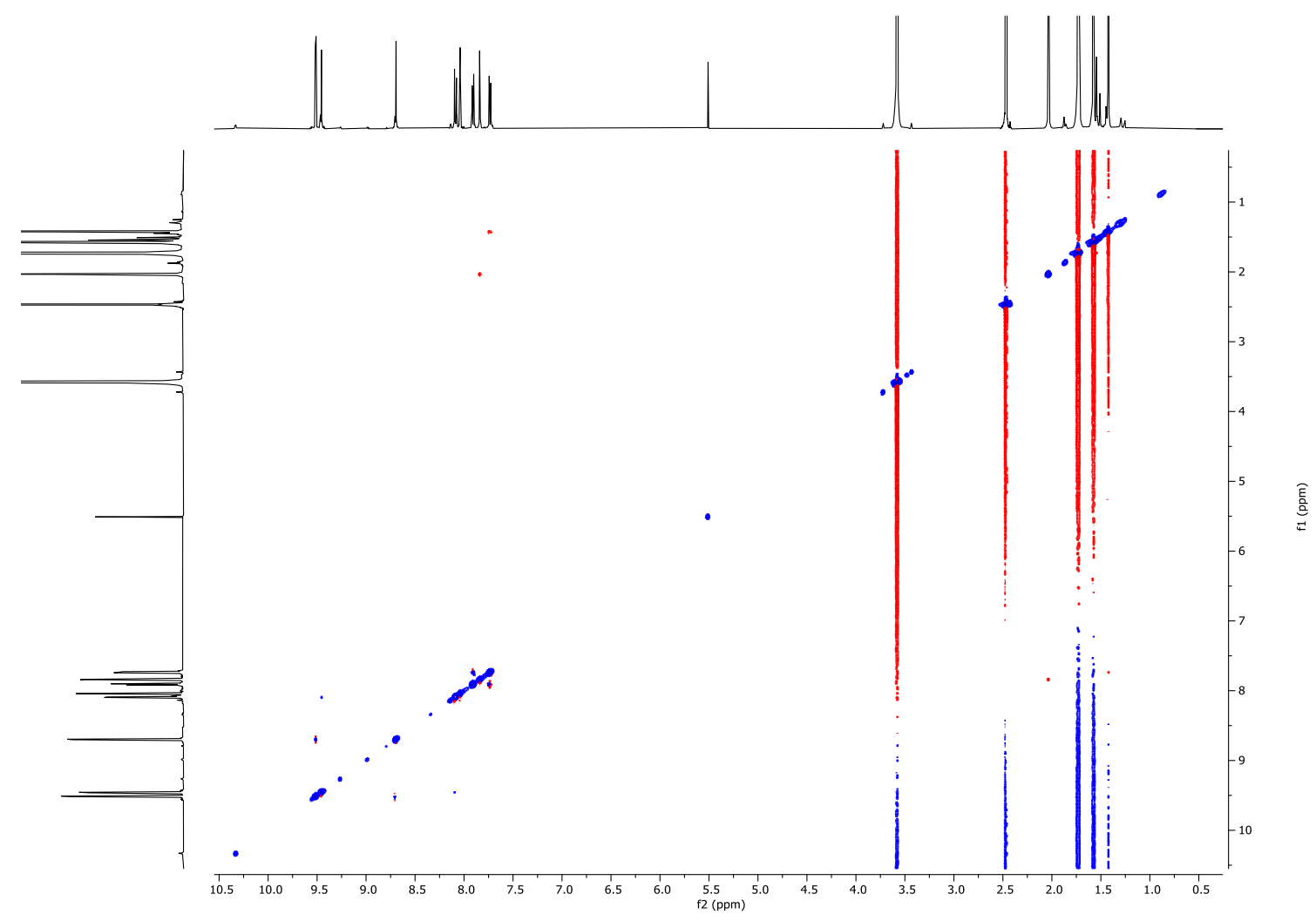

Figure $S$ 25: ${ }^{1} \mathbf{H}-{ }^{-1} \mathbf{H}$ NOESY spectrum of $\mathbf{9}$.

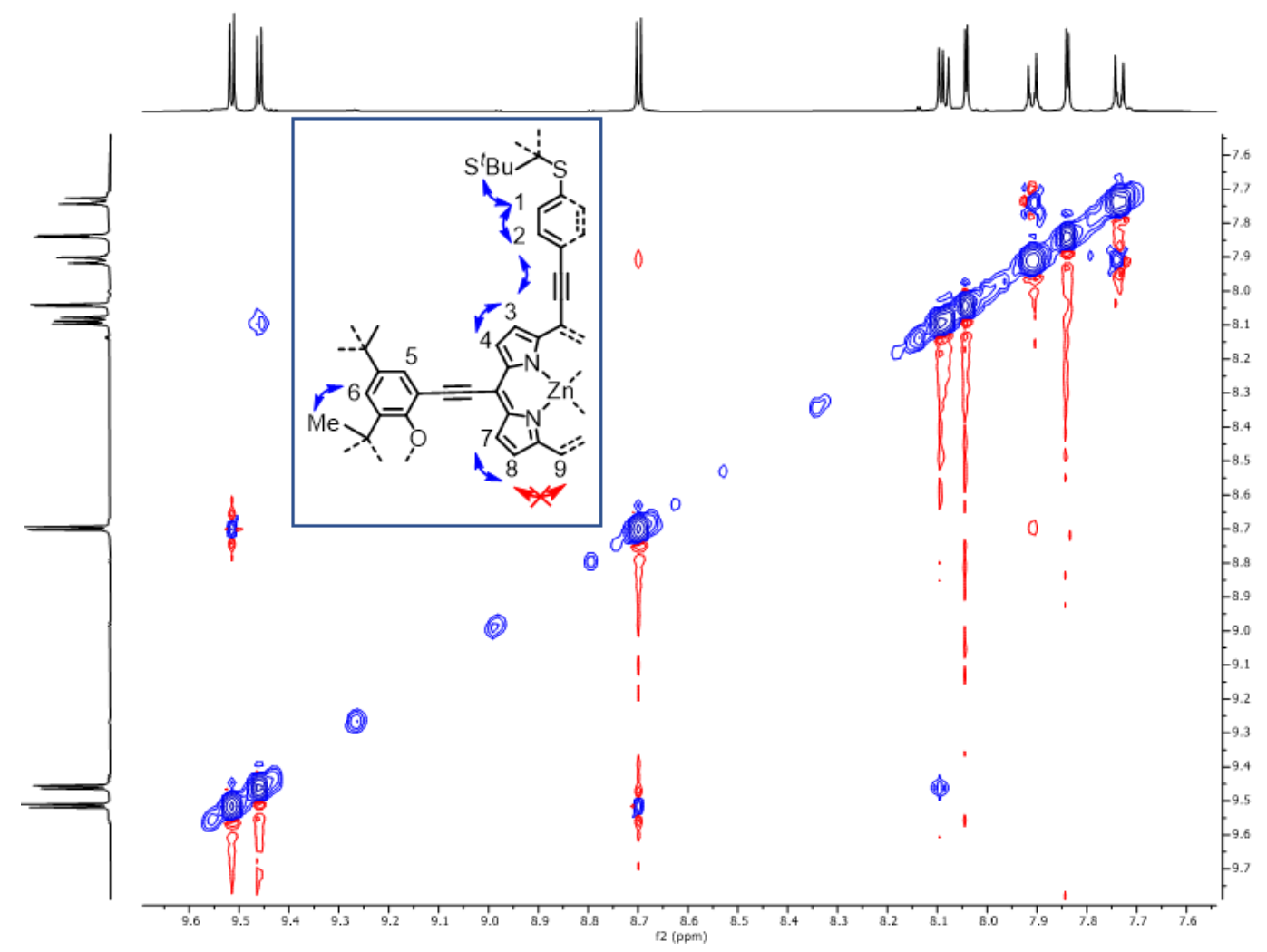

Figure S 26: Aromatic region of the ${ }^{1} \mathrm{H}^{-1} \mathrm{H}$ NOESY spectrum of 9. 


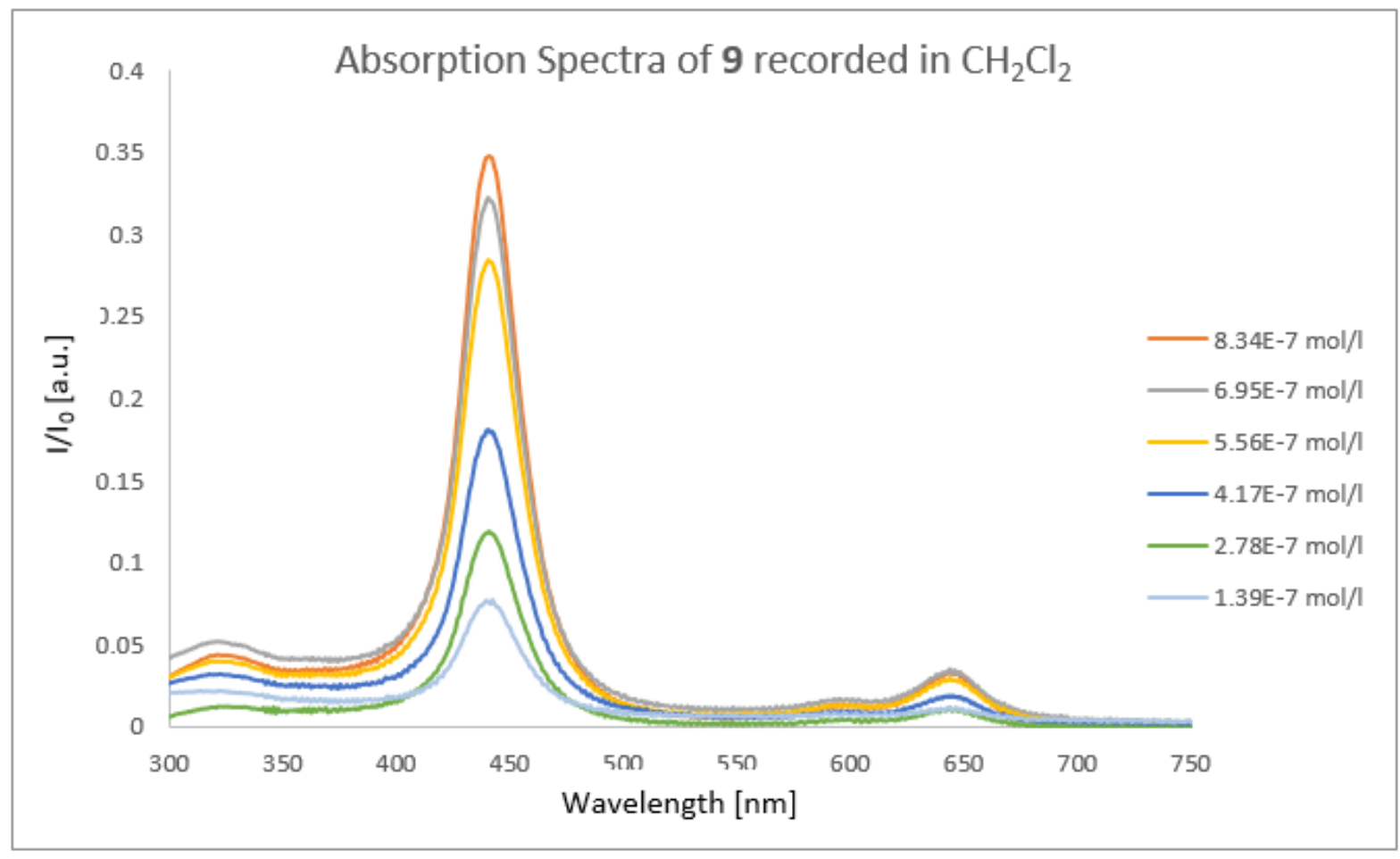

Figure S 27: Absorption spectra of a dilution series of 9 measured in $\mathrm{CH}_{2} \mathrm{Cl}_{2}$.

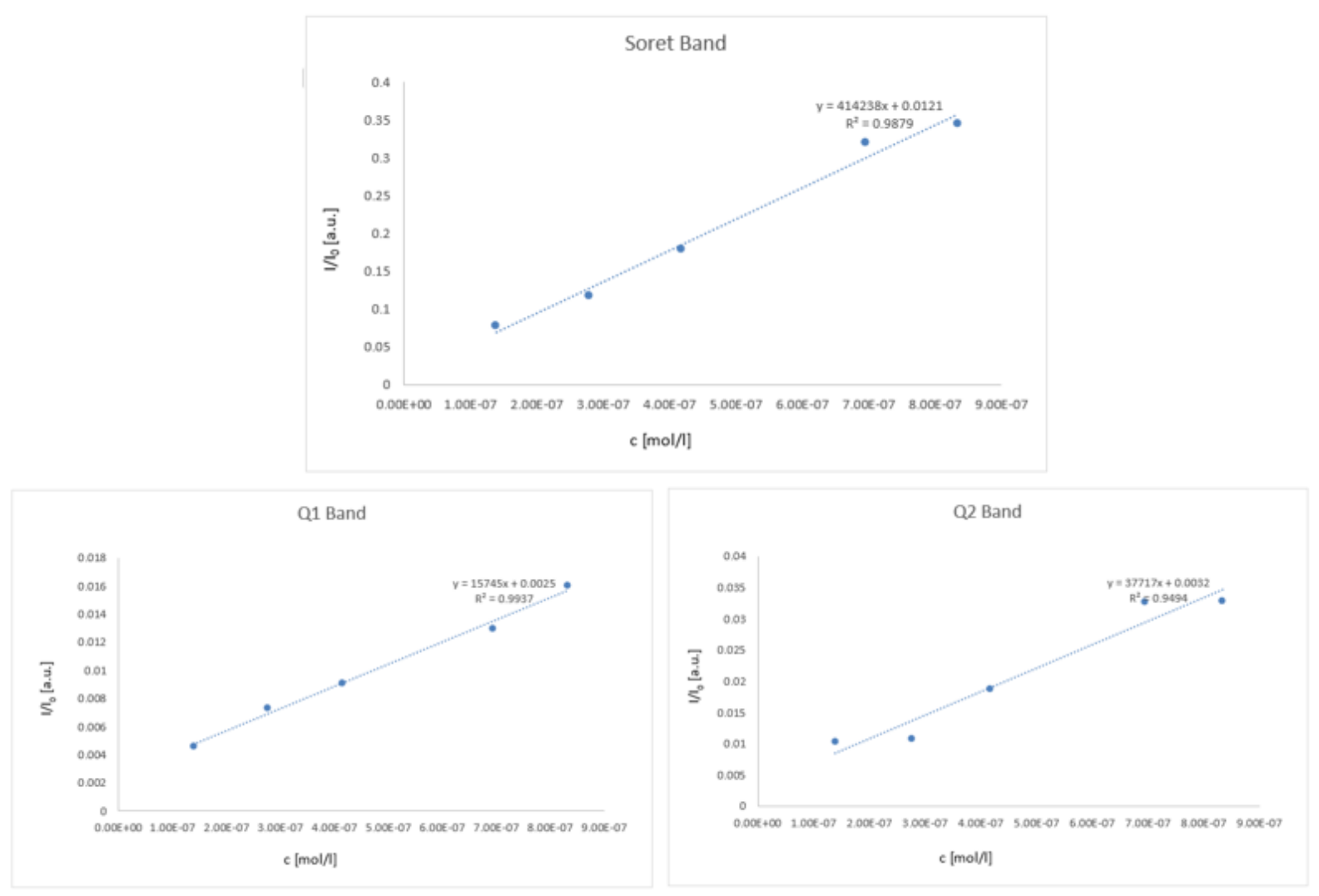

Figure S 28: Absorption versus concentration plots of 9 for the Soret, Q1, and Q2 bands. 


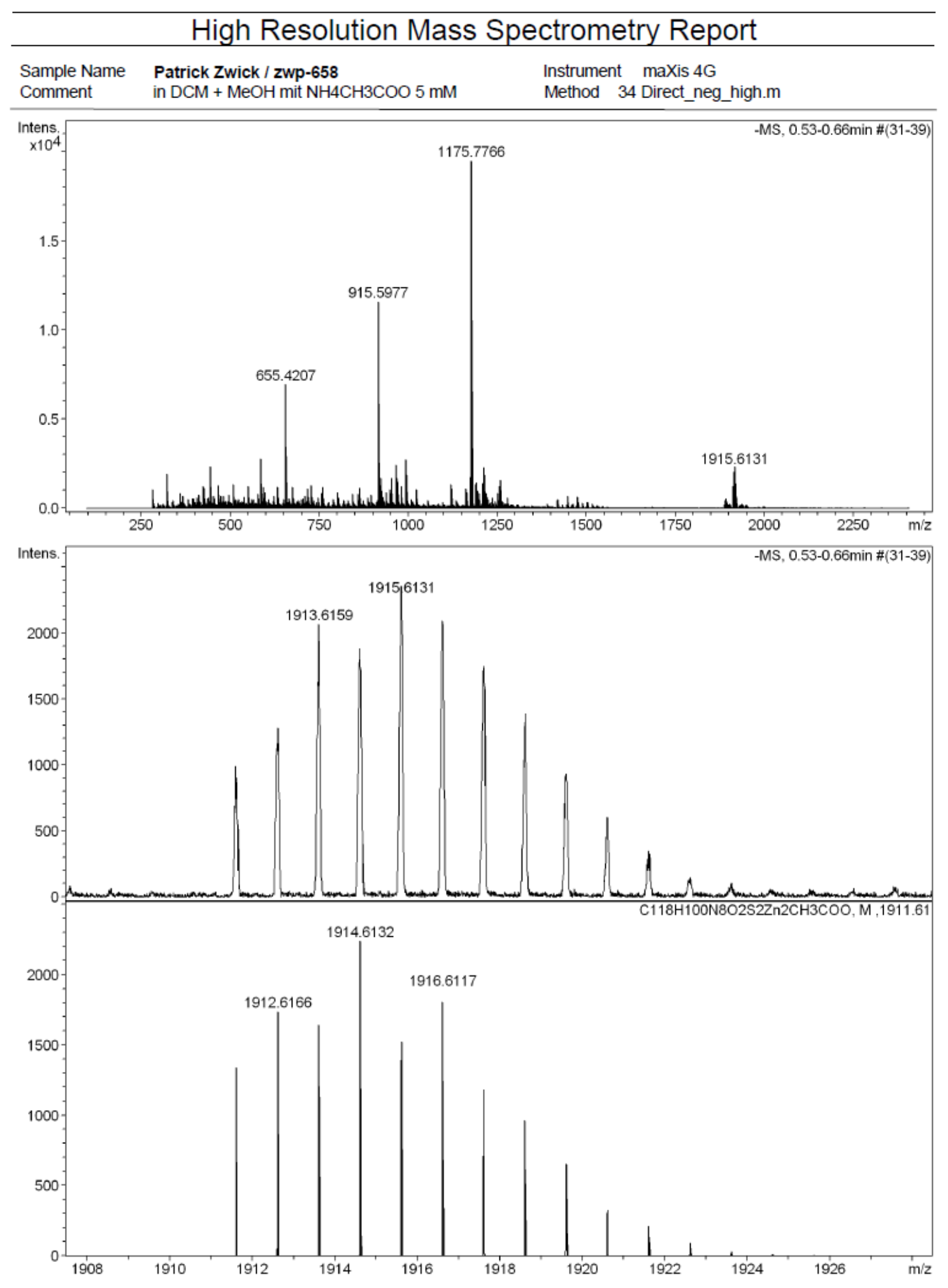

Figure S 29: HR-ESI MS of 9. 


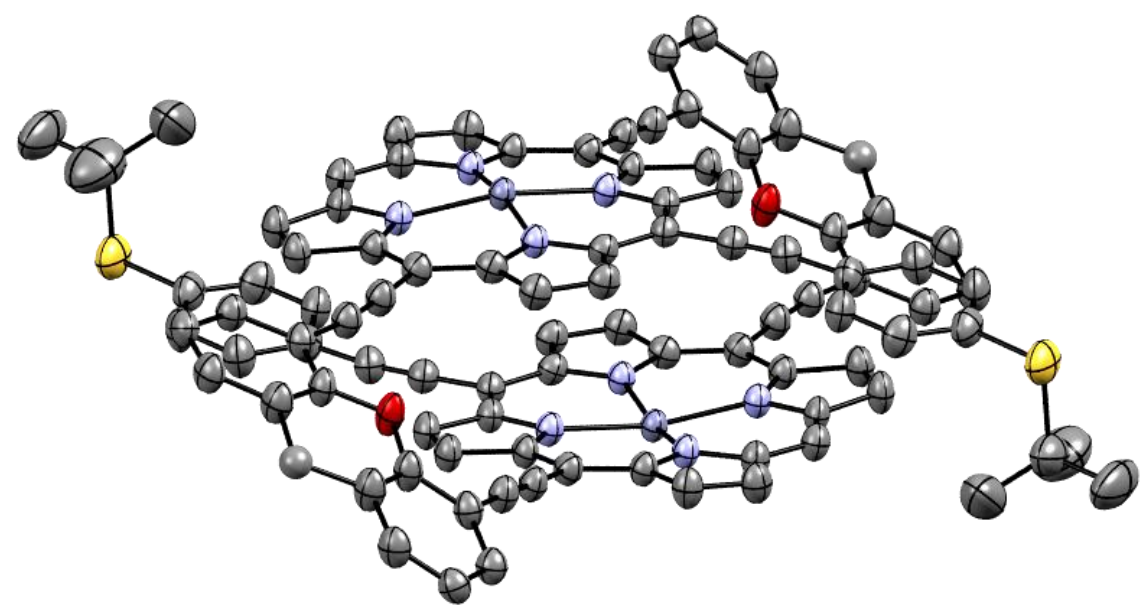

Figure S 30: ORTEP-representation of the solid state structure of 8 . Thermal ellipsoids are plotted on a $50 \%$ probability level. Solvent molecules and bulky groups are omitted for clarity. Single crystals suitable for $x$-ray analysis were obtained by slow diffusion of methanol into a tetrahydrofuran (THF) solution of 8 using the toplayering method. 
Table S2 Crystal data and structure refinement for $\mathbf{8}$.

$\begin{array}{ll}\text { Empirical formula } & \mathrm{C}_{142} \mathrm{H}_{146} \mathrm{~N}_{8} \mathrm{O}_{8} \mathrm{~S}_{2} \mathrm{Zn}_{2} \\ \text { Formula weight } & 2287.52 \\ \text { Temperature/K } & 180 \\ \text { Crystal system } & \text { triclinic } \\ \text { Space group } & P 1 \\ \mathrm{a} / \AA & 13.9372(16) \\ \mathrm{b} / \AA & 14.2654(12) \\ \mathrm{c} / \AA & 18.7976(14) \\ \alpha /{ }^{\circ} & 96.334(7) \\ \beta /{ }^{\circ} & 105.122(7) \\ \gamma /{ }^{\circ} & 107.575(7) \\ \text { Volume } / \AA^{3} & 3366.1(6) \\ \mathrm{Z} & 1 \\ \rho_{\text {calcg }} / \mathrm{cm}^{3} & 1.128 \\ \mu / \mathrm{mm}^{-1} & 0.737 \\ \mathrm{~F}(000) & 1210.0 \\ \mathrm{Crystal} \mathrm{size} / \mathrm{mm}^{3} & 0.18 \times 0.17 \times 0.16 \\ \text { Radiation } & \mathrm{GaK} \alpha(\lambda=1.34143)\end{array}$

$2 \Theta$ range for data collection $/{ }^{\circ} 4.328$ to 118

$\begin{array}{ll}\text { Index ranges } & -17 \leq \mathrm{h} \leq 14,-10 \leq \mathrm{k} \leq 18,-24 \leq 1 \leq 24 \\ \text { Reflections collected } & 40305 \\ \text { Independent reflections } & 14486\left[\mathrm{R}_{\text {int }}=0.0244, \mathrm{R}_{\text {sigma }}=0.0261\right] \\ \text { Indep. refl. with } \mathrm{I} \geq 2 \sigma(\mathrm{I}) & 11257 \\ \text { Data/restraints/parameters } & 14486 / 0 / 686 \\ \text { Goodness-of-fit on } \mathrm{F}^{2} & 1.141 \\ \text { Final R indexes [I } \geq 2 \sigma(\mathrm{I})] & \mathrm{R}_{1}=0.0788, \mathrm{wR}_{2}=0.2459 \\ \text { Final R indexes [all data] } & \mathrm{R}_{1}=0.0923, \mathrm{wR}_{2}=0.2567 \\ \text { Largest diff. peak/hole / e } \AA^{-3} 0.82 /-0.73 \\ \text { CCDC number } & 2019591\end{array}$




\section{checkCIF/PLATON report}

Structure factors have been supplied for datablock(s) zn-porph

THIS REPORT IS FOR GUIDANCE ONLY. IF USED AS PART OF A REVIEW PROCEDURE FOR PUBLICATION, IT SHOULD NOT REPLACE THE EXPERTISE OF AN EXPERIENCED CRYSTALLOGRAPHIC REFEREE.

No syntax errors found. CIF dictionary Interpreting this report

\section{Datablock: zn-porph}

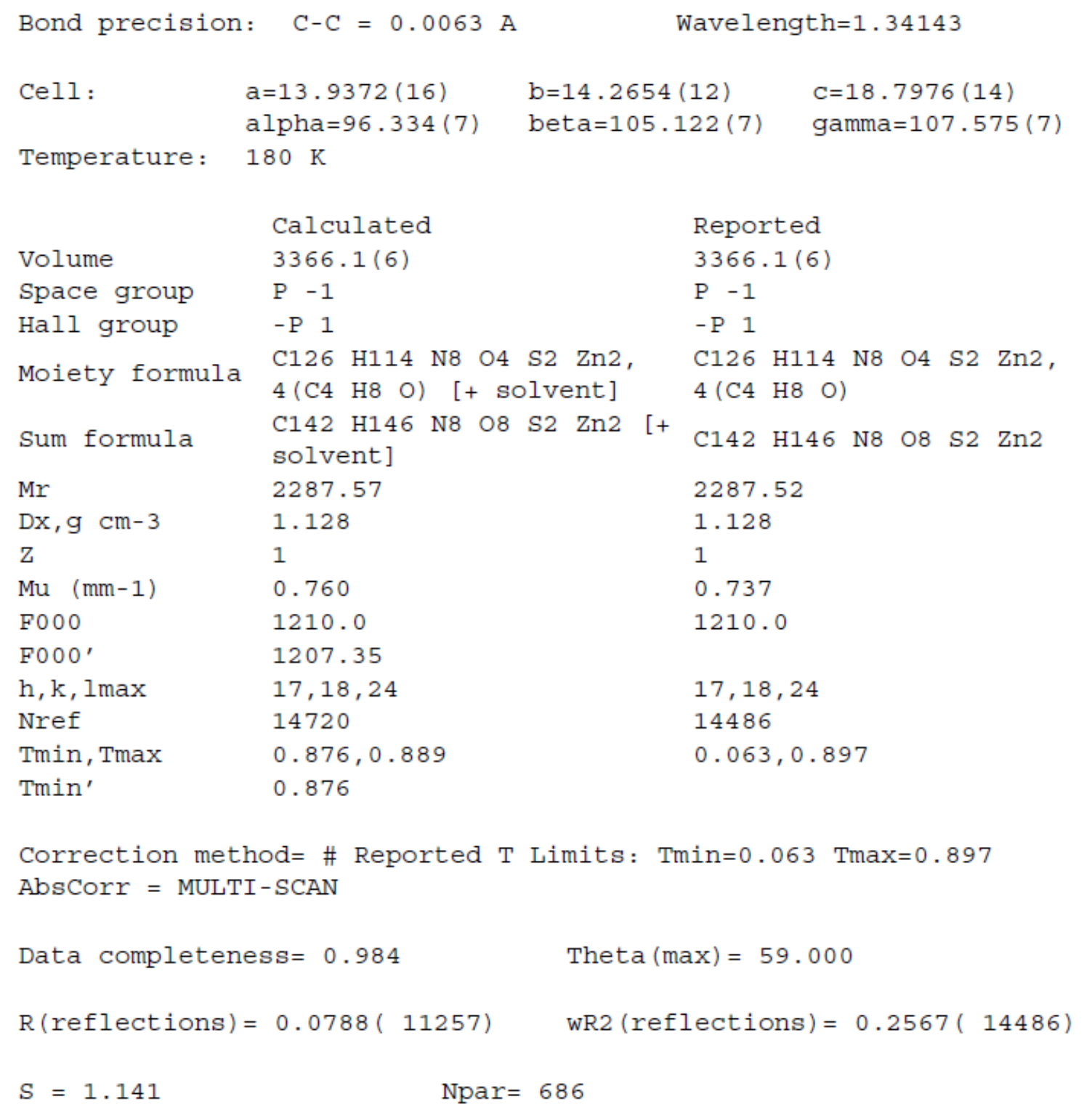

Figure S 31: Crystallographic data of 8. 


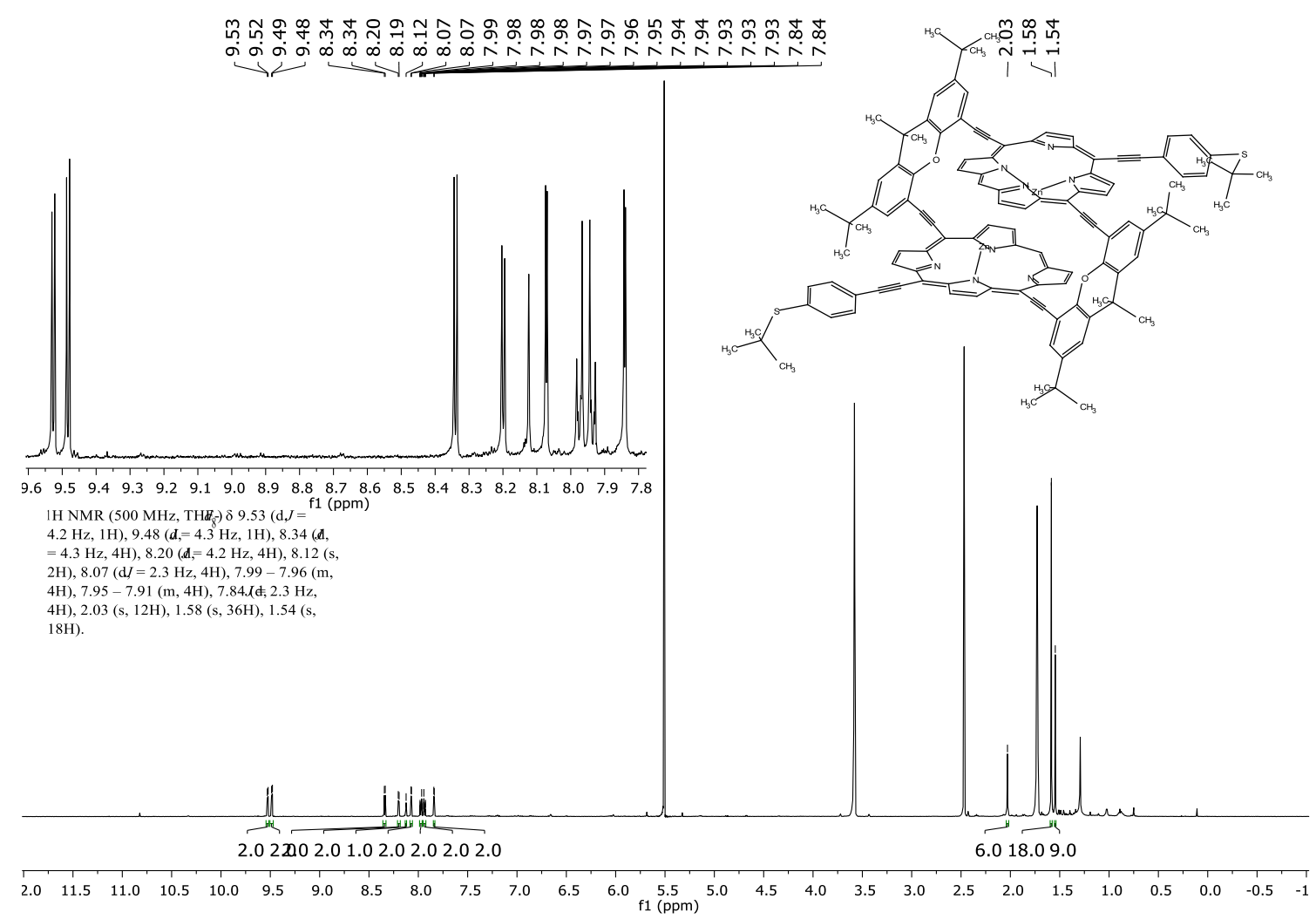

Figure $S$ 32: ${ }^{1} \mathbf{H}-\mathrm{NMR}$ spectrum of 8.

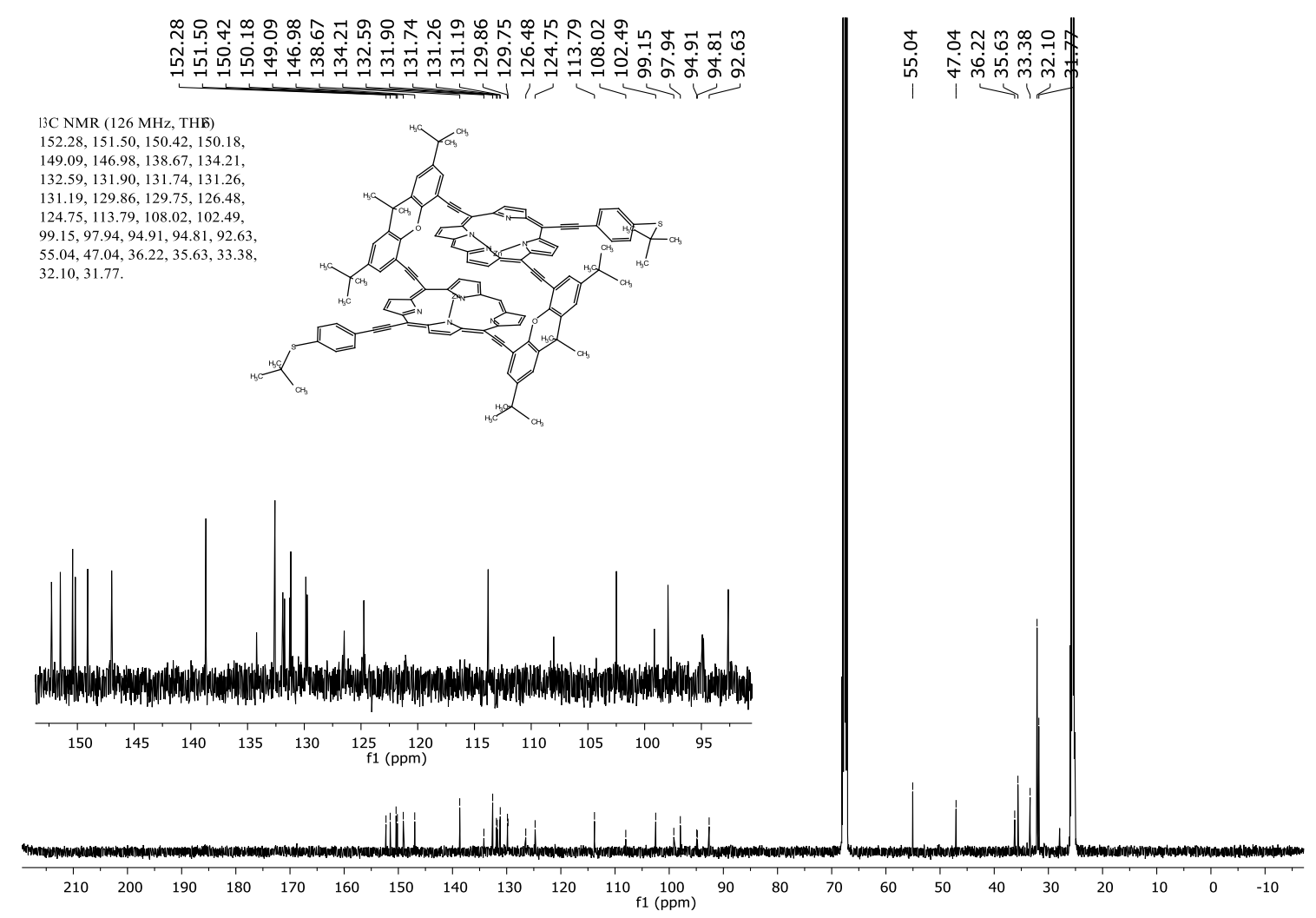

Figure $S 33:{ }^{13} \mathbf{C}\left\{{ }^{1} \mathbf{H}\right\}-N M R$ spectrum of 8 . 


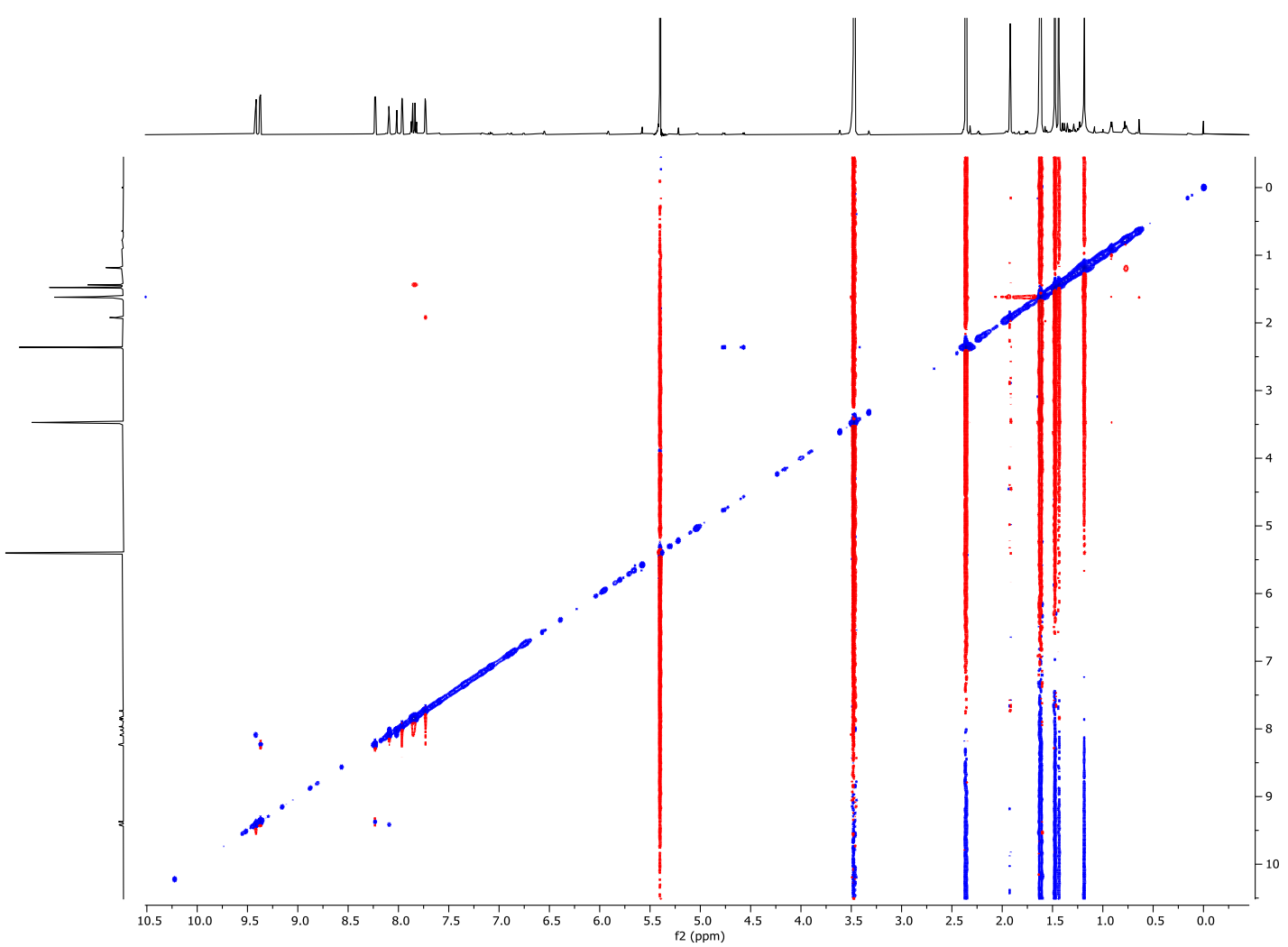

Figure S 34: ${ }^{1} \mathbf{H}-{ }^{1} \mathrm{H}$ NOESY spectrum of 8.

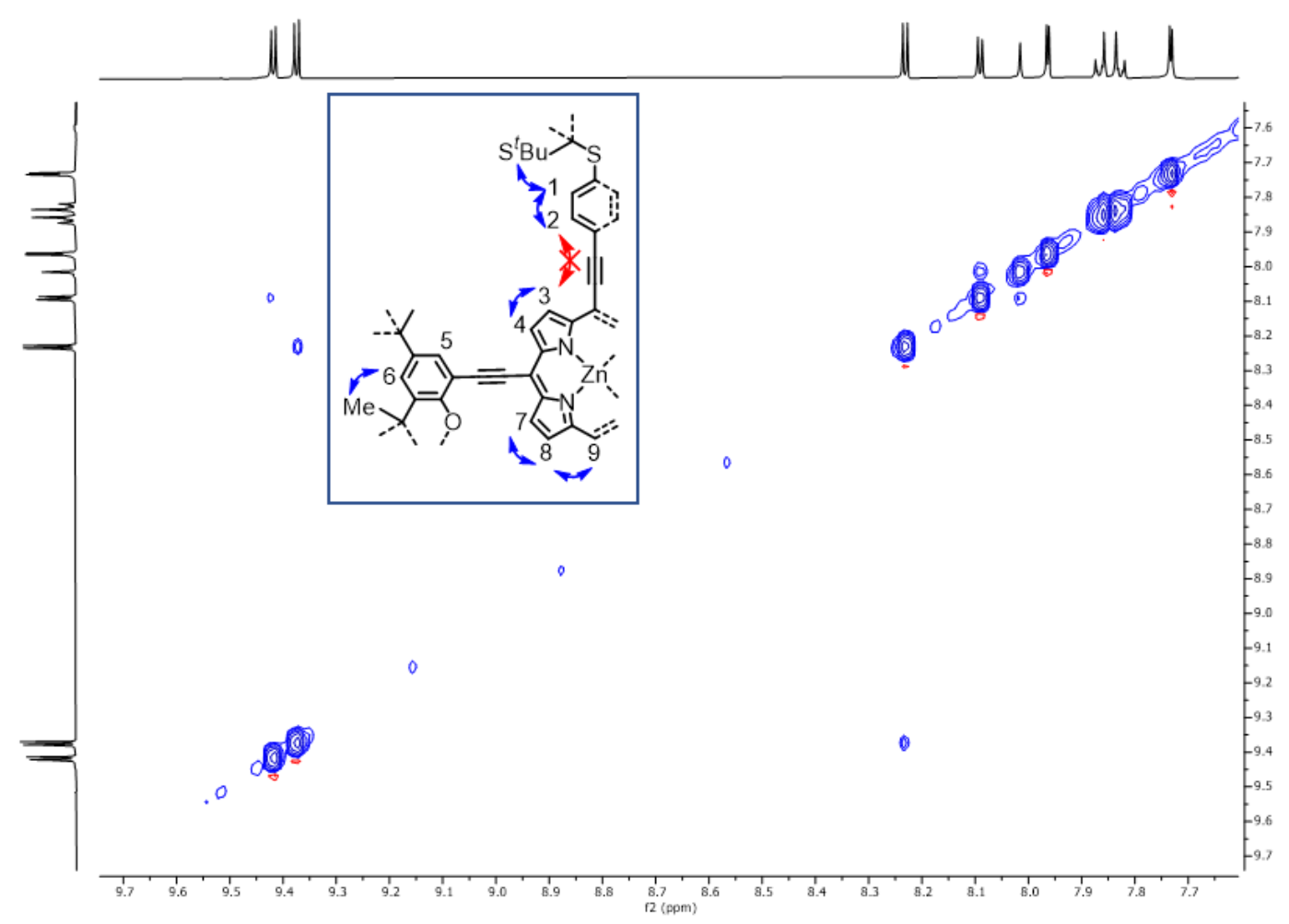

Figure S 35: Aromatic region of the ${ }^{1} \mathrm{H}-{ }^{1} \mathrm{H}$ NOESY spectrum of 8. 


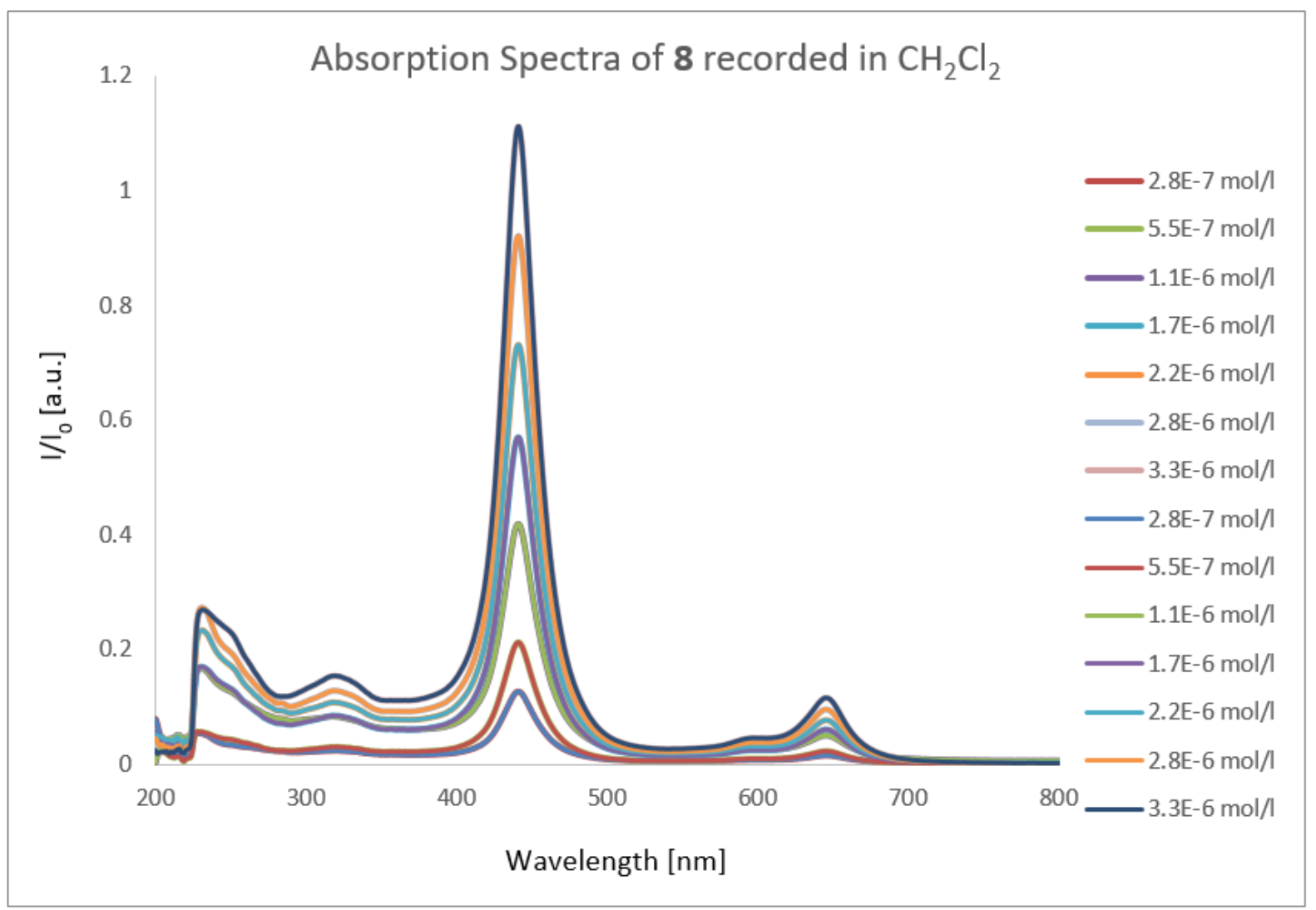

Figure S 36: Absorption spectra of a dilution series of 8 measured in $\mathrm{CH}_{2} \mathrm{Cl}_{2}$.
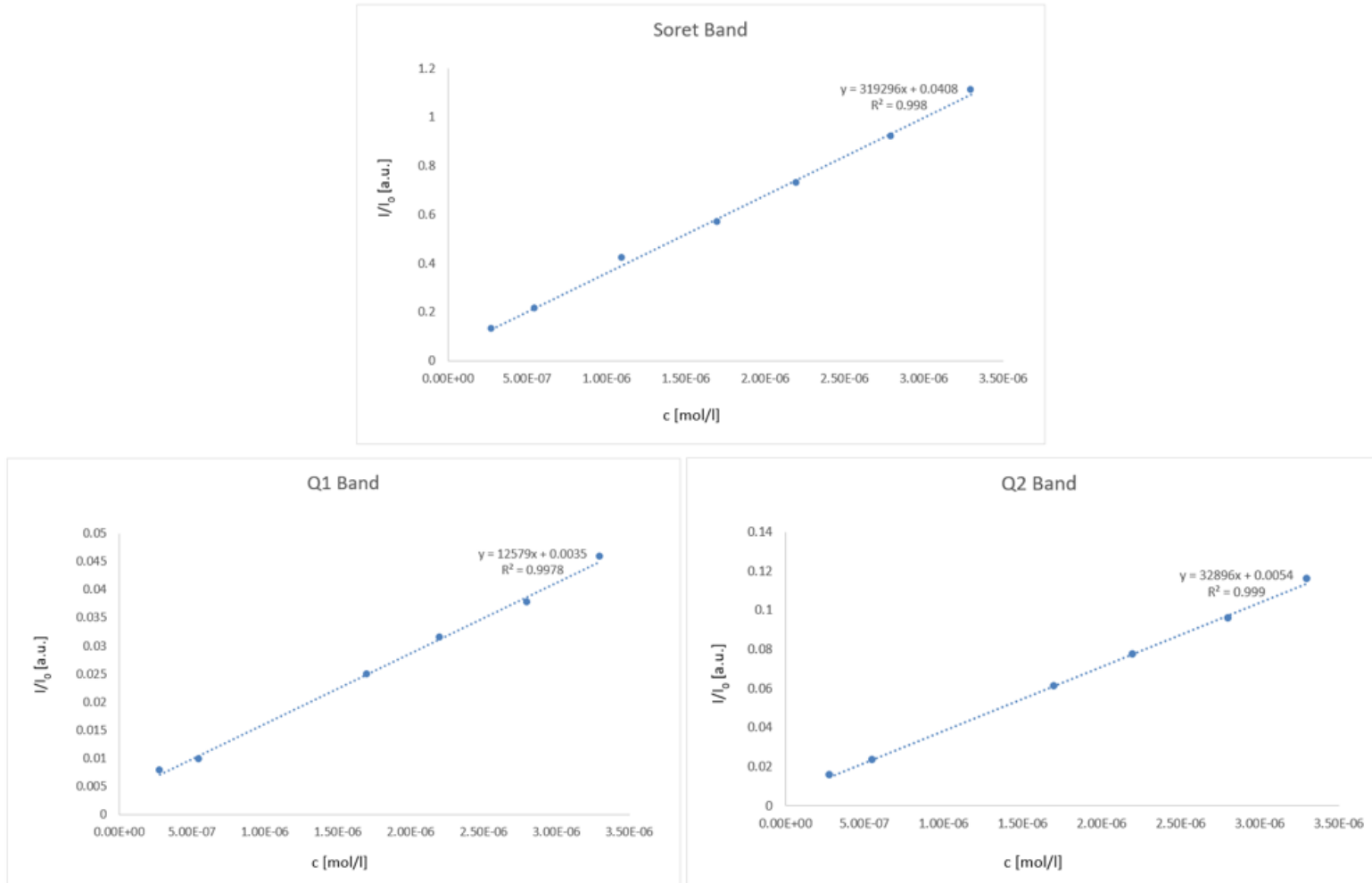

Figure S 37: Absorption versus concentration plots of 8 for the Soret, Q1, and Q2 bands. 


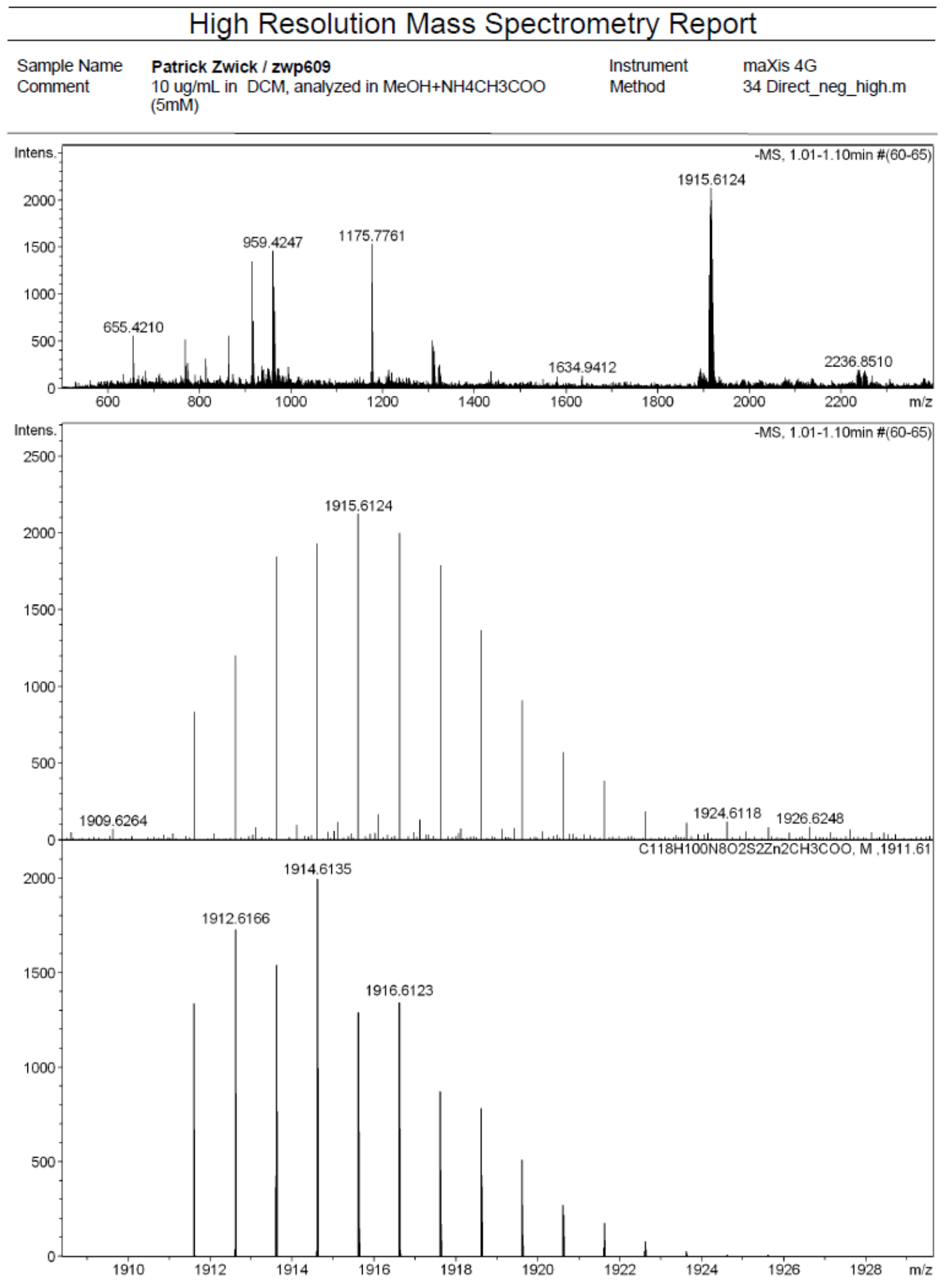

Figure S 38: HR-ESI MS of 8. 


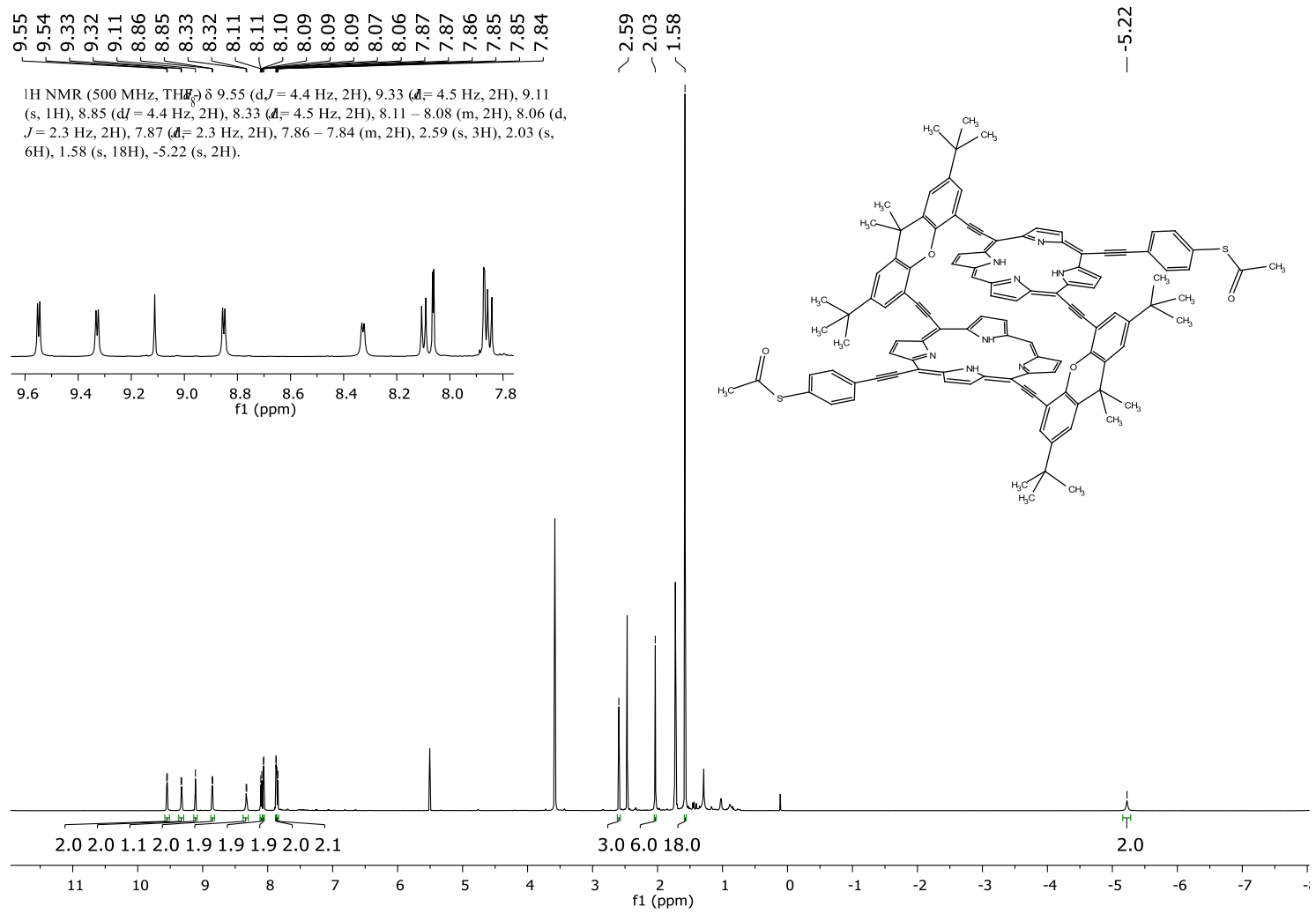

Figure $S$ 39: ${ }^{1} \mathrm{H}-\mathrm{NMR}$ spectrum of $\mathbf{1 0}$.

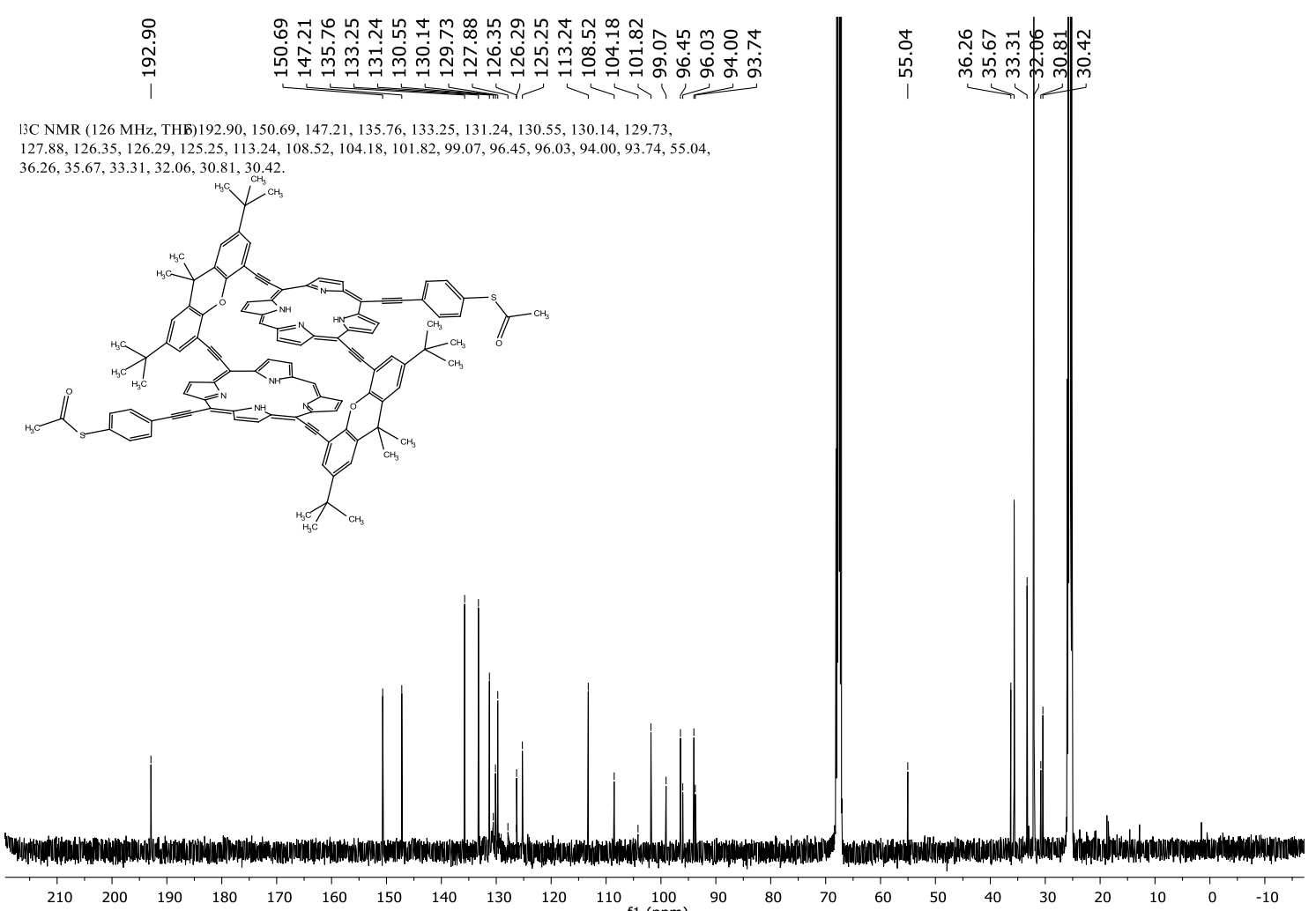

Figure $S 40:{ }^{13} \mathbf{C}\left\{{ }^{1} \mathrm{H}\right\}-N M R$ spectrum of 10 . 


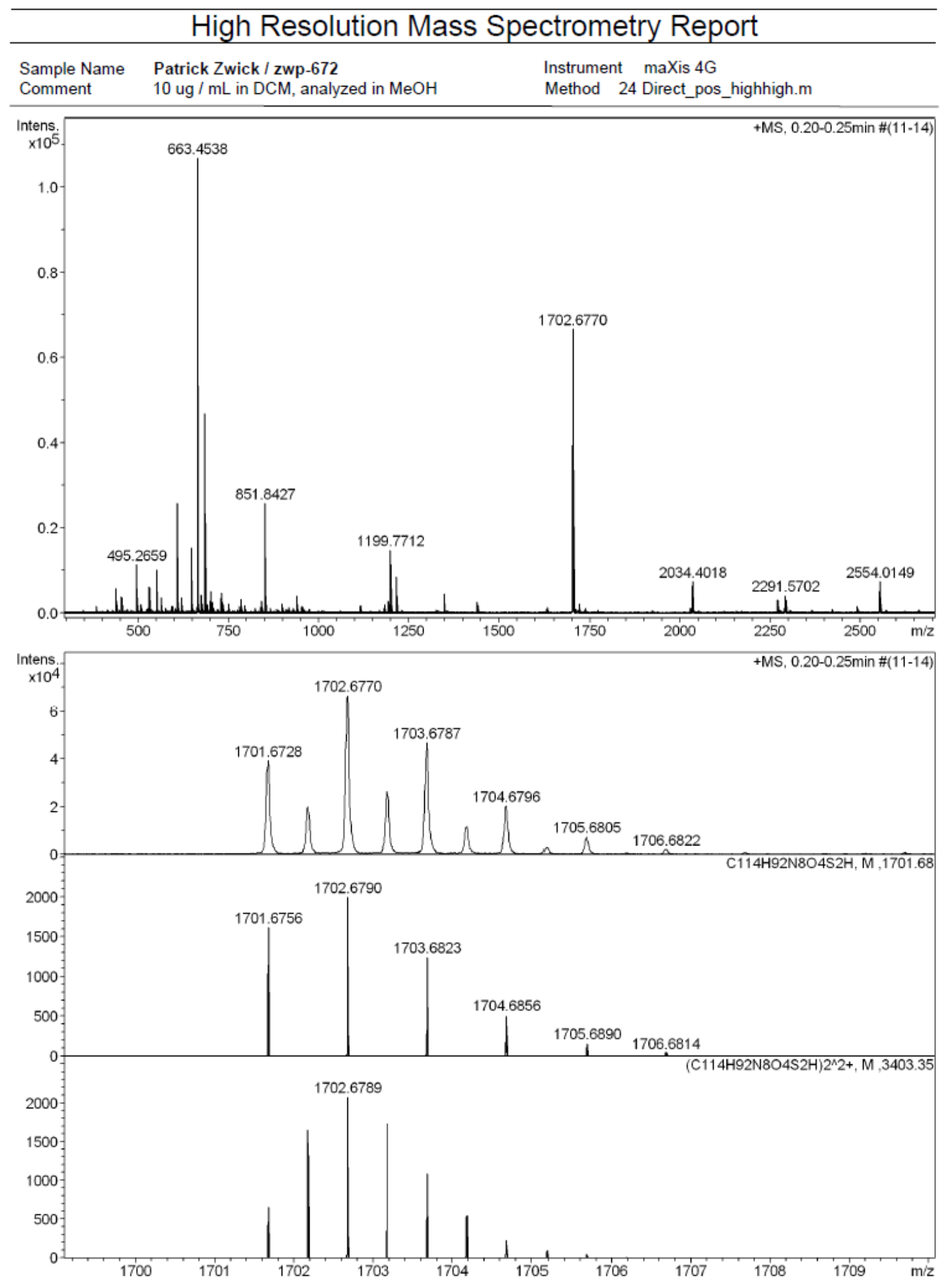

Figure S 41: HR-ESI MS of 10. 


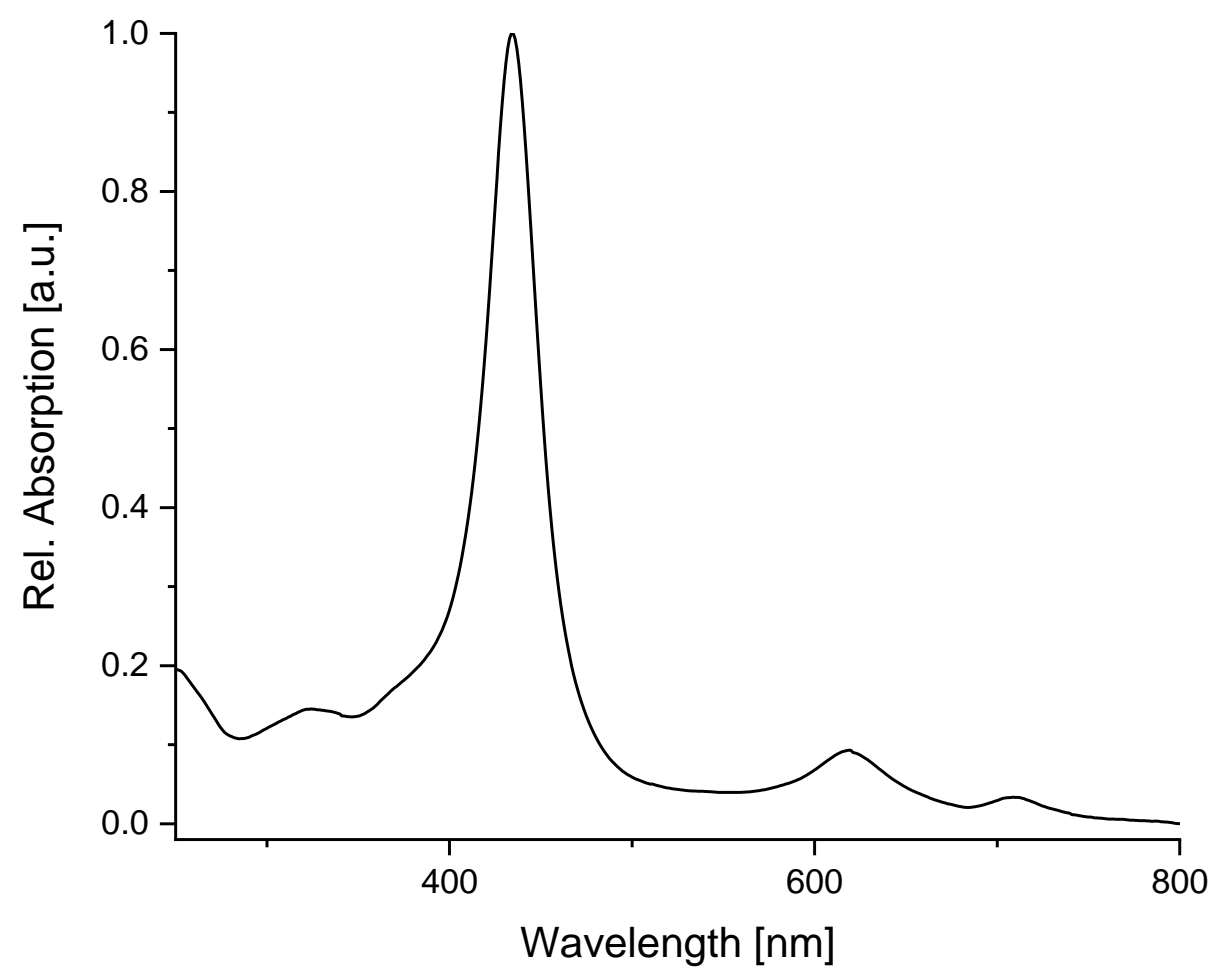

Figure $S$ 42: Absorption spectrum of 10 measured in $\mathrm{CH}_{2} \mathrm{Cl}_{2}$. 


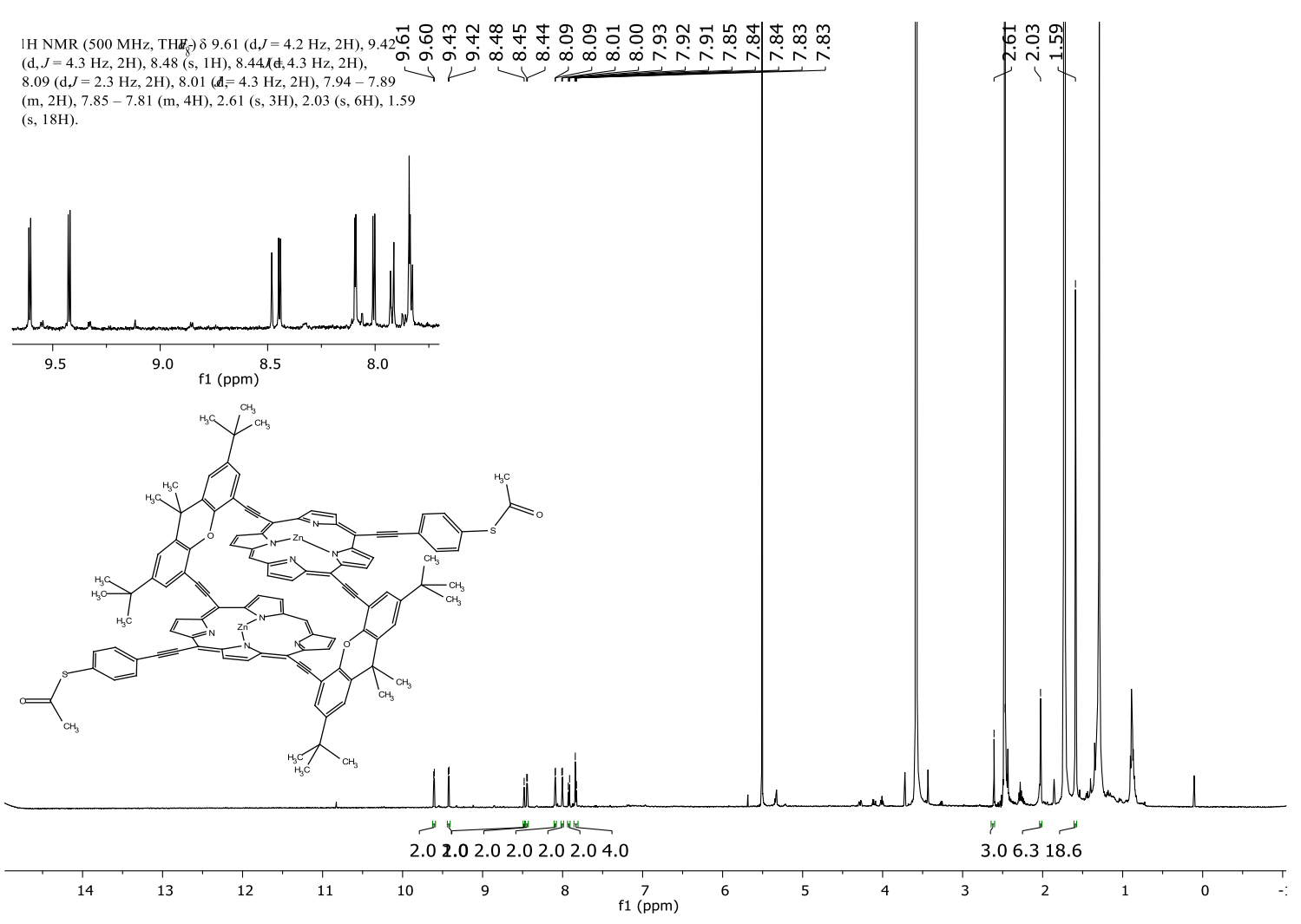

Figure $S$ 43: ${ }^{1} \mathbf{H}-\mathrm{NMR}$ spectrum of $\mathbf{1}$.

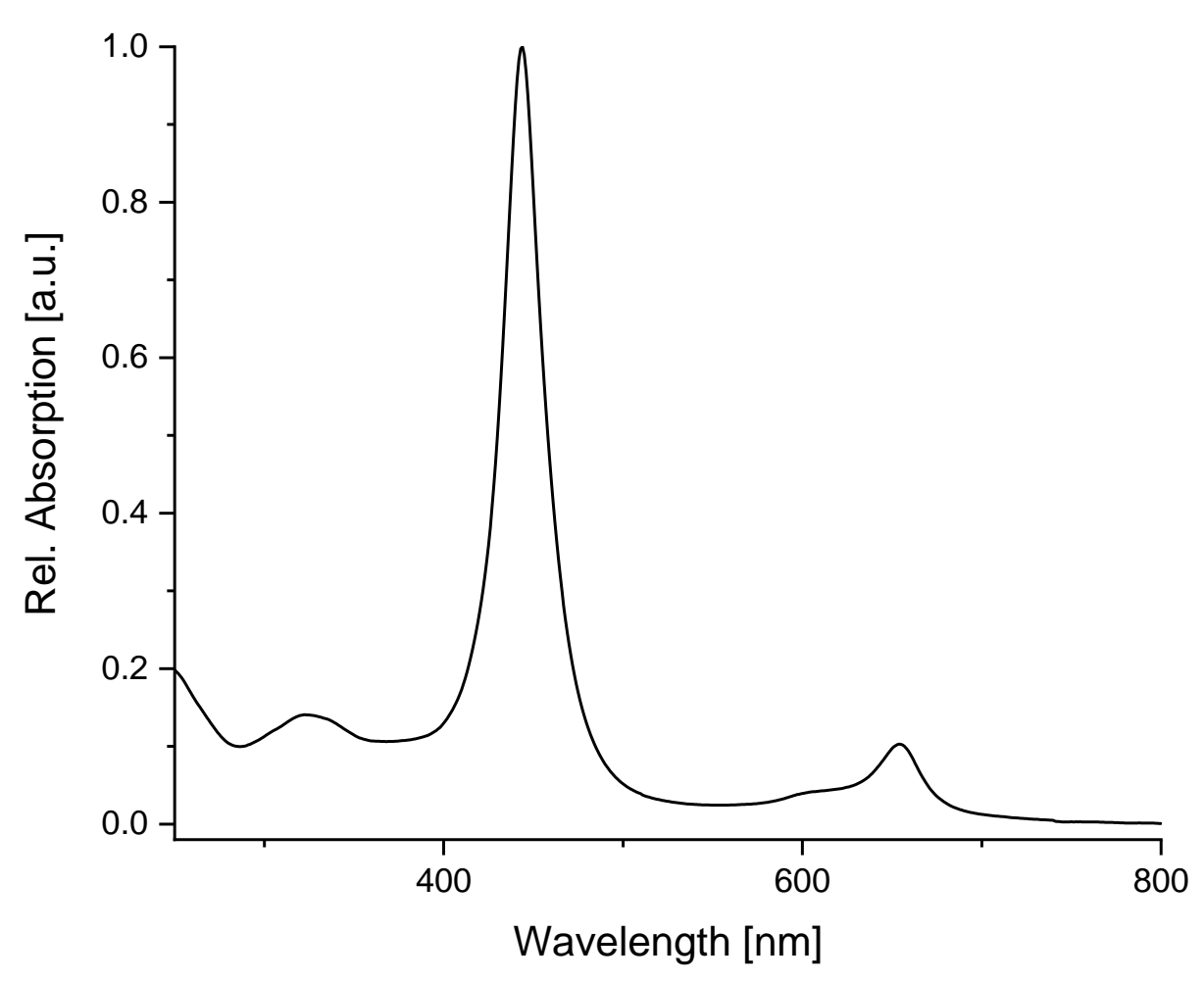

Figure S 44: Absorption spectrum of 1 measured in $\mathrm{CH}_{2} \mathrm{Cl}_{2}$. 


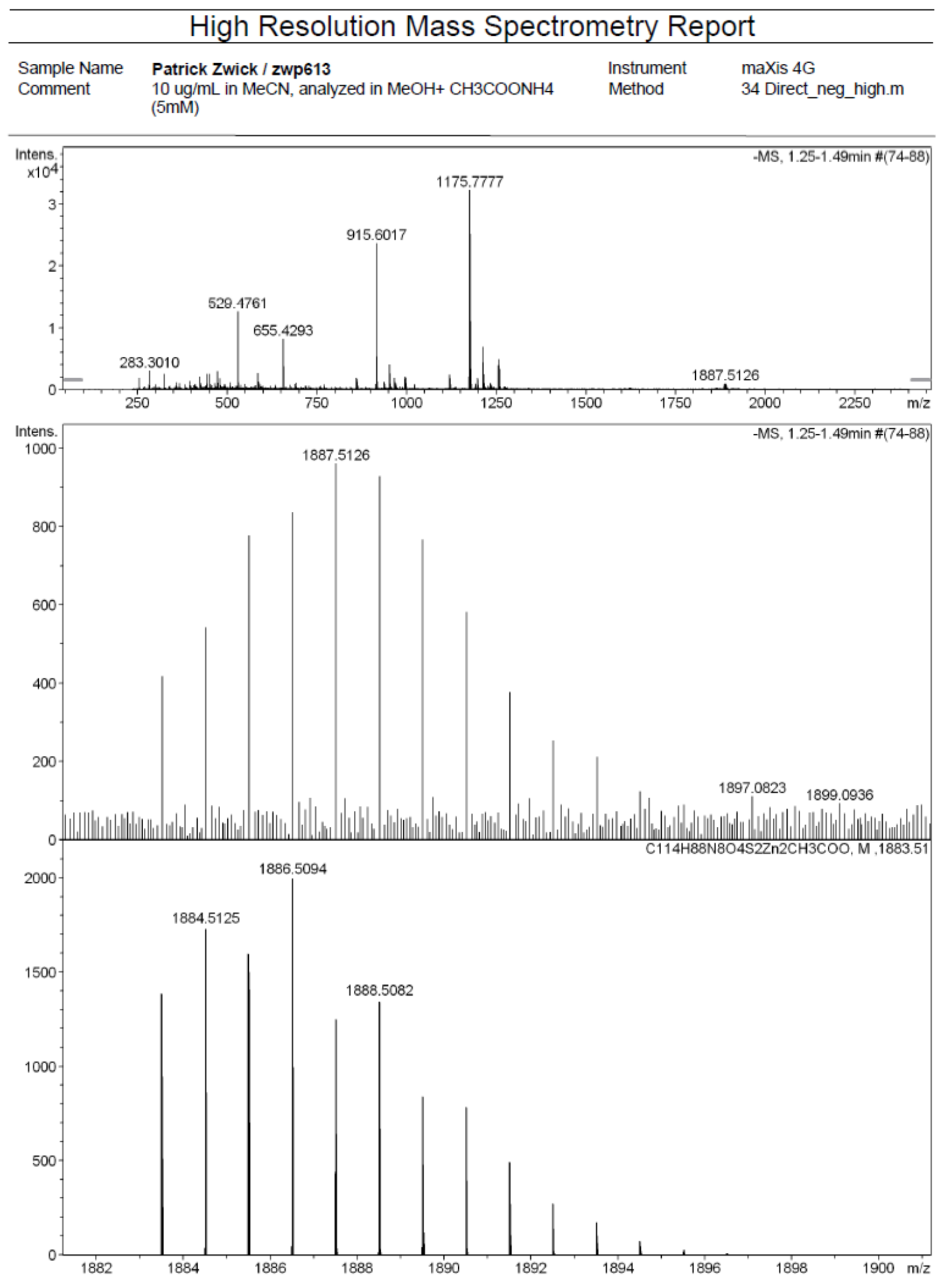

Figure S 45: HR-ESI MS of 1. 
IH NMR ( $\left.500 \mathrm{MHz}, \mathrm{TH} \mathbb{d}_{8}\right) \delta 9.45(\mathrm{~d}, J=4.5 \mathrm{~Hz}, 2 \mathrm{H}), 9.37(\mathrm{~d},=4.4 \mathrm{~Hz}, 2 \mathrm{H}), 9.09(\mathrm{~d},-$

$4.5 \mathrm{~Hz}, 2 \mathrm{H}), 8.62(\mathrm{~s}, 1 \mathrm{H}), 8.48(\notin \neq 4.4 \mathrm{~Hz}, 2 \mathrm{H}), 8.10-8.07(\mathrm{~m}, 2 \mathrm{H}), 8.01+\mathrm{ta} 2.3 \mathrm{~Hz}$,

$2 \mathrm{H}), 7.87(\mathrm{~d} J=2.3 \mathrm{~Hz}, 2 \mathrm{H}), 7.67-7.63(\mathrm{~m}, 2 \mathrm{H}), 2.54(\mathrm{~s}, 3 \mathrm{H}), 2.05(\mathrm{~s}, 3 \mathrm{H}), 2.04(\mathrm{~s}, 3 \mathrm{H}) 1.56$

$(\mathrm{s}, 18 \mathrm{H}),-5.72(\mathrm{~s}, 2 \mathrm{H})$

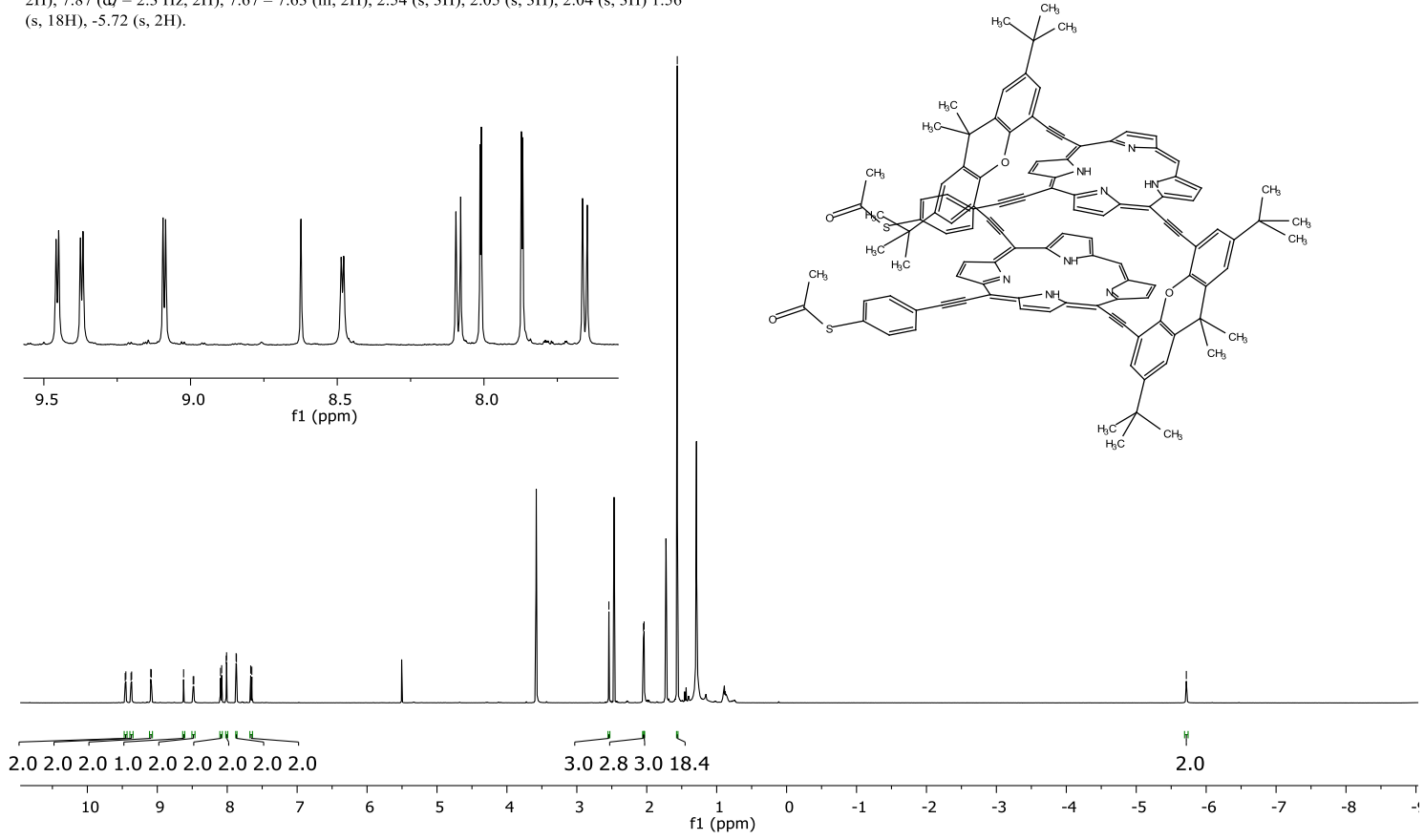

Figure $S$ 46: ${ }^{\mathbf{1}} \mathrm{H}-\mathrm{NMR}$ spectrum of $\mathbf{1 1 .}$

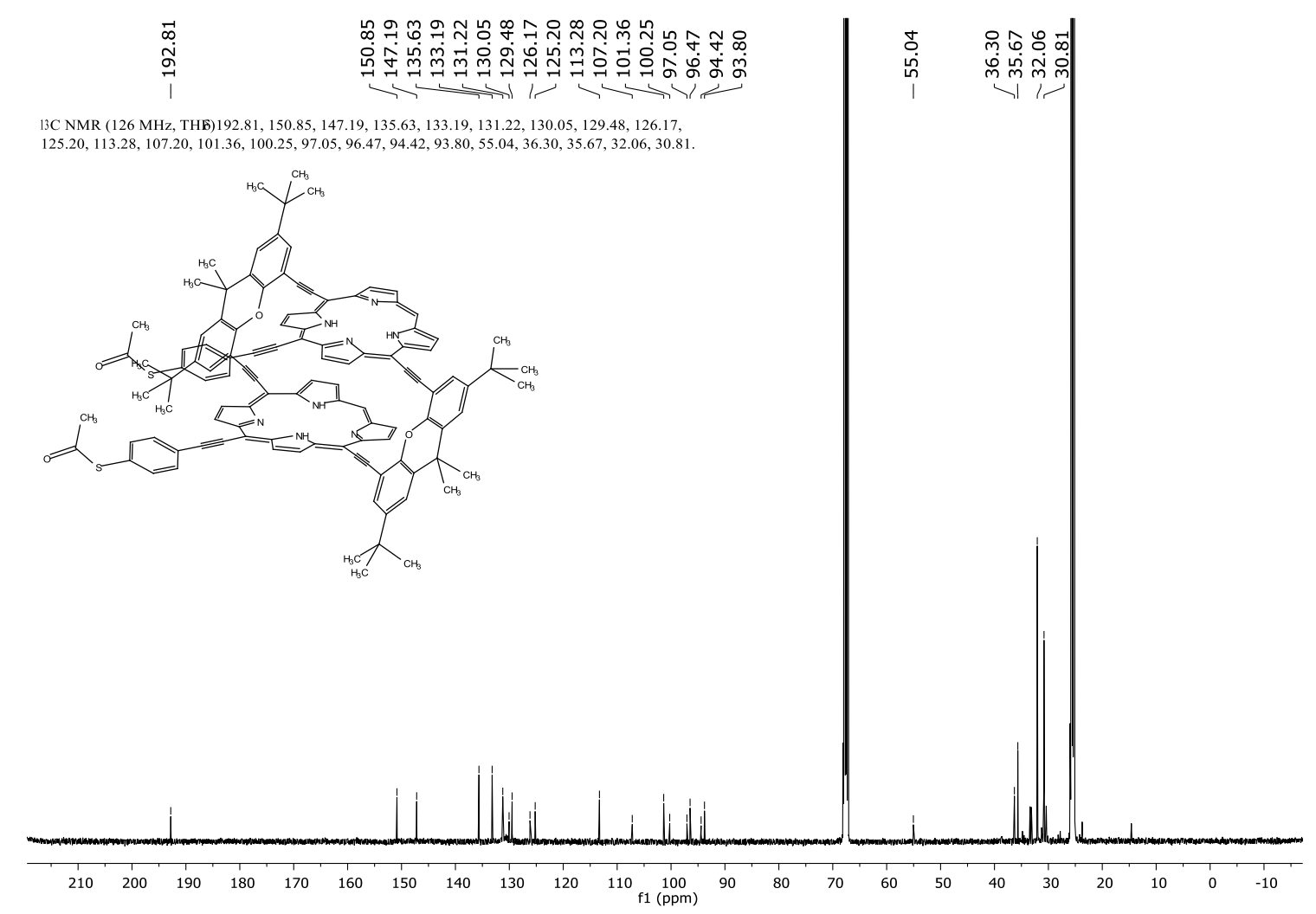

Figure $S 47:{ }^{13} \mathrm{C}\left\{{ }^{1} \mathrm{H}\right\}-N M R$ spectrum of 11. 


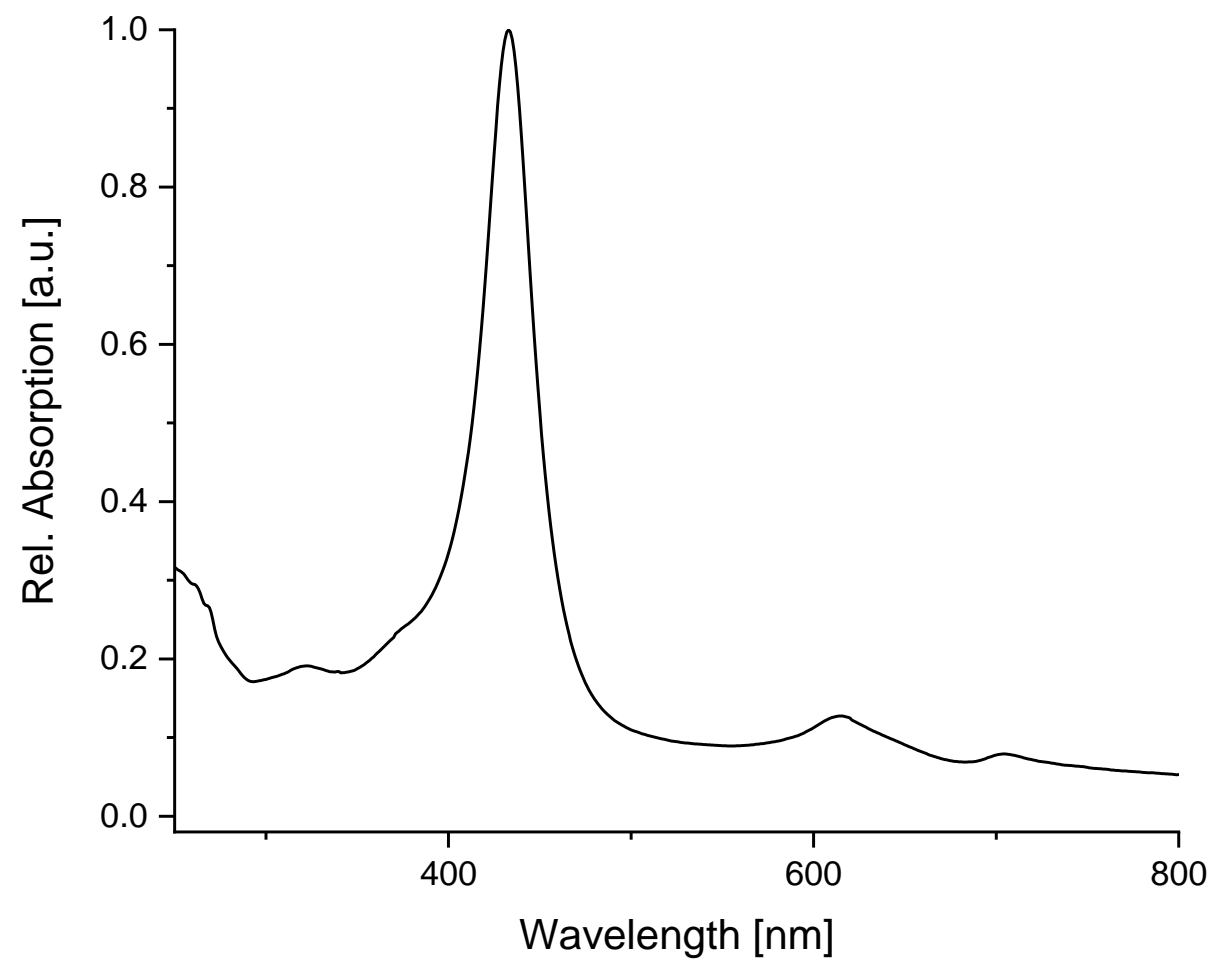

Figure S 48: Absorption spectrum of 11 measured in $\mathrm{CH}_{2} \mathrm{Cl}_{2}$. 


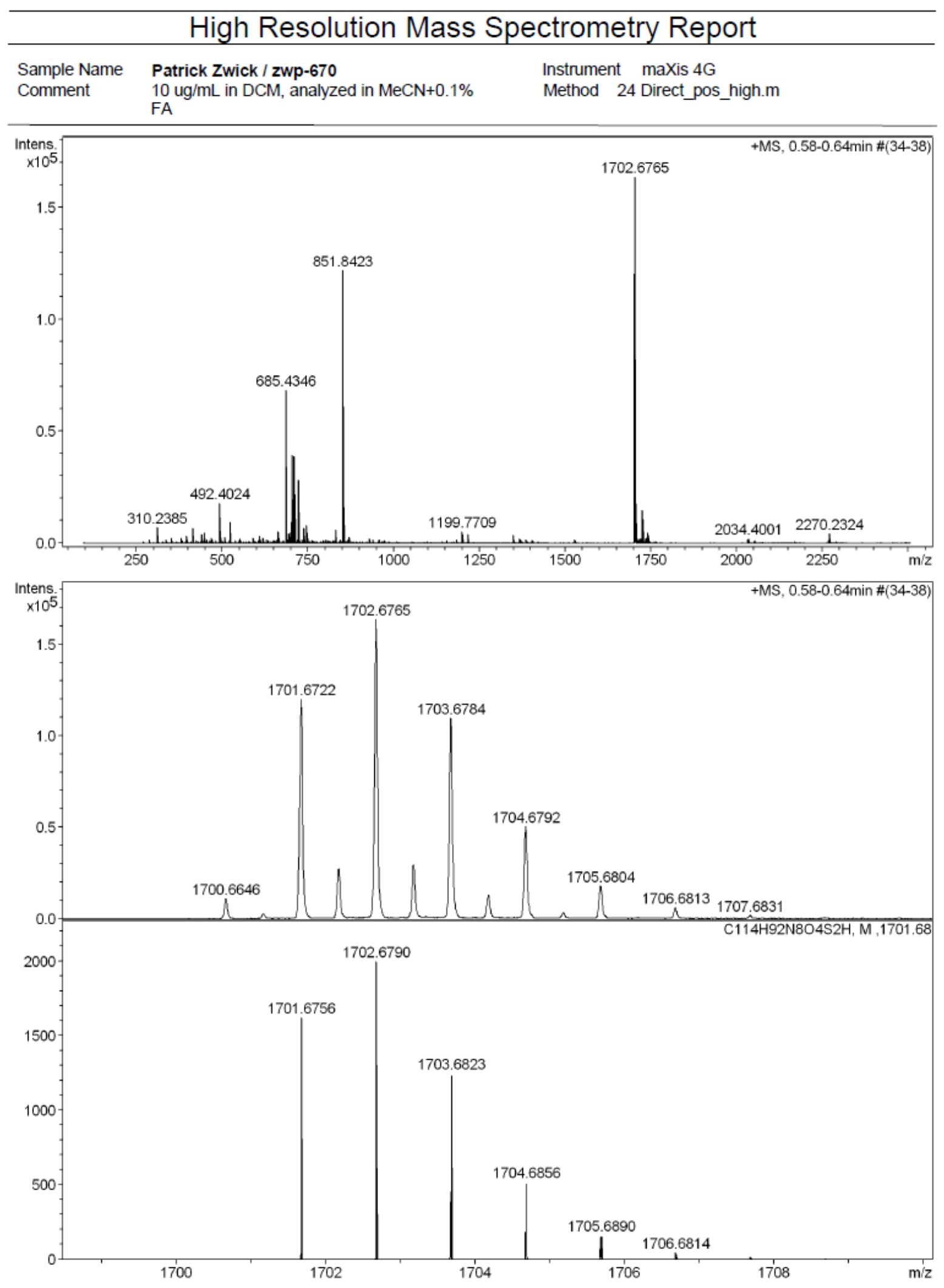

Figure S 49: HR-ESI MS of 11. 
IH NMR ( $\left.500 \mathrm{MHz}, \mathrm{TH} \boldsymbol{d}_{\bar{\gamma}}\right) 89.52(\mathrm{~d}, J=4.3 \mathrm{~Hz}, 2 \mathrm{H}), 9.46(\mathrm{~d}=4.2 \mathrm{~Hz}, 2 \mathrm{H}), 8.72(\mathrm{~d},=$

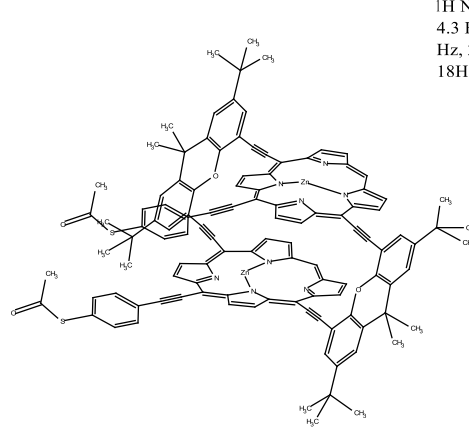

.

$.85(\mathrm{~d},=2.3 \mathrm{~Hz}, 2 \mathrm{H}), 7.62(\mathrm{~d}=8.1 \mathrm{~Hz}, 2 \mathrm{H}), 2.51(\mathrm{~s}, 3 \mathrm{H}), 2.04(\mathrm{~s}, 6 \mathrm{H}), 1.58(\mathrm{~s}$
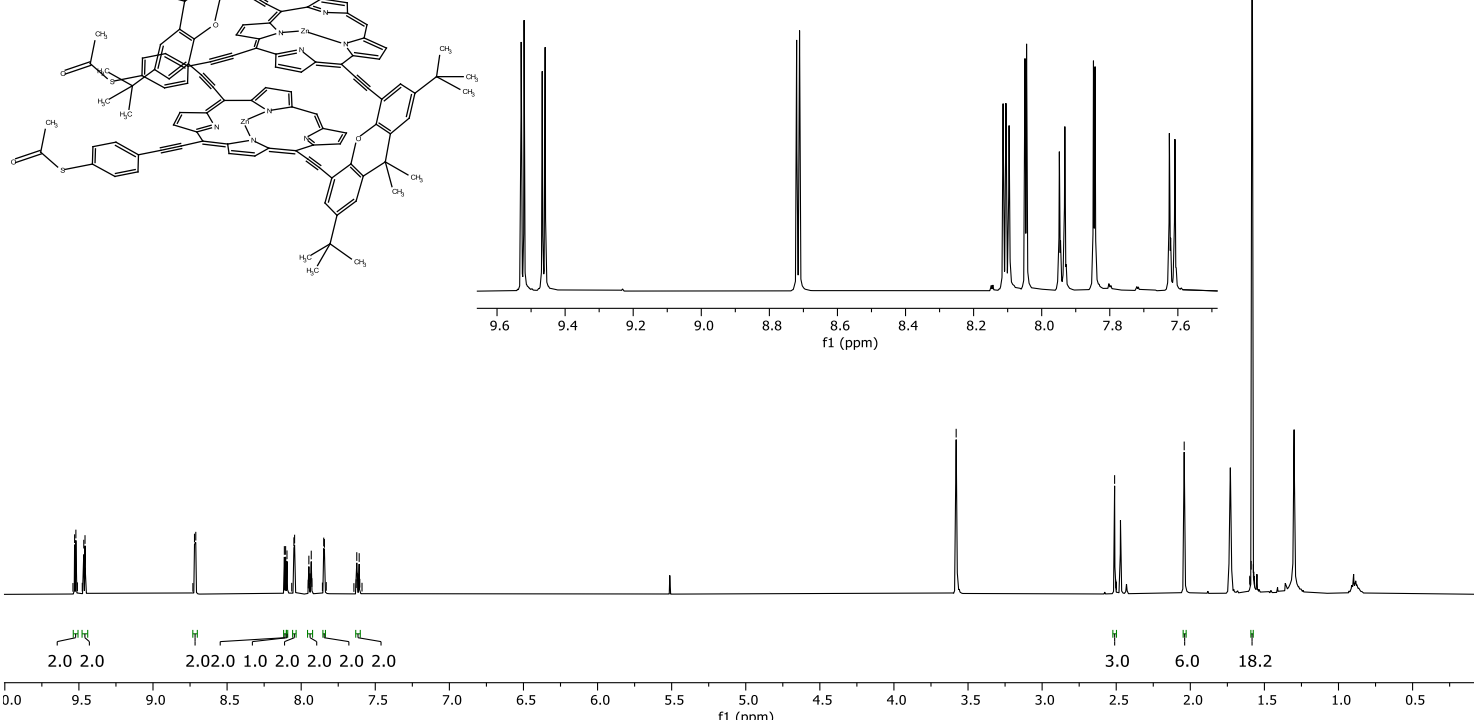

Figure S 50: ${ }^{1} \mathrm{H}-\mathrm{NMR}$ spectrum of 2.

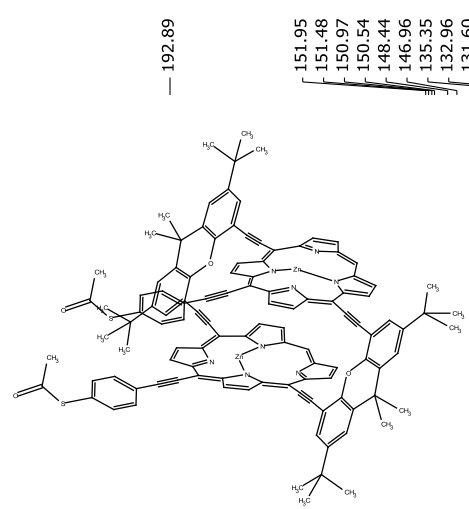

3C NMR (126 MHz, THE) 192.89, 151.95, 151.48, 150.97 ,

$150.54,148.44,146.96,135.35,132.96,131.60,131.40,131.32$,

$102.15,99.94,97,99,95.52,95.15,92,48,36.25,35,63,33.38$

$33.33,32.11,30.81$

监

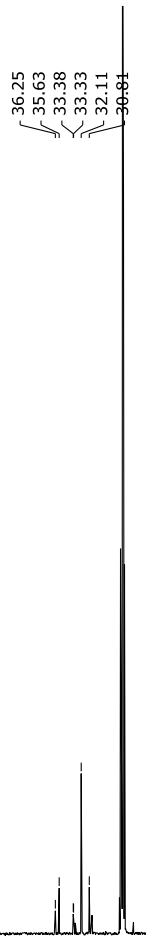

$\begin{array}{llllll}1 & 100 & 190 & 180 & 170 & 160\end{array}$

150

${ }_{130}^{1}+120$

100
$\mathrm{f} 1(\mathrm{ppm})$

Figure $S 51:{ }^{13} \mathbf{C}\left\{{ }^{1} \mathrm{H}\right\}-N M R$ spectrum of 2. 


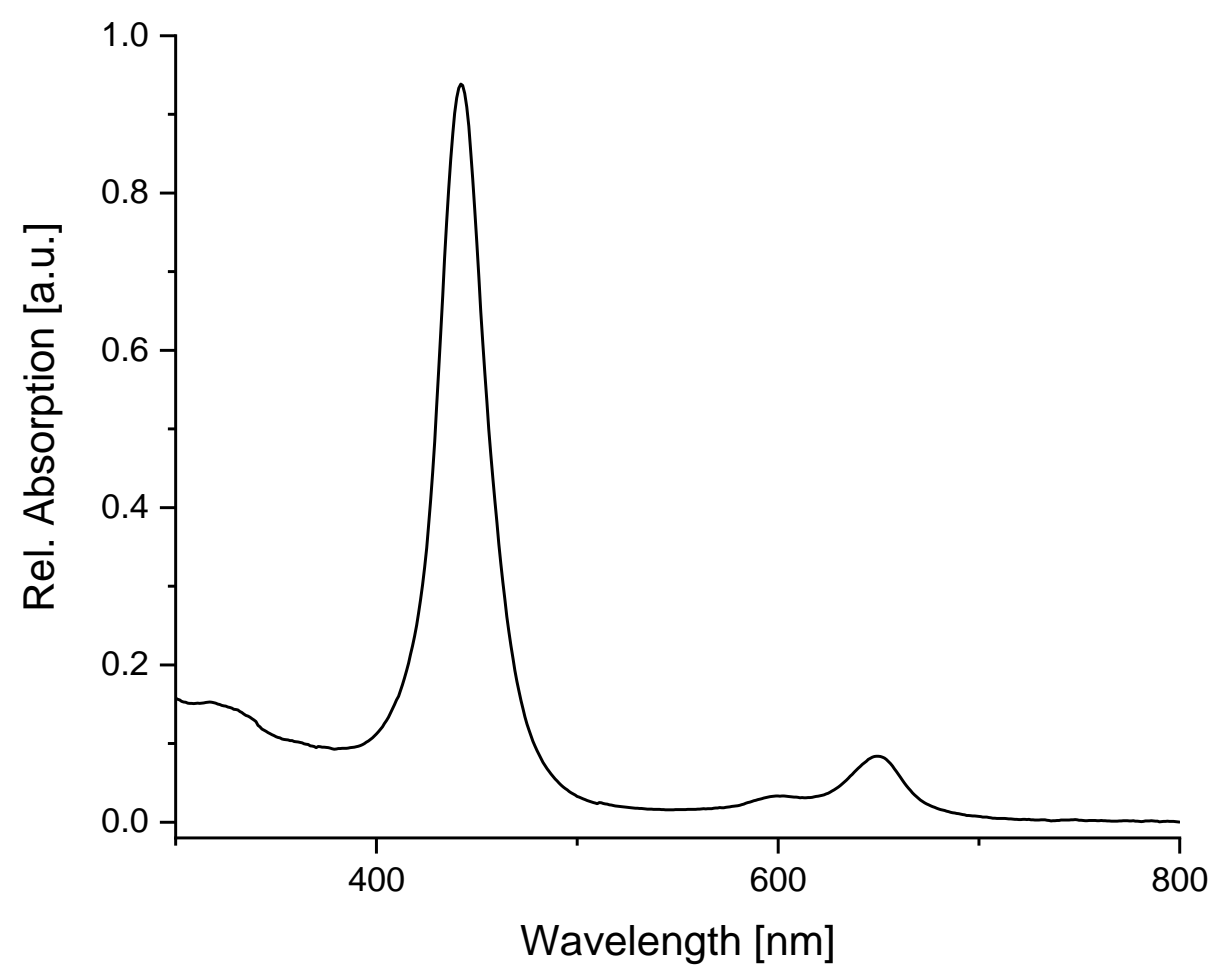

Figure $S$ 52: Absorption spectrum of 2 measured in $\mathrm{CH}_{2} \mathrm{Cl}_{2}$. 


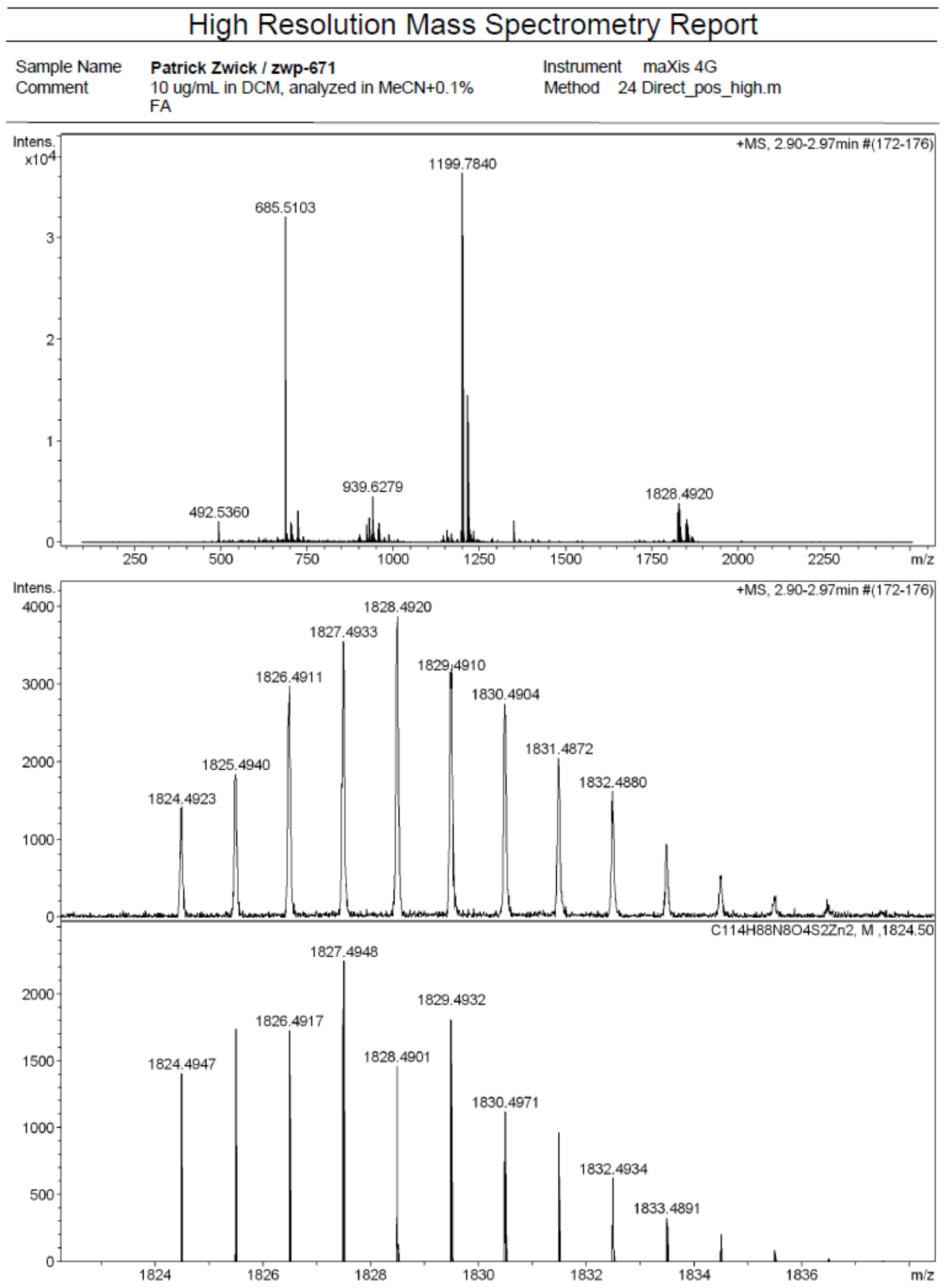

Figure S 53: HR-ESI MS of 2. 


\section{Method}

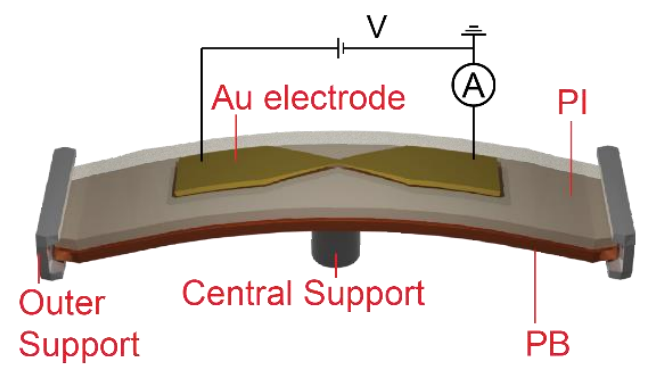

Figure S 54: MCBJ Schematics

Mechanically-controlled break junction. All the single-molecule measurements were performed on a homemade mechanically-controlled break junction (MCBJ) setup with nano-fabricated MCBJ flexible samples. A schematic of the MCBJ sample is shown in Fig. S54. A MCBJ sample consists of a flexible Phosphor bronze (PB) substrate, where an insulating layer of polyimide $(\mathrm{PI})$ is deposited. On top of the electrical insulation, 80 $\mathrm{nm}$ of gold electrodes are electron-beam patterned and evaporated with $5 \mathrm{~nm}$ Titanium adhesive layer. In a typical MCBJ fast-breaking measurement, the junction is opened and closed by a piezo-electric element pushing at the central support of the junction, with sub-nanometre resolution. During this opening and closing cycles, electrical conductance of the junction can be characterized by monitoring the current across the junction.

In the measurement described in the main text, the junction was first characterized without the molecule for the reference measurement. Afterward, a $5 \mu \mathrm{M}$ solution of 1 in $\mathrm{CH}_{2} \mathrm{Cl}_{2}$ was drop-casted onto the sample surface. The experiments were performed at dark in ambient condition. Fast-breaking measurements were performed by applying a constant DC bias voltage of $100 \mathrm{mV}$ and a constant electrode displacement speed at $2.5 \mathrm{~nm} / \mathrm{s}$. The junction conductance was recorded by monitoring the current across the junction with a homemade (TU Delft) logarithmic current amplifier. The technical details can be found in [1]. Self-breaking measurements were performed at constant applied bias of $100 \mathrm{mV}$ for the time frame of 120 seconds. Same as in the fast-breaking measurements, the current was constantly monitored by a logarithmic current amplifier to obtain the conductance values. The piezo-electric element was kept fixed at a constant displacement at the conductance of $4 \mathrm{G}_{0}$ for the self-breaking process. Once the junction conductance 
dropped to lower than $1 \mathrm{G}_{0}$ due to the self-breaking of the gold electrodes, the conductance was recorded for 120 seconds.

\section{References}

[1] Martin, C.A., Smit, R.H.M., Egmond, R. van, van der Zant, H.S.J., und van Ruitenbeek, J.M. (2011) A versatile low-temperature setup for the electrical characterization of single-molecule junctions. Review of Scientific Instruments, 82 (5), 053907. 